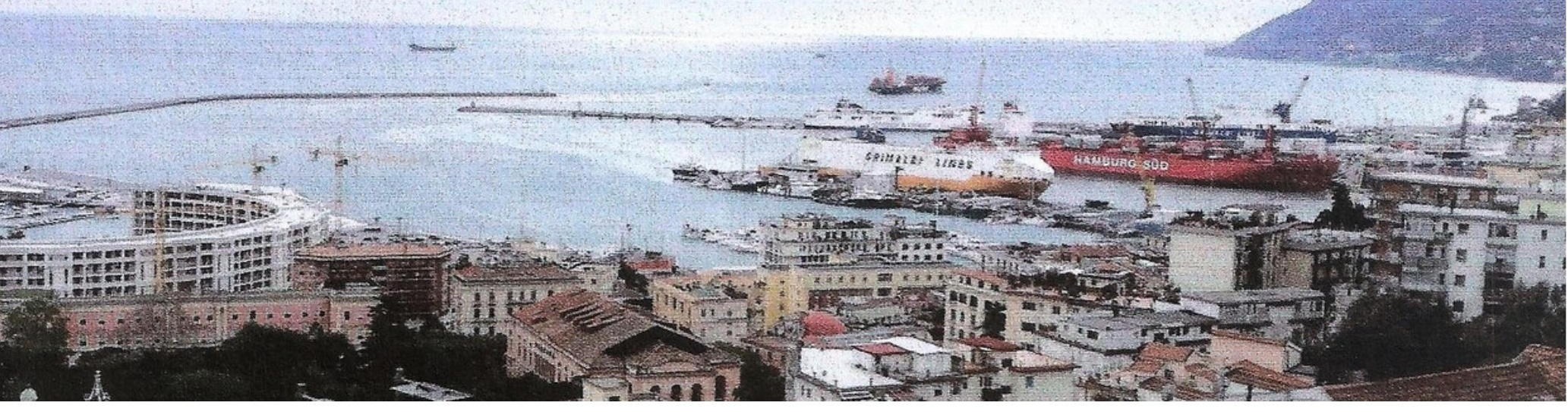

\title{
Breve Introduzione all'Improvvisazione Be-Bop [Short Introduction to Be-Bop Improvising]
}

Authors:
Submitted:
Published:
Volume:
Issue:
Affiliation:

Carmine Cataldo

30. July 2018

2. August 2018

5

6

Independent Researcher, PhD in Mechanical Engineering, MD in Disciplines of Jazz and Improvisation (Jazz Piano), Battipaglia (SA), Italy

Languages: Italian

Keywords: Jazz Improvisation, Be-Bop Language, Method, Triads, Seventh Chords, Scales, Substitutions

DOI: $\quad$ 10.17160/josha.5.6.448

Abstract: $\quad$ This paper represents a summary of a research work, carried out during the last two years, finalized to creating a method that may concretely assist jazz students in mastering the Be-Bop language. We have three main sections. The first section is predominantly devoted to the so-called linear language. We start from the mere definition of the fundamental Be-Bop scales, discussing their metric characteristics. Then, we introduce the notions of chromatism and approach, and suggest a strategy that may allow to easily combine the scales with the seventh arpeggios in the

\section{JOSHA Jumna orsemene. Humanities and Arts}




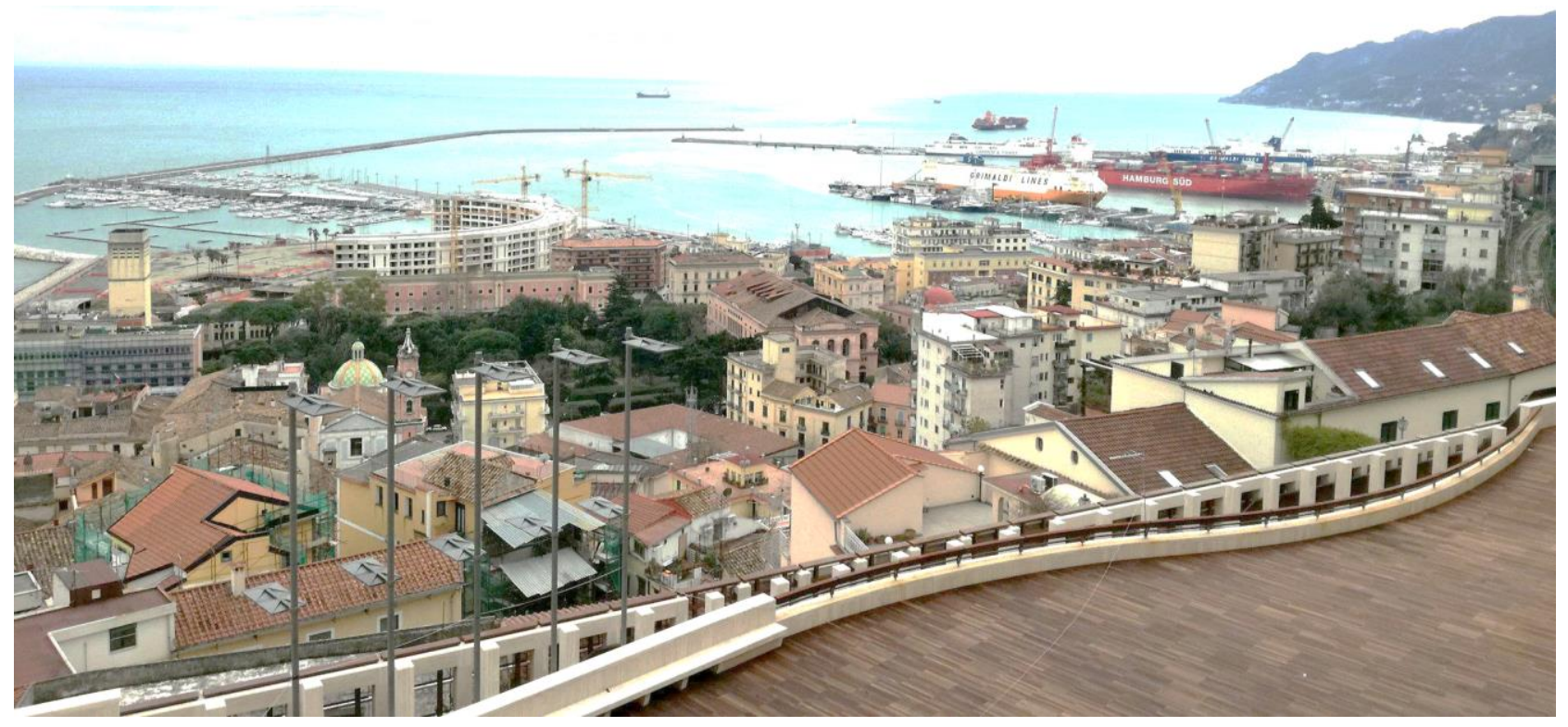

Vista del porto di Salerno (dalla finestra dell'aula 16bis del Conservatorio "G. Martucci”) 
A Mio Padre

Antonio Cataldo

improvvisamente scomparso

l'undici di Giugno dell'anno 2016

"[...] And you, my father, there on the sad height, curse, bless me now with your fierce tears, I pray.

Do not go gentle into that good night. Rage, rage against the dying of the light."

D. Thomas 
Prima Sezione

Il Linguaggio Lineare: le Scale Be-Bop

pag. 9

Introduzione alla Prima Sezione

pag. $\quad 11$

1. La Scala Dominante Be-Bop

pag. 13

1.1. Presentazione della Scala

pag. 13

1.2. Metabolizzare la Scala: Prime Frasi Elementari

pag. 13

1.3. Combinare Scale e Arpeggi

1.4. Osservazioni Finali

pag. 14

pag. 15

2. La Scala Minore Melodica Be-Bop

2.1. Presentazione della Scala

pag. 17

2.2. Metabolizzare la Scala: Prime Frasi Elementari

pag. 17

2.3. Combinare Scale e Arpeggi

pag. 17

pag. 18

2.4. Osservazioni Finali

pag. 19

3. La Scala Dorica Be-Bop

3.1. Presentazione della Scala

pag. $\quad 21$

pag. 21

3.2. Metabolizzare la Scala: Prime Frasi Elementari

pag. 21

3.3. Combinare Scale e Arpeggi

pag. 22

3.4. Osservazioni Finali

pag. 23

4. La Scala Minore Naturale Be-Bop

4.1. Presentazione della Scala

pag. $\quad 25$

pag. 25

pag. 25

pag. 26

4.3. Combinare Scale e Arpeggi

4.4. Osservazioni Finali

pag. $\quad 27$

5. La Scala Maggiore Be-Bop

pag. $\quad 29$

5.1. Presentazione della Scala

pag. 29

5.2. Metabolizzare la Scala: Prime Frasi Elementari

pag. 29

5.3. Combinare Scale e Arpeggi

pag. 31

5.4. Osservazioni Finali

pag. 32

\section{Seconda Sezione}

Il Linguaggio Articolato: Costruire sulle Triadi

pag. 35

Introduzione alla Seconda Sezione

pag. $\quad 37$

6. Improvvisare sugli Accordi di Prima Specie

pag. $\quad 39$

6.1. Presentazione della "Frase Fondamentale"

pag. 39

6.2. Focalizzare l'Attenzione sulla Triade Maggiore

pag. 39

6.3. Introduzione della Settima

pag. 41

6.4. Introduzione della Nona

pag. 43

6.5. Come "Arricchire" una Frase (cenni brevissimi)

pag. 43

6.6. Osservazioni Finali

pag. 44 
7. Improvvisare sugli Accordi di Seconda Specie

pag. $\quad 45$

7.1. Presentazione della "Frase Fondamentale"

pag. 45

7.2. Focalizzare l'Attenzione sulla Triade Minore

pag. 45

7.3. Ponti Cromatici e Terze Diatoniche

pag. 47

7.4. Tonicizzazione

7.5. Accordi "Parenti"

7.5.1. Accordo "Parente" di Prima Specie

pag. 49

pag. 49

pag. 49

7.5.2. Accordo "Parente" di Terza Specie

7.6. Osservazioni Finali

pag. $\quad 50$

pag. 51

8. Improvvisare sugli Accordi di Quarta Specie

pag. 53

8.1. Presentazione della "Frase Fondamentale"

pag. 53

8.2. Focalizzare l'Attenzione sulla Triade Maggiore

pag. 53

8.3. Introduzione della Settima

pag. 55

8.4. Introduzione della Nona

pag. 56

8.5. Tonicizzazione e Turnaround

pag. $\quad 57$

8.6. Osservazioni Finali

pag. 58

\section{Terza Sezione}

\section{Comprendere un Brano: Cenni all'Analisi Armonica}

pag. 59

Introduzione alla Terza Sezione

pag. $\quad 61$

9. Sostituzioni Armoniche Fondamentali

9.1. Sostituzioni Diatoniche

9.2. Sostituzioni per Dominanti Secondarie

pag. 63

9.3. Sostituzioni di Tritono

pag. 63

pag. 64

pag. 65

9.4. Sostituzioni per Espansione (Completamento) pag. 65

9.5. Sostituzioni alla Quinta Specie

pag. 66

10. Analisi di Alcuni Casi Notevoli

pag. $\quad 67$

10.1. Rhythm Changes (Bridge)

pag. 67

10.2. I'm Getting Sentimental Over You (prime 8 battute)

10.3. Stella by Starlight (prime 8 battute)

pag. $\quad 67$

pag. 68

10.4. Easy Living (prime 8 battute)

pag. 69

10.5. Giant Steps

pag. $\quad 70$

10.6. Osservazioni Finali

pag. 72

\section{Ringraziamenti}

pag. $\quad 73$

Bibliografia

pag. 75 


\section{Introduzione Generale e Sintesi del Lavoro}

Dando per scontata la conoscenza dei rudimenti storici relativi alla nascita del Be-Bop, ci si potrebbe anzitutto domandare che senso possa mai avere, oggi, intraprendere lo studio d'un linguaggio la cui creazione risale addirittura alla prima metà del secolo scorso.

Tanto per iniziare, potremmo affermare come il Be-Bop stia al jazz, nell'accezione più vasta del termine, come Bach alla musica classica (con accezione altrettanto vasta).

E come un musicista classico, degno d'essere definito tale, non dovrebbe nemmeno pensare di poter prescindere dalla conoscenza dell'Arte della Fuga e del Clavicembalo Ben Temperato, tanto per andarci giù pesante, così un jazzista che si rispetti non dovrebbe fare a meno d'affrontare uno studio, pure sufficientemente approfondito, di quello stile improvvisativo che, a cavallo degli anni ' 40 , fu concepito e formalizzato da mostri sacri come Parker, Gillespie e Powell.

Naturalmente, il volersi affrancare dall'idioma Be-Bop, dopo averne sufficientemente compreso il meccanismo, è cosa comprensibilissima nonché, dal punto di vista storico, addirittura auspicabile: al di là d'ogni ragionevole dubbio, infatti, se tutti avessero continuato a suonare come negli anni '40, non ci sarebbe stata alcuna evoluzione del linguaggio.

Essere jazzisti senza suonare Be-Bop, dunque, è assolutamente possibile: ma solo dopo aver imparato a suonare il Be-Bop! [al netto d'eccezioni tanto rare quanto, in certo senso, discutibili]

Tanto per fornire un esempio notevole, quando il "futuristico" Zawinul, nel '71, fondò i "Weather Report", poteva vantare circa un decennio di collaborazione con Cannonball (era lui il raffinatissimo pianista che accompagnò i fratelli Adderley per ottima parte degli anni '60).

E davvero sterile sarebbe speculare sul definire o meno "autenticamente Bop" il linguaggio adoperato da quello straordinario musicista viennese: lui il Be-Bop lo conosceva e lo suonava. Benissimo.

Il presente elaborato, dal carattere dichiaratamente introduttivo, scaturisce da un lavoro di ricerca, condotto negli ultimi due anni, concretizzatosi nella pubblicazione, a valle d'un doveroso processo di revisione paritaria, di numerosi articoli su riviste scientifiche di rilievo internazionale.

Si è optato per una suddivisione in tre sezioni.

La Prima Sezione è dedicata allo studio delle cosiddette scale Be-Bop, cui è inevitabilmente correlato lo sviluppo di un linguaggio decisamente lineare.

Un minimo di varietà, per così dire, è introdotta ricorrendo all'utilizzo parsimonioso, limitatamente alla sezione in oggetto, di cromatismi, note d'approccio ed "enclosure", ovvero adottando una semplice metodologia finalizzata alla fusione tra scale e arpeggi di settima.

Si dimostra, inoltre, come alcune delle scale presentate si prestino ad essere adoperate su intere progressioni armoniche, ottenendo una sonorità marcatamente Be-Bop tutt'altro che banale, deliberatamente ignorando la successione degli accordi. 
La Seconda Sezione è dedicata allo sviluppo del linguaggio articolato.

Il metodo è relativamente innovativo: al netto di alcune eccezioni, tra l'altro apparenti, l'attenzione dell'improvvisatore è mantenuta costantemente sulle triadi (in stato fondamentale ovvero rivoltate). In certo senso, è possibile stabilire un parallelismo tra le note cordali pure (quelle costituenti la triade) e i costituenti fondamentali di una proposizione semplice (soggetto, verbo e complemento).

Il sistema si fonda sostanzialmente sulla metabolizzazione di alcune "frasi fondamentali" (una per ogni tipologia di accordo), dall'opportuna modificazione delle quali (sostanzialmente, tagli e aggiunte di "parole" elementari) è possibile costruire un vocabolario realmente sconfinato, senz'alcun bisogno di possedere un background di carattere "boppistico".

Nella Terza Sezione, la più breve delle tre, si forniscono dei cenni all'analisi armonica di un brano. In breve, vengono descritte le principali sostituzioni armoniche (tenendo deliberatamente fuori quella per "scambio parallelo"), discutendone l'applicazione in riferimento ad alcuni casi notevoli.

I riferimenti discografici, selezionati per rilevanza storica e/o didattica, sono inseriti sul retro di alcune delle pagine del volume, con tanto di copertina (quella più nota, nel caso di cover multiple).

I riferimenti bibliografici sono apposti in maniera progressiva lungo l'intero lavoro, in tal modo evitando l'azzeramento della numerazione all'inizio di ogni capitolo. 


\section{Prima Sezione}

\section{Il Linguaggio Lineare: \\ le Scale Be-Bop}


I capitoli di questa sezione (cinque in tutto) sono dedicati alle cosiddette scale Be-Bop.

La struttura dei vari capitoli è intenzionalmente identica.

Il Primo Paragrafo è rivolto alla presentazione della particolare scala (intenzionalmente estesa lungo due ottave consecutive), evidenziandone le peculiarità metriche. In breve, trattiamo scale octofoniche la cui esecuzione colloca, in battere, alcune "note fondamentali". Le note di cui sopra coincidono con le cordali pure (le note costituenti la triade) cui si aggiunge, a seconda della scala, la sopradominante ovvero la sottotonica.

Il Secondo Paragrafo è indirizzato alle tecniche di metabolizzazione della particolare scala nonché alla creazione di prime frasi elementari. Viene suggerita l'esecuzione della scala, in moto discendente, partendo dalle "note fondamentali" ovvero, avvalendosi di artifizi tipici dell'idioma Be-Bop (quali cromatismi, note d'approccio ed "enclosure"), dalle tensioni residue.

Il Terzo Paragrafo è dedicato alla combinazione di frammenti di scala (ovvero frasi brevi costruite sulla scala) ed arpeggi di settima (quadriadi terziane diatoniche dedotte dall'armonizzazione della scala eptafonica corrispondente).

Il Quarto Paragrafo è riservato alle osservazioni finali, con attenzione particolare rivolta alle metodologie di studio degli esercizi proposti nonché ai campi d'applicazione delle frasi dedotte. 


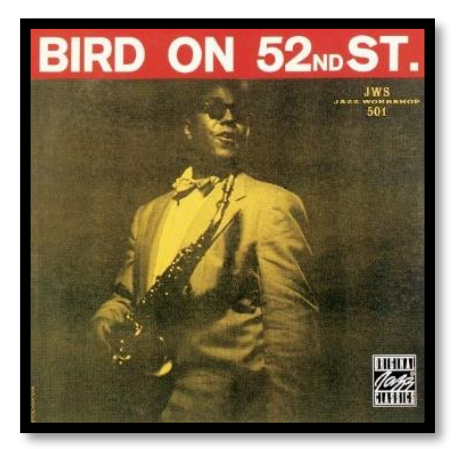

CHARLIE PARKER SWEDISH SCHNAPPS +

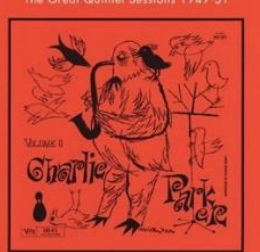

Miles Davis, Red Rodnoy, Kenny Dorham
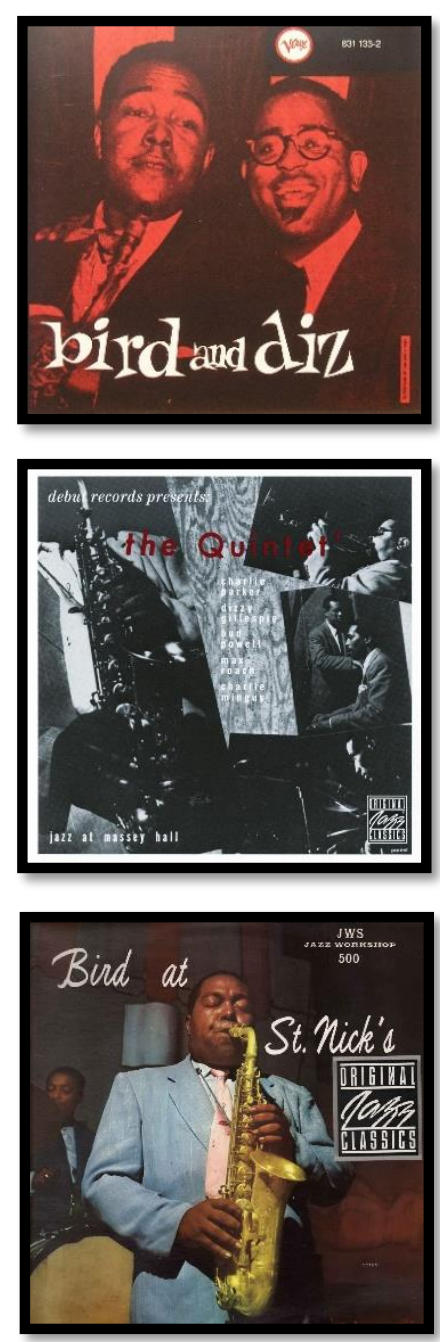

\section{Bird on 52nd Street}

(Debut, 1948)

Charlie Parker - sassofono contralto

Miles Davis - tromba

Duke Jordan - pianoforte

Tommy Potter - contrabbasso

Max Roach - batteria

\section{Swedish Schnapps}

(Verve, 1951)

Charlie Parker - sassofono contralto Red Rodney / Miles Davis - tromba John Lewis / Walter Bishop Jr. - pianoforte Ray Brown / Teddy Kotick - contrabbasso Kenny Clarke / Max Roach - batteria

\section{Bird and Diz \\ (Clef, 1952)}

Charlie Parker - sassofono contralto Dizzy Gillespie / Kenny Dorham - tromba Tommy Turk - trombone Thelonious Monk / Al Haig - pianoforte Curly Russell / Tommy Potter - contrabbasso Buddy Rich / Max Roach - batteria Carlos Vidal - bonghi

\section{Jazz at Massey Hall}

(Debut, 1953)

Charlie Parker - sassofono contralto Dizzy Gillespie - tromba Bud Powell - pianoforte Charles Mingus - contrabbasso Max Roach - batteria

\section{Bird at St. Nick's}

(JWS 500, 1957)

Charlie Parker - sassofono contralto

Red Rodney - tromba

$\mathrm{Al}$ Haig - pianoforte

Tommy Potter - contrabbasso

Roy Haynes - batteria 


\section{La Scala Dominante Be-Bop}

\subsection{Presentazione della Scala}

Si riporta di seguito la Scala Dominante Be-Bop di $C$ [1], nei moti ascendente e discendente, intenzionalmente estesa lungo due ottave consecutive.

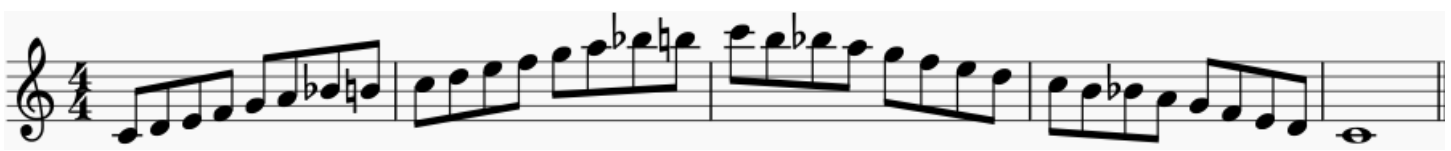

Tale scala trova immediata ed efficace applicazione in presenza dell'accordo $C 7$. [2]

L'aggiunta della sensibile è fondamentalmente correlata all'ottenimento di un'interessante quadratura metrica. Eseguendo la scala a partire dalle note cordali (quelle costituenti l'accordo di prima specie corrispondente), quest'ultime risulteranno sempre posizionate in battere. Logicamente, le tensioni (nona, undicesima, tredicesima) saranno sempre collocate in levare.

\subsection{Metabolizzare la Scala: Prime Frasi Elementari}

Uno dei primi esercizi in questa sede proposti, finalizzato ad una rapida metabolizzazione della scala, consiste nel suonare la Dominante Be-Bop in moto discendente, partendo da ognuna delle note cordali e raggiungendo, in ogni caso, la tonica (all'ottava inferiore rispetto a quella di partenza).

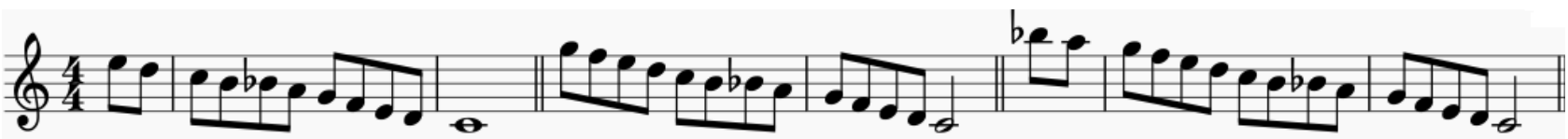

La frase con partenza dalla tonica è stata evidentemente omessa per brevità.

Per quanto affermato in precedenza, la quadratura metrica viene persa qualora la nota di partenza coincida con una delle tensioni. Il recupero metrico, tuttavia, è agevolmente ottenibile a mezzo di alcuni artifizi, assai diffusi nell'idioma Be-Bop. Il più banale consiste semplicemente nell'imporre un'anacrusi: in altri termini, la prima nota, che coincide con una delle tensioni, è suonata in levare. È inoltre possibile ribattere la tensione di partenza: coerentemente con la figurazione finora adoperata, pertanto, la frase inizierà, in tal caso, con due crome identiche consecutive. Alternativamente, è possibile raddoppiare la durata della tensione di partenza: sempre nel rispetto della figurazione adottata, la frase inizierà, in tal caso, con una semiminima. Inoltre, sono contemplabili soluzioni, decisamente più interessanti, basate su cromatismi ed approcci misti (alle note cordali).

Proponiamo di seguito tre semplicissime frasi con partenza dalla nona.

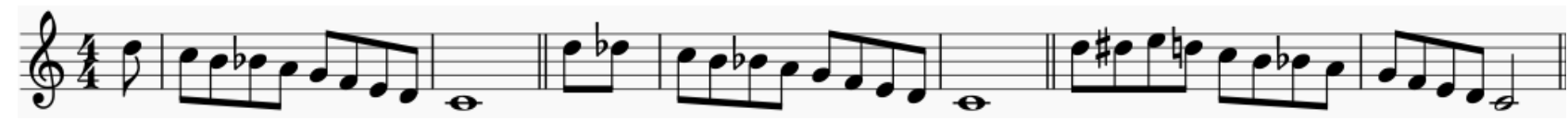


La prima frase è stata ottenuta con un'anacrusi, la seconda con un ponte cromatico (in anacrusi) verso la tonica, la terza costruendo un ponte cromatico verso la modale. [3] A scopo squisitamente esercitativo, le frasi proposte terminano sulla tonica (all'ottava inferiore rispetto a quella di partenza).

Proponiamo, adesso, tre semplicissime frasi con partenza dall'undicesima.

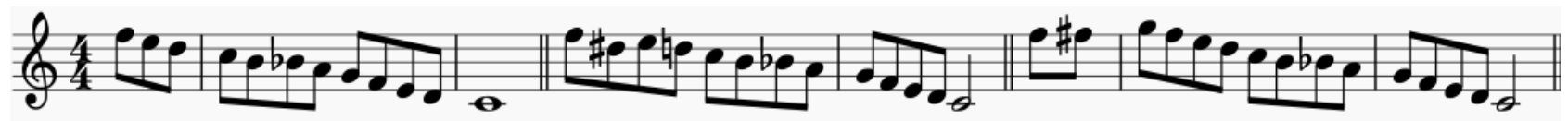

La prima frase è stata ottenuta imponendo un'anacrusi (la cui durata è pari a $3 / 8$ al solo fine di collocare la tonica sul primo movimento dell'ultima battuta), la seconda adoperando nulla più che un approccio misto alla modale (la sottodominante coincide con la nota d'approccio discendente alla modale), la terza costruendo un ponte cromatico (in anacrusi) verso la dominante. [3] Ancora una volta, le frasi terminano deliberatamente sulla tonica (all'ottava inferiore rispetto a quella di partenza).

Il secondo degli esempi sopra discussi ci offre l'opportunità di ricordare come, una volta definita la scala di riferimento, l'approccio alle note cordali pure (quelle costituenti la triade, essendo la settima soventemente qualificata come tensione fondamentale) è cromatico qualora ascendente, diatonico qualora discendente.

Proponiamo, infine, tre semplicissime frasi con partenza dalla tredicesima.

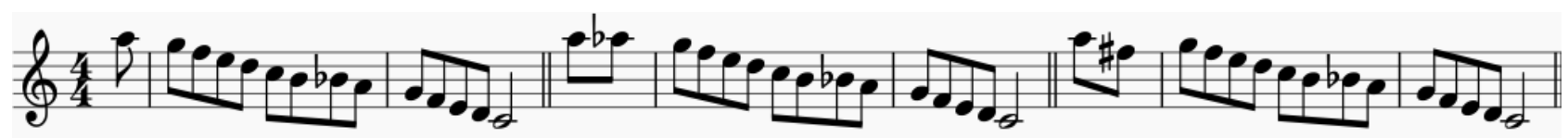

La prima frase è stata ottenuta imponendo un'anacrusi, la seconda costruendo (in anacrusi) un ponte cromatico verso la dominante, la terza effettuando (sempre in anacrusi) un approccio misto alla dominante (la sopradominante è nota d'approccio discendente alla dominante). [3] Nuovamente, tutte le frasi sono arrestate alla tonica (all'ottava inferiore).

\subsection{Combinare Scale e Arpeggi}

Altro utilissimo esercizio consiste nell'esecuzione delle quadriadi terziane diatoniche relative alla tonalità di pertinenza. Nel caso in questa sede proposto (stiamo ipotizzando d'improvvisare su C7), considereremo accordi di settima arpeggiati [4], in moto ascendente, dedotti dall'armonizzazione [5] [6] [7] della Ionica di $F$ ovvero, equivalentemente, della Misolidia di $C$.

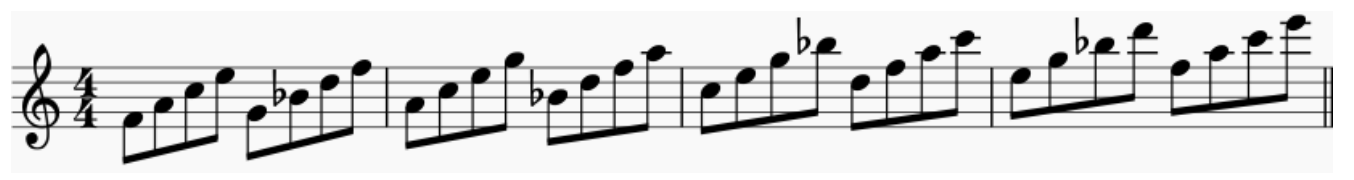

Le quadriadi terziane diatoniche possono essere agevolmente combinate con le frasi costruite sulla Dominante Be-Bop: molto semplicemente, l'ultima nota dell'arpeggio rappresenterà l'elemento di congiunzione tra il suddetto e la scala. A titolo d'esempio, la quarta nota dell'arpeggio ascendente di Fmaj7 è $E$ (simultaneamente sensibile rispetto a $F$ e modale rispetto a $C$ ): pertanto, il suddetto arpeggio può agganciarsi ad una qualsivoglia frase, costruita sulla Dominante Be-Bop, che preveda partenza dalla terza. Assai spesso, inoltre, si preferisce approcciare ascendentemente (e quindi, per definizione, cromaticamente) la prima nota dell'arpeggio. 
Proponiamo, di seguito, alcuni esempi (uno per ognuna delle note cordali di $C 7$, in corrispondenza delle quali ha luogo la giunzione) finalizzati ad illustrare qualitativamente il modo in cui le quadriadi terziane diatoniche possano efficacemente combinarsi con la Dominante Be-Bop.
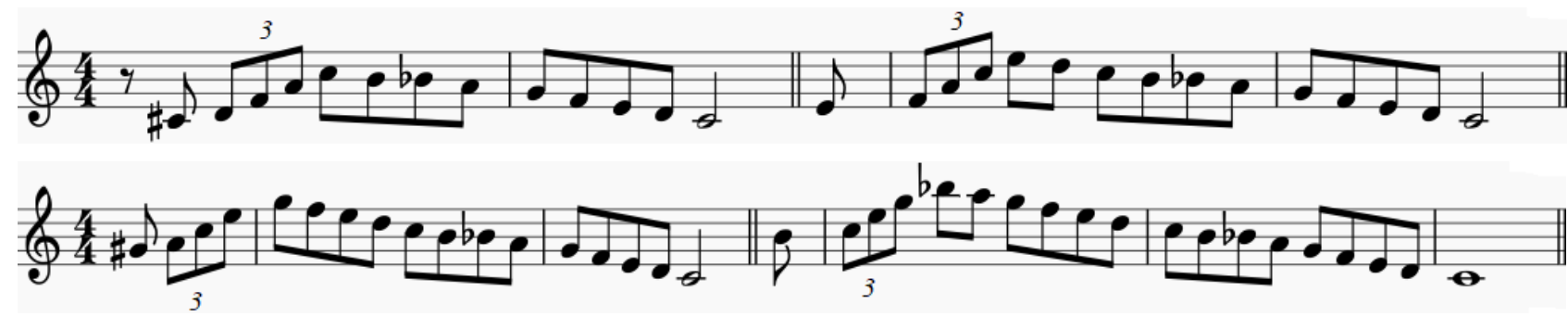

La prima nota dell'arpeggio è sempre approcciata. Le prime tre note costituiscono una terzina: l'adozione di tale gruppo irregolare, sebbene tutto fuorché strettamente necessaria, costituisce elemento distintivo dell'idioma Be-Bop. Come sempre in questa sede, le frasi proposte terminano sulla tonica (all'ottava inferiore rispetto a quella di partenza).

Proponiamo alcuni esempi più articolati costruiti sulle tensioni (due per ognuno dei gradi tensivi).
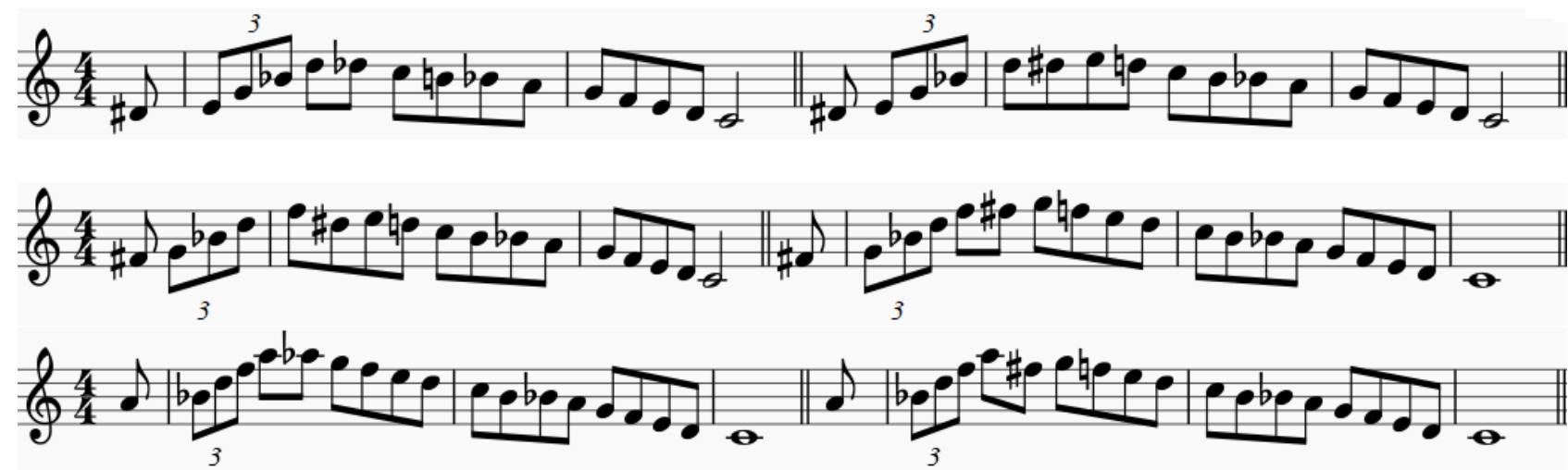

\subsection{Osservazioni Finali}

Sarebbe fin da subito opportuno affrontare lo studio nelle dodici tonalità procedendo, ad esempio, per quarte giuste ascendenti (ovvero, equivalentemente, per quinte giuste discendenti). Una prima fase dello studio potrebbe consistere nella mera esecuzione della scala; la seconda fase potrebbe essere dedicata alla riproduzione della scala partendo da ognuna delle note cordali; nella terza, magari, si potrebbe rivolgere l'attenzione alle tensioni, e così via. In ognuna delle fasi, naturalmente, lo studio andrebbe condotto nelle dodici tonalità. Si precisa come l'adozione della suddetta metodologia sia da considerarsi implicitamente suggerita per ognuno dei capitoli appartenenti alla sezione corrente.

Sebbene l'argomento non sia, in questa sede, oggetto di discussione, è doveroso sottolineare come la scala Dominante Be-Bop (adoperata tal quale ovvero come elemento costitutivo di frasi più complesse) possa essere impiegata anche in presenza di accordi di seconda e terza specie. In altri termini, molte delle frasi finora ricavate per $C 7$ si rivelano compatibili con $G m i n 7$ e Emin $7 b 5$. D'altra parte, sarà successivamente evidenziato (si veda il Capitolo 7) come, sotto alcune condizioni, le frasi idonee a Emin $7 b 5$ siano compatibili con $C 7$ e $G \min 7$, essendo gli ultimi due accordi ricavabili dal precedente per sostituzione diatonica [7] [8] [9] (Gmin7 e $C 7$ sono pertanto vincolati da una parentela, per così dire, di secondo grado, formalizzabile attraverso la sostituzione per espansione). [8] [9] 

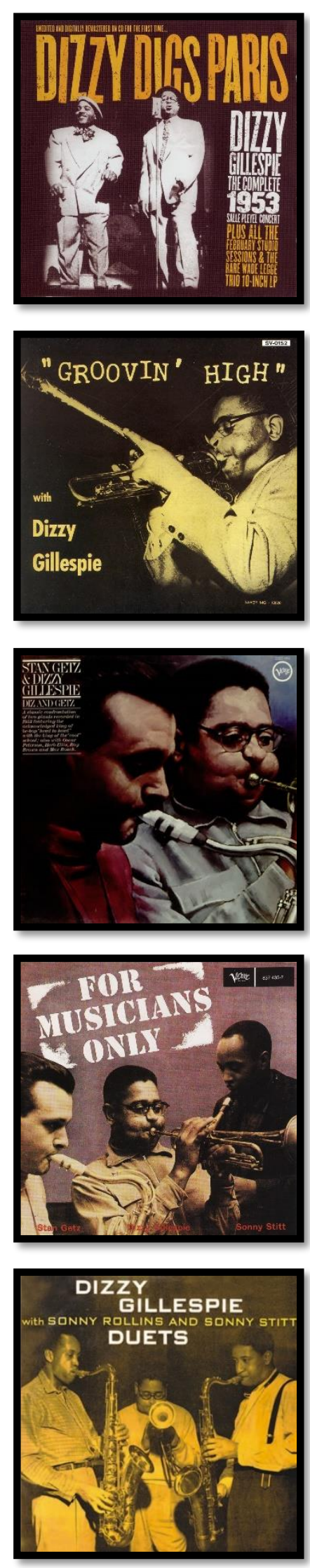

\section{Dizzy Digs Paris}

(Disques Vogue, 1953 - Giant Steps, 2006)

Dizzy Gillespie - tromba, pianoforte, voce, congas

Bill Graham - sassofoni contralto e baritono

Nat Peck - trombone

Wade Legge - pianoforte

Lou Hackney - contrabbasso

Al Jones - batteria

Joe Carroll / Sarah Vaughan - voce

The Paris Operatic String Orchestra

\section{Groovin' High}

(Savoy, 1955)

Dizzie Gillespie / Kenny Dorham et al. - tromba

Charlie Parker / Sonny Stitt et al. - sassofono contralto

Dexter Gordon / Lucky Thompson et al. - sassofono tenore

Alton Moore / Gordon Thomas et al. - trombone

Remo Palmieri / Chuck Wayne - chitarra

Milt Jackson - vibrafono

Al Haig / Clyde Hart et al. - pianoforte

Curly Russel / Slam Stewart et al. - contrabbasso

Kenny Clarke / Cozy Cole et al. - batteria

\section{Diz and Getz}

(PolyGram, 1955)

Dizzie Gillespie - tromba, voce

Stan Getz / Hank Mobley - sassofono tenore Herb Ellis - chitarra

Oscar Peterson / Wade Legge - pianoforte

Ray Brown / Lou Hackney - contrabbasso

Max Roach / Charlie Persip - batteria

\section{For Musicians Only}

(Verve, 1958)

Dizzy Gillespie - tromba

Sonny Stitt - sassofono contralto

Stan Getz - sassofono tenore

Herb Ellis - chitarra

John Lewis - pianoforte

Ray Brown - contrabbasso

Stan Levey - batteria

\section{Duets}

(Verve, 1958)

Dizzy Gillespie - tromba

Sonny Stitt - sassofoni contralto e tenore

Sonny Rollins - sassofono tenore

Ray Bryant - pianoforte

Tommy Bryant - contrabbasso

Charlie Persip - batteria 


\section{La Scala Minore Melodica Be-Bop}

\subsection{Presentazione della Scala}

Si riporta di seguito la Scala Minore Melodica Be-Bop di $C$ [10], nei moti ascendente e discendente, estesa lungo due ottave consecutive.

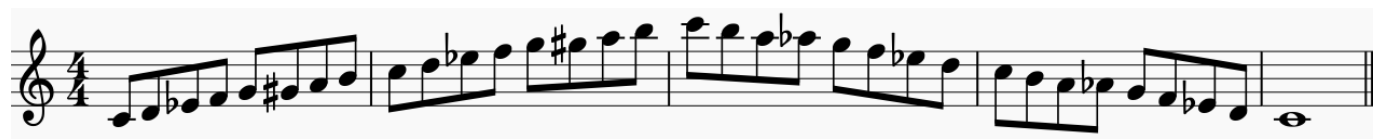

A rigore, la scala appena presentata trova applicazione in presenza dell'accordo $C-6$ [2]: tuttavia, se ne riscontra ampio utilizzo anche sull' accordo $C-\Delta$ (sesta specie) e addirittura, sebbene in modo formalmente illegittimo, su $C-7$ (seconda specie).

L'aggiunta della sesta minore (rispetto alla Minore Melodica ascendente ovvero all'Ipoionica) è correlata all'ottenimento di un'interessante quadratura metrica. Eseguendo la scala a partire dalle note cordali pure (tonica, modale, dominante) e dalla sesta (sopradominante), queste risulteranno sempre posizionate in battere. Le tensioni residue (settima, nona, undicesima,) saranno collocate in levare.

\subsection{Metabolizzare la Scala: Prime Frasi Elementari}

Uno dei primi esercizi in questa sede proposti, finalizzato ad una rapida metabolizzazione della scala, consiste nel suonare la Minore Melodica Be-Bop in moto discendente, partendo dalle note cordali pure e dalla sesta (maggiore), raggiungendo, in ogni caso, la tonica (all'ottava inferiore rispetto a quella di partenza).

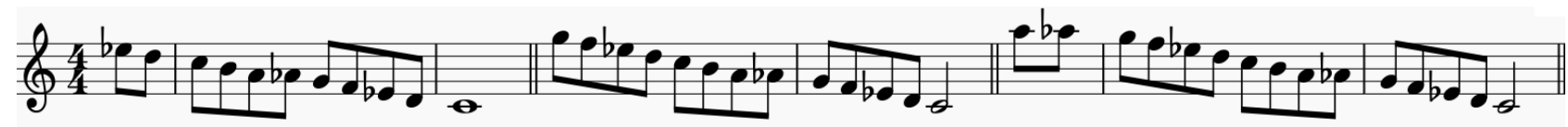

La frase con partenza dalla tonica è stata evidentemente omessa per brevità.

Per quanto affermato in precedenza, la quadratura metrica viene persa qualora la nota di partenza coincida con una delle tensioni residue (la settima, considerata in questa sede quale tensione fondamentale, la nona, l'undicesima). Il recupero metrico, tuttavia, è agevolmente ottenibile a mezzo di alcuni artifizi, assai diffusi nell'idioma Be-Bop. Il più banale consiste semplicemente nell'imporre un'anacrusi: in pratica, la prima nota, che coincide con una delle tensioni residue, è suonata in levare. È inoltre possibile ribattere la tensione di partenza: coerentemente con la figurazione finora adoperata, pertanto, la frase inizierà, in tal caso, con due crome identiche consecutive. Alternativamente, è possibile raddoppiare la durata della tensione di partenza: sempre nel rispetto della figurazione adottata, la frase inizierà, in tal caso, con una semiminima. Inoltre, sono contemplabili soluzioni, talvolta decisamente più interessanti, basate su cromatismi ed approcci misti (alle note cordali pure).

Proponiamo di seguito tre semplicissime frasi con partenza dalla sopratonica (ovvero dalla nona). 


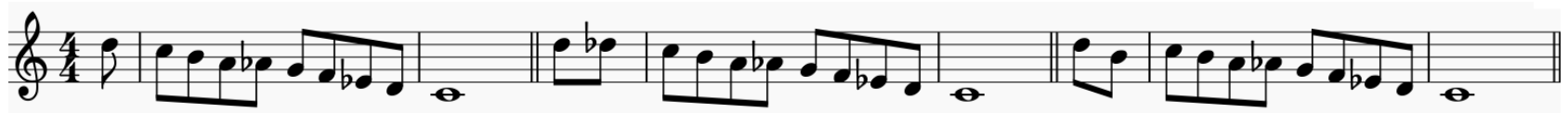

La prima è frase stata ottenuta imponendo un'anacrusi, la seconda costruendo (in anacrusi) un ponte cromatico verso la tonica, la terza adoperando (sempre in anacrusi) un approccio misto alla tonica. [3] A scopo squisitamente esercitativo, tutte le frasi proposte terminano sulla tonica (all'ottava inferiore rispetto a quella di partenza).

L'ultimo degli esempi sopra discussi ci offre l'opportunità di ricordare che, una volta definita la scala di riferimento, l'approccio alle note cordali pure (quelle costituenti la triade, essendo la settima soventemente ritenuta, come già sottolineato in precedenza, tensione fondamentale) è cromatico qualora ascendente, diatonico qualora discendente.

Proponiamo, adesso, tre semplicissime frasi con partenza dalla sottodominante (dall'undicesima).

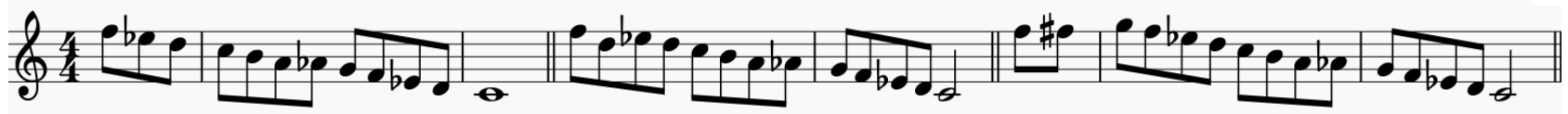

La prima frase è stata ottenuta imponendo un'anacrusi (la cui durata è pari a $3 / 8$ al solo fine di collocare la tonica sul primo movimento dell'ultima battuta), la seconda adoperando nulla più che un approccio misto alla modale (la sottodominante coincide con la nota d'approccio discendente alla modale), la terza costruendo (in anacrusi) un ponte cromatico verso la dominante. [3] Ancora una volta, le frasi terminano deliberatamente sulla tonica (all'ottava inferiore rispetto a quella di partenza).

Proponiamo, infine, tre semplicissime frasi con partenza dalla sensibile (settima maggiore).

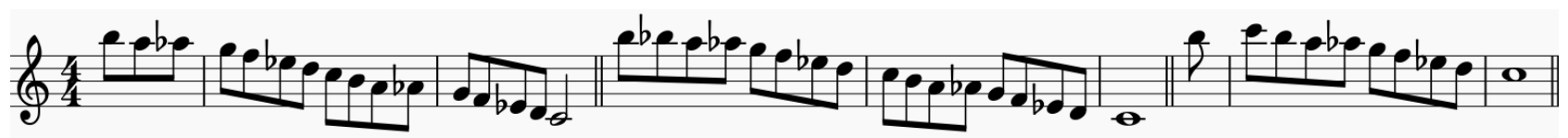

La prima frase è stata ottenuta imponendo un'anacrusi (la cui durata è pari a $3 / 8$ al solo fine di collocare la tonica sul terzo movimento dell'ultima battuta), la seconda costruendo un ponte cromatico verso la sopradominante (e quindi, considerata la struttura della scala, verso la dominante), la terza effettuando (in anacrusi) un approccio cromatico ascendente alla tonica (all'ottava superiore). [3] Nuovamente, tutte le frasi sono intenzionalmente arrestate alla tonica.

\subsection{Combinare Scale e Arpeggi}

Altro utilissimo esercizio consiste nell'esecuzione delle quadriadi terziane diatoniche deducibili dalla Minore Melodica (ascendente) ovvero, equivalentemente, dall'Ipoionica (Bachiana). Nel caso in questa sede proposto (stiamo ipotizzando d'improvvisare, formalmente, su $C-6$ ovvero $C-\Delta$ ), considereremo accordi di settima arpeggiati [4], in moto ascendente, dedotti dall'armonizzazione [5] [6] [7] della Minore Melodica (ascendente) di $C$.

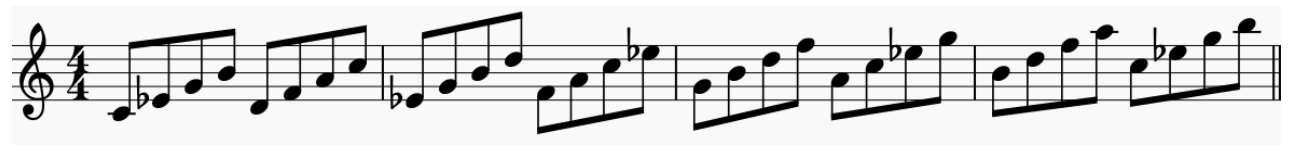

Le quadriadi terziane diatoniche possono essere agevolmente combinate con le frasi costruite sulla Minore Melodica Be-Bop: molto semplicemente, l'ultima nota dell'arpeggio rappresenterà 
l'elemento di congiunzione tra il suddetto e la scala. A titolo d'esempio, la quarta nota dell'arpeggio ascendente di $F 7$ è $E^{b}$ (simultaneamente sottotonica rispetto ad $F$ e modale rispetto a $C$ ): pertanto, tale arpeggio potrà agganciarsi ad una qualsivoglia frase, costruita sulla Minore Melodica Be-Bop, che preveda partenza dalla terza (minore). Assai spesso, inoltre, si inserisce un approccio ascendente (e quindi, per definizione, cromatico) alla prima nota dell'arpeggio.

Proponiamo, di seguito, alcuni esempi (uno per ognuna delle note cordali pure, costituenti la triade minore di $C$, ed uno per la sopradominante) al fine d'illustrare qualitativamente il modo in cui le quadriadi terziane diatoniche possano combinarsi con la scala Minore Melodica Be-Bop.
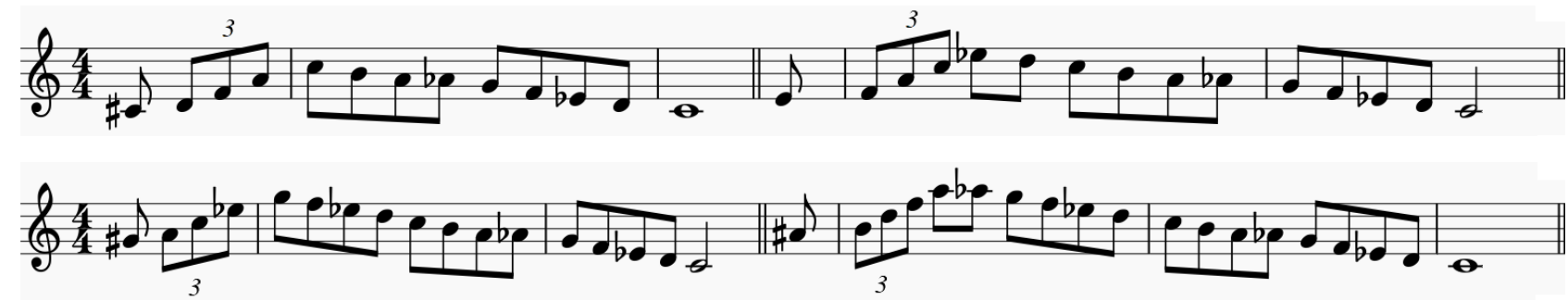

La prima nota dell'arpeggio è sempre approcciata. Le prime tre note costituiscono una terzina: l'adozione di tale gruppo irregolare, sebbene tutto fuorché strettamente necessaria, costituisce elemento distintivo dell'idioma Be-Bop. [3] Come sempre in questa sede, le frasi proposte terminano sulla tonica (all'ottava inferiore rispetto a quella di partenza).

Proponiamo alcuni esempi più articolati sulle tensioni residue (due frasi per ognuno dei gradi tensivi).
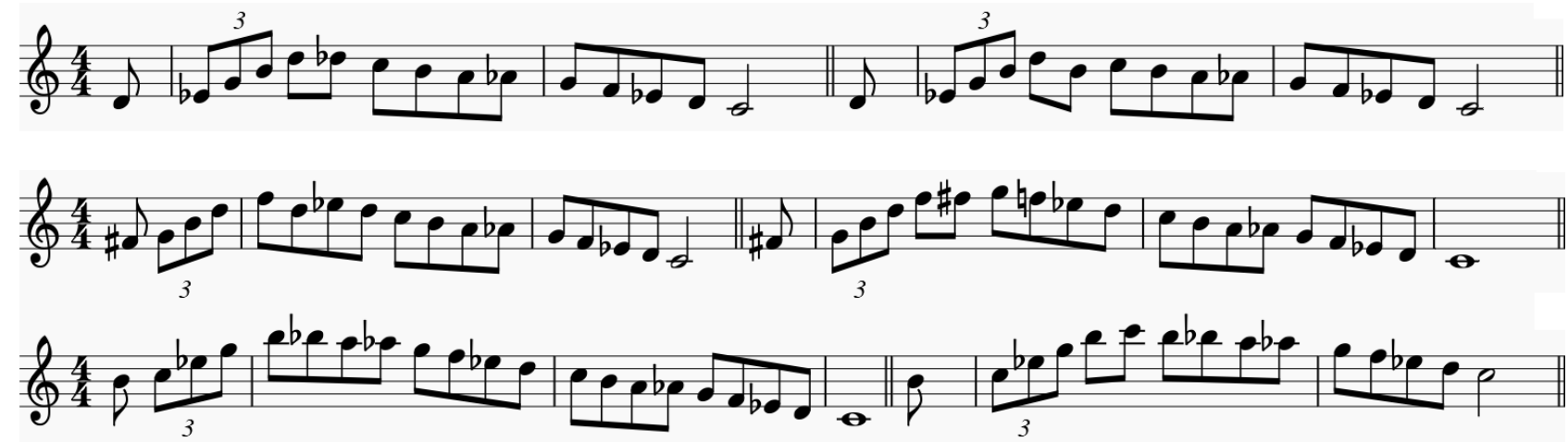

\subsection{Osservazioni Finali}

La riproduzione degli esempi proposti contribuisce indubbiamente all'acquisizione di una notevole padronanza della scala Minore Melodica Be-Bop, e in ciò risiede la principale finalità del lavoro. Tuttavia, è d'uopo sottolineare come la pedissequa esecuzione di alcune delle frasi presentate possa non risultare del tutto efficace qualora in presenza di un solo accordo $(C-6$ ovvero, al limite, $C-\Delta$, per attenerci al caso considerato in questa sede): nondimeno, il problema è agevolmente risolvibile imponendo, in corrispondenza delle anacrusi, la presenza dell'accordo tonicizzante [8] [9] (G7, sempre a volersi attenere al caso particolare trattato nel presente articolo), ancor meglio se opportunamente alterato. 

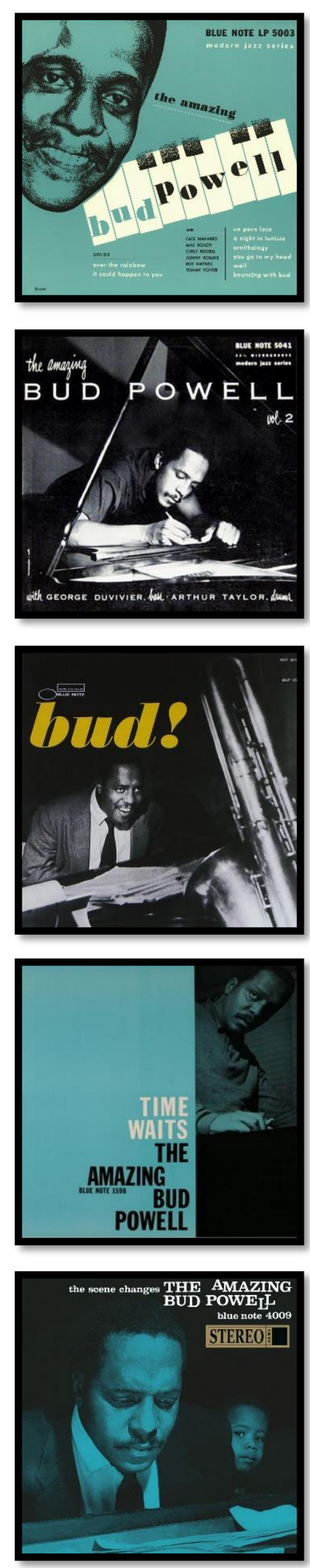

The Amazing Bud Powell vol. 1

(Blue Note, 1952)

Fats Navarro - tromba

Sonny Rollins - sassofono tenore

Bud Powell - pianoforte

Tommy Potter / Curly Russell - contrabbasso

Roy Haynes / Max Roach - batteria

\section{The Amazing Bud Powell vol. 2}

(Blue Note, 1954)

Bud Powell - pianoforte

George Duvivier / Tommy Potter - contrabbasso

Art Taylor / Roy Haynes - batteria

\section{Bud! (The Amazing Bud Powell vol. 3)}

(Blue Note, 1957)

Bud Powell - pianoforte

Curtis Fuller - trombone

Paul Chambers - contrabbasso

Art Taylor - batteria

\section{Time Waits (The Amazing Bud Powell vol. 4)}

(Blue Note, 1958)

Bud Powell - pianoforte

Sam Jones - contrabbasso

Philly Joe Jones - batteria

The Scene Changes (The Amazing Bud Powell vol. 5)

(Blue Note, 1959)

Bud Powell - pianoforte

Paul Chambers - contrabbasso

Art Taylor - batteria 


\subsection{Presentazione della Scala}

Si riporta la scala Dorica Be-Bop di $C$ [11], nei moti ascendente e discendente, estesa lungo due ottave consecutive.

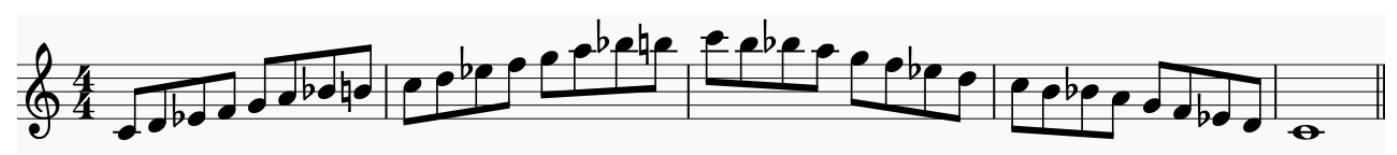

La scala appena presentata trova evidentemente applicazione in presenza dell'accordo $C-7$. [2]

L'aggiunta della settima maggiore (rispetto alla Dorica) è correlata all'ottenimento di un'interessante quadratura metrica. Eseguendo la scala a partire dalle note cordali (tonica, modale, dominante, sottotonica), queste risulteranno sempre posizionate in battere. Le tensioni residue (nona, undicesima, tredicesima) saranno ovviamente collocate in levare.

\subsection{Metabolizzare la Scala: Prime Frasi Elementari}

Uno dei primi esercizi in questa sede proposti, finalizzato ad una rapida metabolizzazione della scala, consiste nel suonare la Dorica Be-Bop in moto discendente, partendo dalle note cordali e raggiungendo, in ogni caso, la tonica (all'ottava inferiore rispetto a quella di partenza).

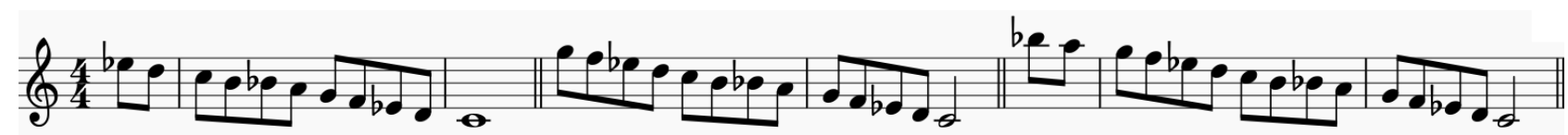

La frase con partenza dalla tonica è stata evidentemente omessa per brevità.

Per quanto affermato in precedenza, la quadratura metrica viene persa qualora la nota di partenza coincida con una delle tensioni. Il recupero metrico, tuttavia, è agevolmente ottenibile a mezzo di alcuni artifizi, assai diffusi nell'idioma Be-Bop. Il più banale consiste semplicemente nell'imporre un'anacrusi: in altri termini, la prima nota, che coincide con una delle tensioni residue, è suonata in levare. È inoltre possibile ribattere la tensione di partenza: coerentemente con la figurazione finora adoperata, pertanto, la frase inizierà, in tal caso, con due crome identiche consecutive. Alternativamente, è possibile raddoppiare la durata della tensione di partenza: sempre nel rispetto della figurazione adottata, la frase inizierà, in tal caso, con una semiminima. Inoltre, sono contemplabili soluzioni, talvolta decisamente più interessanti, basate su cromatismi ed approcci misti (alle note cordali pure).

Proponiamo di seguito tre semplicissime frasi con partenza dalla sopratonica (ovvero dalla nona).

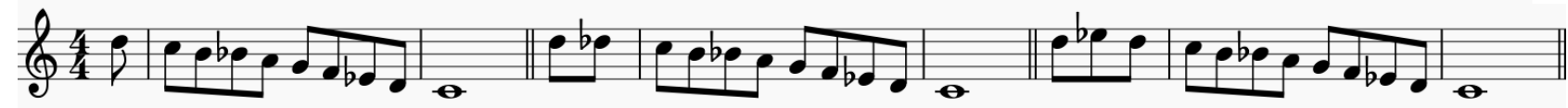


La prima è frase stata ottenuta imponendo un'anacrusi, la seconda costruendo (in anacrusi) un ponte cromatico verso la tonica, la terza imponendo (sempre in anacrusi, la cui durata è pari a $3 / 8$ al solo fine di collocare la tonica sul primo movimento dell'ultima battuta) un ponte cromatico verso la modale. A scopo squisitamente esercitativo, tutte le frasi proposte terminano sulla tonica (all'ottava inferiore rispetto a quella di partenza).

Proponiamo, adesso, tre semplicissime frasi con partenza dalla sottodominante (undicesima).

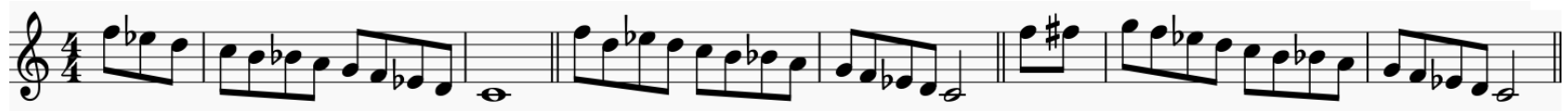

La prima frase è stata ottenuta imponendo un'anacrusi (la cui durata è pari a $3 / 8$ al solo fine di collocare la tonica sul primo movimento dell'ultima battuta), la seconda adoperando nulla più che un approccio misto alla modale (la sottodominante coincide con la nota d'approccio discendente alla modale), la terza costruendo (in anacrusi) un ponte cromatico verso la dominante. [3] Ancora una volta, le frasi terminano deliberatamente sulla tonica (all'ottava inferiore rispetto a quella di partenza).

Il secondo degli esempi sopra discussi ci offre l'opportunità di ricordare che, una volta definita la scala di riferimento, l'approccio alle note cordali pure (quelle costituenti la triade, essendo la settima soventemente qualificata come tensione fondamentale) è cromatico qualora ascendente, diatonico qualora discendente.

Proponiamo, infine, tre semplicissime frasi con partenza dalla sopradominante (sesta maggiore).

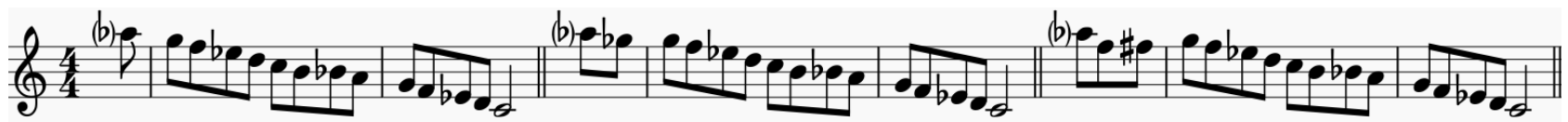

La prima frase è stata ottenuta imponendo un'anacrusi, la seconda adottando (in anacrusi) un approccio misto alla dominante, la terza adoperando (sempre in anacrusi, la cui durata è pari a $3 / 8$ al solo fine di collocare la tonica sul primo movimento dell'ultima battuta) un'enclosure [12] [13] [14] sulla dominante. Nuovamente, tutte le frasi sono intenzionalmente arrestate alla tonica. Il primo accidente in anacrusi, sebbene formalmente errato (nel rispetto della particolare scala considerata, l'approccio discendente alla dominante, diatonico per definizione, coincide con la sesta maggiore), viene spesso utilizzato al fine d'evitare la dissonanza tra settima minore (presente nel comping) e tredicesima. [3]

\subsection{Combinare Scale e Arpeggi}

Altro utilissimo esercizio consiste nell' esecuzione delle quadriadi terziane diatoniche deducibili dalla Scala Dorica. Nel caso particolare proposto in questa sede, considereremo accordi di settima arpeggiati [4], in moto ascendente, dedotti dall'armonizzazione [5] [6] [7] della Doria di $C$ ovvero, equivalentemente, della Ionica di $B^{b}$.

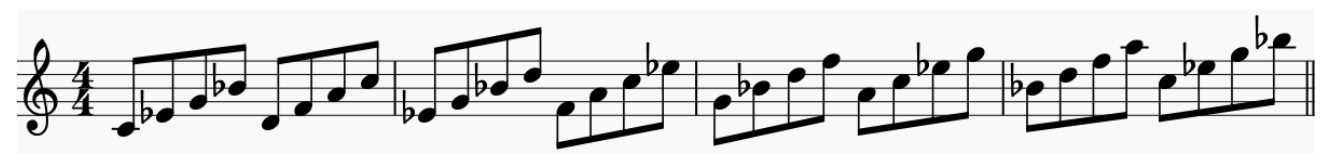

Le quadriadi terziane diatoniche possono essere agevolmente combinate con le frasi costruite sulla Dorica Be-Bop: molto semplicemente, l'ultima nota dell'arpeggio rappresenterà l'elemento di 
congiunzione tra il suddetto e la scala. A titolo d'esempio, la quarta nota dell'arpeggio ascendente di $E^{b}$ maj7 è $D$ (simultaneamente sensibile rispetto a $E^{b}$ e sopratonica rispetto a $C$ ): pertanto, il suddetto arpeggio potrà agganciarsi a qualsivoglia frase, costruita sulla Dorica Be-Bop, che preveda partenza dalla sopratonica (nona). Assai spesso, inoltre, si preferisce inserire un approccio ascendente (e quindi, per definizione, cromatico) alla prima nota dell'arpeggio. Proponiamo, di seguito, alcuni esempi (uno per ognuna delle note cordali) al fine d'illustrare qualitativamente il modo in cui le quadriadi terziane diatoniche possano combinarsi con la scala Dorica Be-Bop.
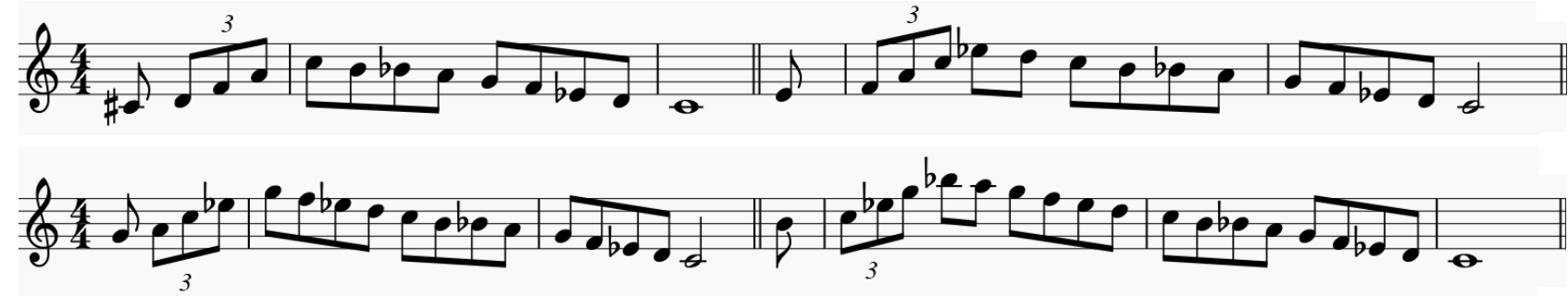

La prima nota dell'arpeggio è sempre approcciata. Le prime tre note costituiscono una terzina: l'adozione di tale gruppo irregolare, sebbene tutto fuorché strettamente necessaria, costituisce elemento distintivo dell'idioma Be-Bop. [3] Come sempre in questa sede, le frasi proposte terminano sulla tonica (all'ottava inferiore rispetto a quella di partenza). E d'uopo evidenziare come, al netto di quello costruito sulla sottotonica (settima minore), gli esempi appena riportati possiedano valore prevalentemente esercitativo.

Proponiamo alcuni esempi più articolati costruiti sulle tensioni (due per ognuna delle tensioni).
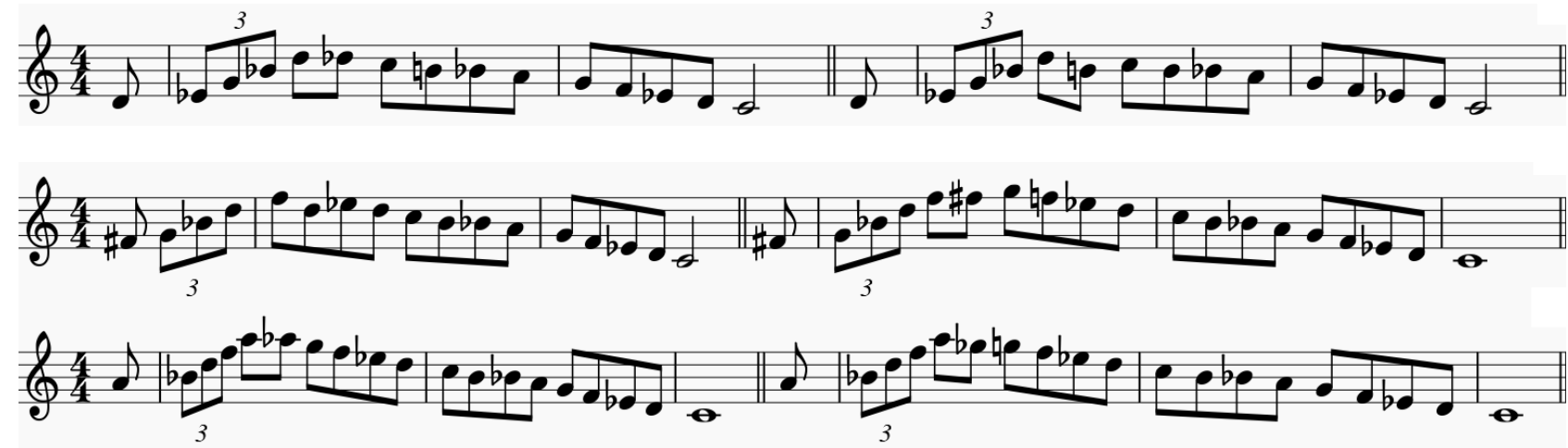

\subsection{Osservazioni Finali}

A rigore, la scala Dorica Be-Bop propriamente intesa, sebbene di scarso utilizzo, prevede l'aggiunta della terza maggiore (rispetto alla Dorica) e non della sensibile (settima maggiore). Si riporta, di seguito, la scala Dorica Be-Bop propriamente intesa di $C$, nei moti ascendente e discendente, estesa lungo due ottave consecutive.

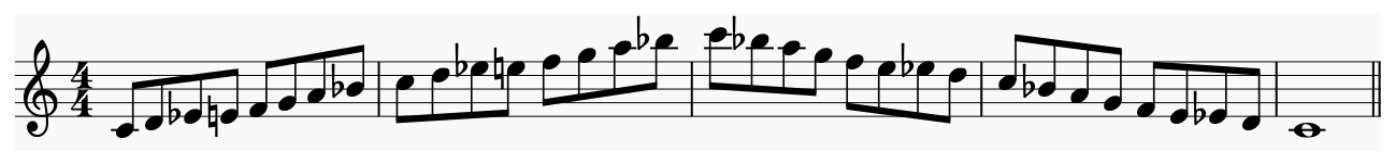

È agevole verificare come le note costituenti la scala appena presentata coincidano, nel caso specifico, con quelle della Dominante Be-Bop di $F$ : non a caso, in presenza di progressioni armoniche assai 
dense (quattro accordi per misura sulle ballad ovvero due sugli up-tempo), l'accordo $C-7$, seguito da F7, si contrae in quest'ultimo (dando luogo al fenomeno opposto a quello dell'espansione). [8] [9] Generalizzando, si può affermare come la Dorica Be-Bop propriamente intesa scaturisca dal quinto grado della Dominante Be-Bop (in termini forzatamente modali, il Dorico Be-Bop propriamente inteso può essere considerato come il quinto dei modi derivanti dalla Dominante Be-Bop). 


\section{La Scala Minore Naturale Be-Bop}

\subsection{Presentazione della Scala}

Si riporta la scala Minore Naturale Be-Bop di $C$ [15], nei moti ascendente e discendente, estesa lungo due ottave consecutive.

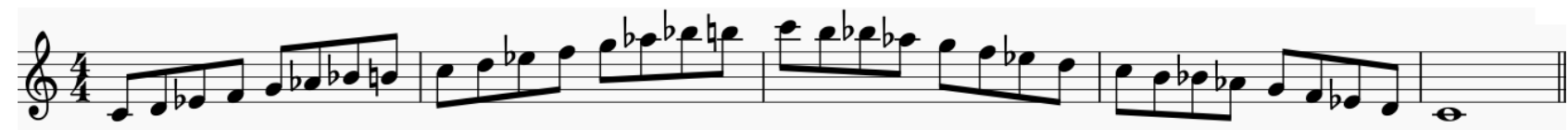

La scala appena presentata trova evidentemente applicazione in presenza dell'accordo $C-7$. [2]

L'aggiunta della settima maggiore (rispetto alla Minore Naturale) è correlata all'ottenimento di un'interessante quadratura metrica. Eseguendo la scala a partire dalle note cordali (tonica, modale, dominante, sottotonica), queste risulteranno sempre posizionate in battere. Le tensioni residue (nona, undicesima, tredicesima) saranno ovviamente collocate in levare.

\subsection{Metabolizzare la Scala: Prime Frasi Elementari}

Uno dei primi esercizi in questa sede proposti, finalizzato ad una rapida metabolizzazione della scala, consiste nel suonare la Minore Naturale Be-Bop in moto discendente, partendo dalle note cordali e raggiungendo, in ogni caso, la tonica (all'ottava inferiore rispetto a quella di partenza).

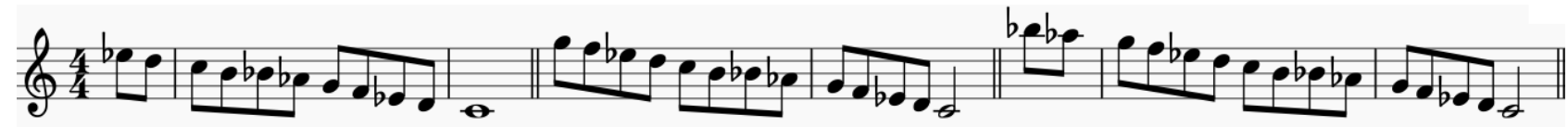

La frase con partenza dalla tonica è stata evidentemente omessa per brevità.

Per quanto affermato in precedenza, la quadratura metrica viene persa qualora la nota di partenza coincida con una delle tensioni. Il recupero metrico, tuttavia, è agevolmente ottenibile a mezzo di alcuni artifizi, assai diffusi nell'idioma Be-Bop. Il più banale consiste semplicemente nell'imporre un'anacrusi: in altri termini, la prima nota, che coincide con una delle tensioni residue, è suonata in levare. È inoltre possibile ribattere la tensione di partenza: coerentemente con la figurazione finora adoperata, pertanto, la frase inizierà, in tal caso, con due crome identiche consecutive. Alternativamente, è possibile raddoppiare la durata della tensione di partenza: sempre nel rispetto della figurazione adottata, la frase inizierà, in tal caso, con una semiminima. Inoltre, sono contemplabili soluzioni, talvolta decisamente più interessanti, basate su cromatismi ed approcci misti (alle note cordali pure).

Proponiamo di seguito tre semplicissime frasi con partenza dalla sopratonica (ovvero dalla nona).

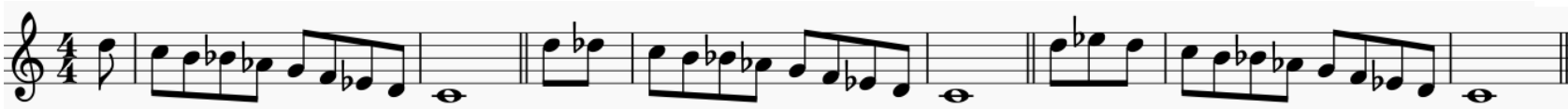


La prima è frase stata ottenuta imponendo un'anacrusi, la seconda costruendo (in anacrusi) un ponte cromatico verso la tonica, la terza imponendo (sempre in anacrusi, la cui durata è pari a 3/8 al solo fine di collocare la tonica sul primo movimento dell'ultima battuta) un ponte cromatico corto verso la modale (un approccio cromatico ascendente alla modale). A scopo squisitamente esercitativo, tutte le frasi proposte terminano sulla tonica (all'ottava inferiore rispetto a quella di partenza).

Proponiamo, adesso, tre semplici frasi con partenza dalla sottodominante (ovvero dall'undicesima).

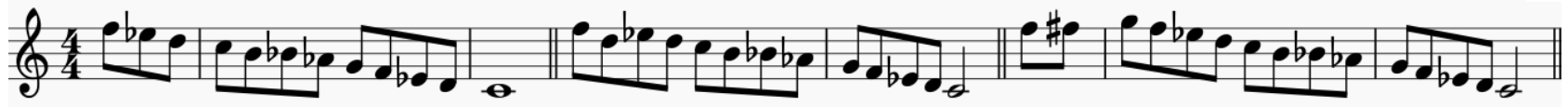

La prima frase è stata ottenuta imponendo un'anacrusi (la cui durata è pari a $3 / 8$ al solo fine di collocare la tonica sul primo movimento dell'ultima battuta), la seconda adoperando nulla più che un approccio misto alla modale (la sottodominante coincide con la nota d'approccio discendente alla modale), la terza costruendo (in anacrusi) un ponte cromatico verso la dominante. [3] Ancora una volta, le frasi terminano deliberatamente sulla tonica (all'ottava inferiore rispetto a quella di partenza).

Il secondo degli esempi sopra discussi ci offre l'opportunità di ricordare che, una volta definita la scala di riferimento, l'approccio alle note cordali pure (quelle costituenti la triade, essendo la settima soventemente qualificata come tensione fondamentale) è cromatico qualora ascendente, diatonico qualora discendente.

Proponiamo, infine, tre semplicissime frasi con partenza dalla sopradominante (sesta minore).

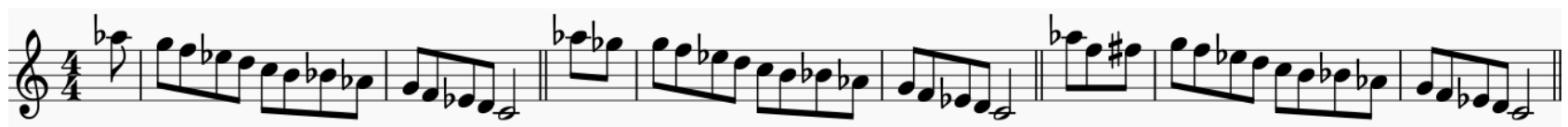

La prima frase è stata ottenuta imponendo un'anacrusi, la seconda adottando (in anacrusi) un approccio misto alla dominante, la terza adoperando (sempre in anacrusi, la cui durata è pari a $3 / 8$ al solo fine di collocare la tonica sul primo movimento dell'ultima battuta) un'enclosure [12] [13] [14] sulla dominante. Nuovamente, tutte le frasi sono intenzionalmente arrestate alla tonica.

\subsection{Combinare Scale e Arpeggi}

Altro utilissimo esercizio consiste nell' esecuzione delle quadriadi terziane diatoniche deducibili dalla Scala Minore Naturale. Nel caso particolare proposto in questa sede, considereremo accordi di settima arpeggiati [4], in moto ascendente, dedotti dall'armonizzazione [5] [6] [7] della Minore Naturale (Eolia) di $C$ ovvero, equivalentemente, della Ionica di $E^{b}$.

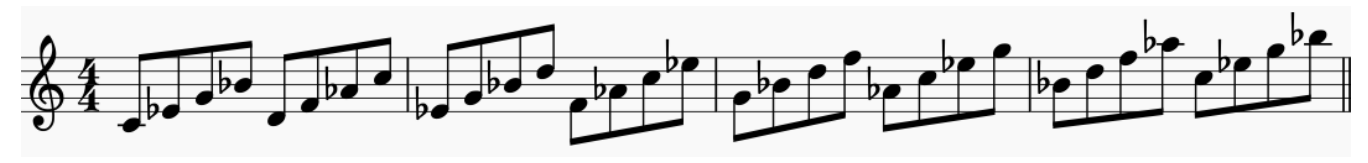

Le quadriadi terziane diatoniche possono essere agevolmente combinate con le frasi costruite sulla Minore Naturale Be-Bop: molto semplicemente, l'ultima nota dell'arpeggio rappresenterà l'elemento di congiunzione tra il suddetto e la scala. A titolo d'esempio, la quarta nota dell'arpeggio ascendente di $G-7$ è $F$ (simultaneamente sottotonica rispetto a $G$ e sottodominante rispetto a $C$ ): pertanto, il suddetto arpeggio potrà agganciarsi a qualsivoglia frase, costruita sulla Minore Naturale Be-Bop, che preveda partenza dalla sottodominante (undicesima). 
Assai spesso, inoltre, si preferisce inserire un approccio ascendente (e quindi, per definizione, cromatico) alla prima nota dell'arpeggio. Proponiamo ora alcuni esempi (uno per ognuna delle note cordali), al fine d'illustrare qualitativamente il modo in cui le quadriadi terziane diatoniche possano combinarsi con la scala Minore Naturale Be-Bop.

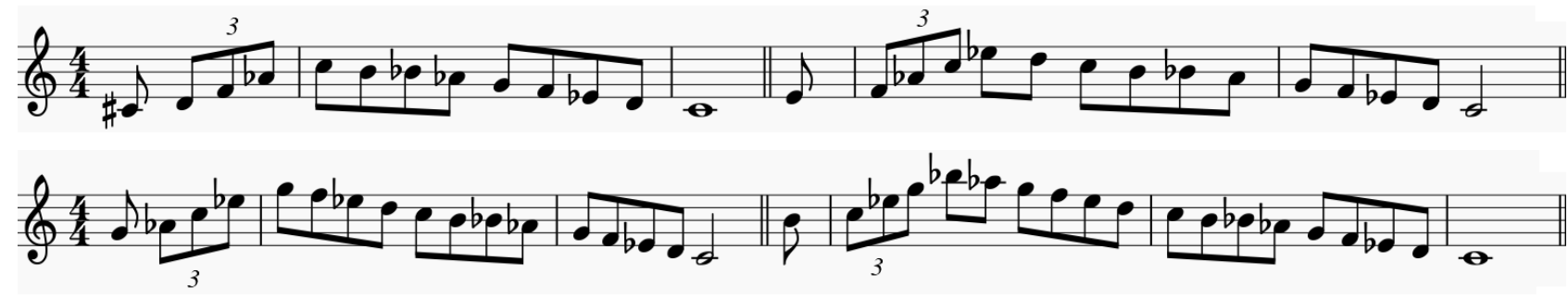

La prima nota dell'arpeggio è sempre approcciata. Le prime tre note costituiscono una terzina: l'adozione di tale gruppo irregolare, sebbene tutto fuorché strettamente necessaria, costituisce elemento distintivo dell'idioma Be-Bop. [3] Come sempre in questa sede, le frasi proposte terminano sulla tonica (all'ottava inferiore rispetto a quella di partenza). E d'uopo evidenziare come, al netto di quello costruito sulla sottotonica (settima minore), gli esempi appena riportati possiedano valore prevalentemente esercitativo.

Proponiamo alcuni esempi più articolati costruiti sulle tensioni (due per ognuna delle tensioni).
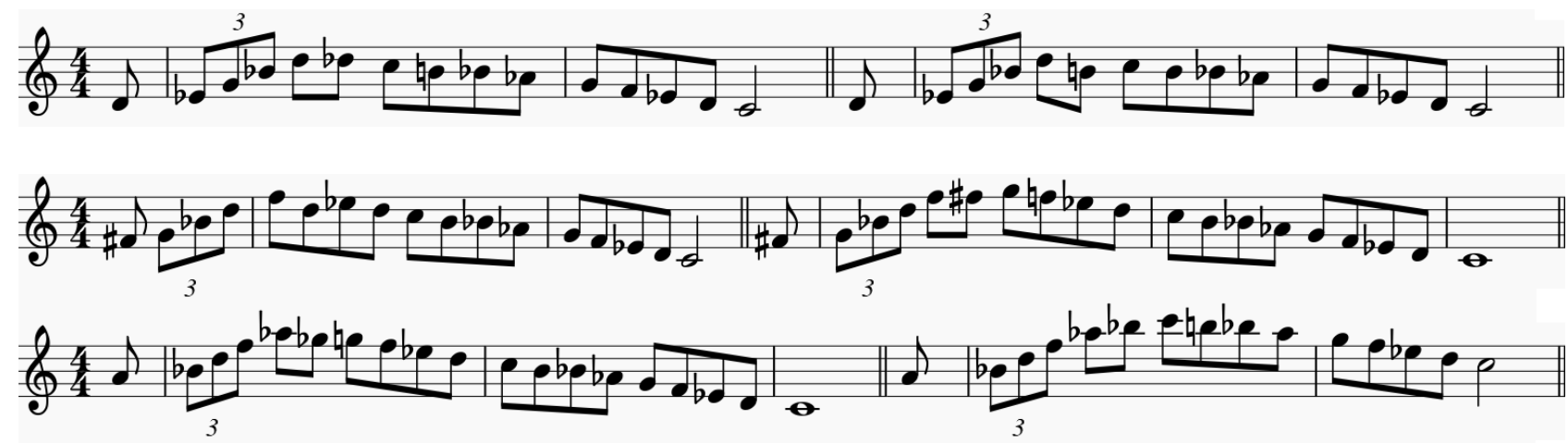

\subsection{Osservazioni Finali}

È agevole verificare come le note costituenti la scala Minore Naturale Be-Bop di $C$ coincidano con quelle della Maggiore Be-Bop di $E^{b}$ (si veda il capitolo successivo). Generalizzando, si potrebbe affermare come la scala Maggiore Be-Bop scaturisca dal terzo grado della Minore Naturale Be-Bop: alternativamente, in termini forzatamente modali, lo Ionico Be-Bop potrebbe essere considerato come il terzo dei modi derivanti dalla Minore Naturale (ovvero Eolia) Be-Bop.

È interessante sottolineare come la scala Minore Naturale Be-Bop, adoperata tal quale, possa essere serenamente impiegata (a patto di mantenere in battere le note cordali) su cadenze autentiche (armonicamente perfette), pure ovvero composte, nonché sul classico turnaround minore (cadenze e turnaround sono da considerarsi di derivazione Eolia).

Tale caratteristica può essere sfruttata a prescindere dalla densità armonica della particolare progressione, rivelandosi utilissima qualora applicata alle sequenze strette (due accordi per misura) ovvero addirittura strettissime (quattro accordi per misura, come spesso accade nelle ballad). 


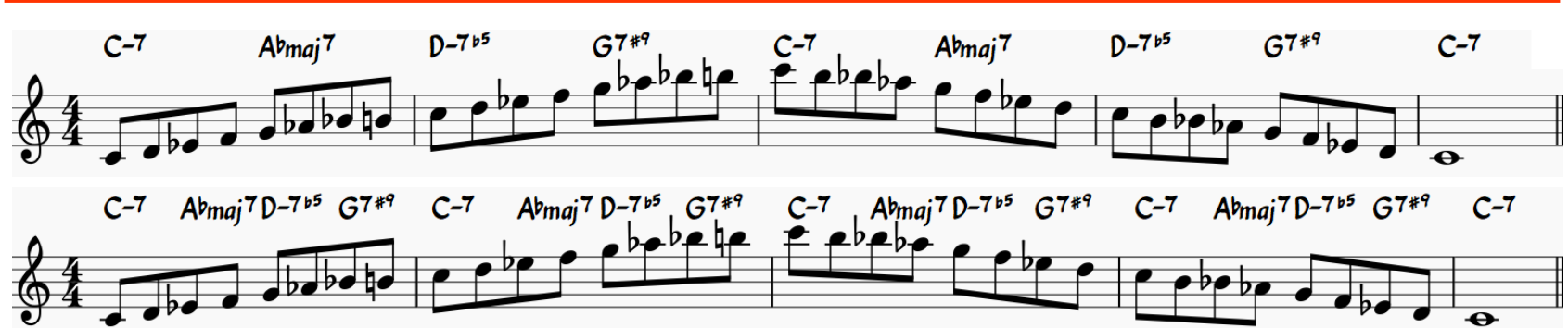

Assai evidentemente, l'accordo $G-7$ è stato immediatamente assoggettato a sostituzione per dominante secondaria (funzionale) [7] [8] [9]. Il motivo è molto semplice: l'adozione dell'accordo di seconda specie è fenomeno raro nonché di scarsissimo valore jazzistico. Nel rispetto dell'armonizzazione (della scala Eolia), l'accordo di prima specie, scaturito dalla sostituzione di cui sopra, è stato formalmente provvisto della nona diesis (enarmonicamente coincidente con la terza minore). A rigore, al fine di garantire una perfetta aderenza tra il turnaround minore modificato e la scala Minore Naturale Be-Bop, l'accordo di prima specie dovrebbe essere altresì provvisto della tredicesima bemolle. Nondimeno, la scala è di prassi adoperabile, nella sua interezza, anche qualora l'accordo di prima specie sia provvisto della sola nona bemolle. Seguono tre semplicissime applicazioni (scala Minore Naturale Be-Bop, nei moti ascendente e discendente, con estensione alla singola ottava, riprodotta con partenza da modale, dominante e sottotonica).

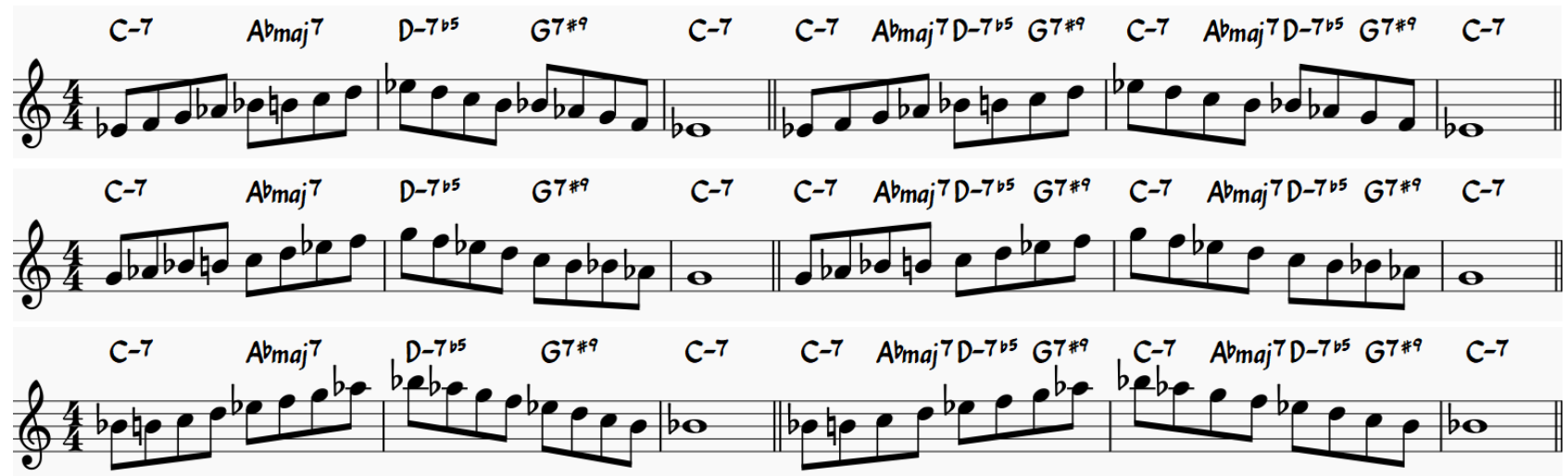

Per quanto banali, gli esempi appena proposti dovrebbero agevolare la comprensione d'un concetto tanto elementare quanto di fondamentale importanza: qualora in presenza delle progressioni armoniche richiamate all'inizio del paragrafo, l'improvvisatore è autorizzato ad adoperare la scala Minore Naturale Be-Bop senza curarsi del particolare accordo. L'unica premura, infatti, consisterà nel mantenere in battere le note cordali (tonica, modale, dominante, sottotonica), qualificabili come punti di controllo in corrispondenza dei quali poter liberamente ascendere ovvero discendere.

Proponiamo di seguito un esempio chiarificatore, applicato ad armonia stretta e strettissima.

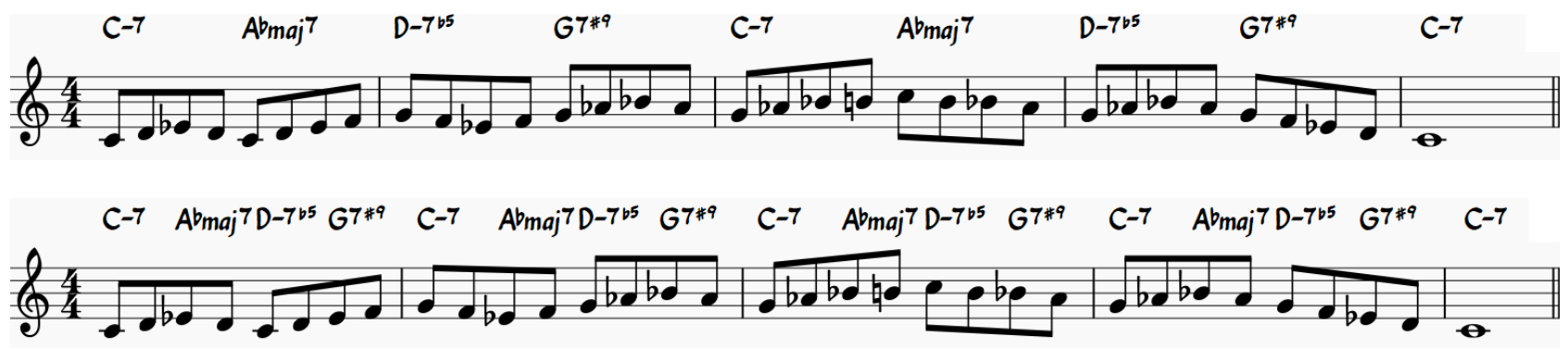




\section{La Scala Maggiore Be-Bop}

\subsection{Presentazione della Scala}

Si riporta di seguito la scala Maggiore Be-Bop di $C$ [16], nei moti ascendente e discendente, estesa lungo due ottave consecutive.

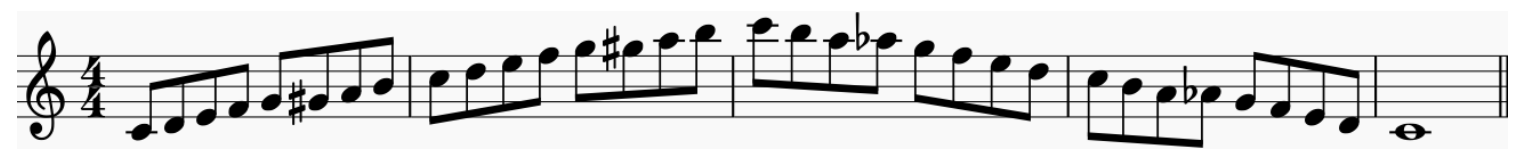

Tale scala (il cui utilizzo, a rigore, risulterebbe formalmente vincolato all'accordo $C 6$ ) è diffusamente adoperata in presenza dell'accordo Cmaj7. [2]

L'aggiunta della sesta minore è fondamentalmente correlata all'ottenimento di un'interessante quadratura metrica. Eseguendo la scala a partire dalle note cordali pure (tonica, modale, dominante) e dalla sesta maggiore, queste risulteranno sempre posizionate in battere. Le tensioni residue (settima, nona, undicesima,) saranno collocate in levare.

\subsection{Metabolizzare la Scala: Prime Frasi Elementari}

Uno dei primi esercizi in questa sede proposti, finalizzato ad una rapida metabolizzazione della scala, consiste nel suonare la Maggiore Be-Bop in moto discendente, partendo dalle note cordali pure e dalla sesta, raggiungendo, in ogni caso, la tonica (all'ottava inferiore rispetto a quella di partenza).

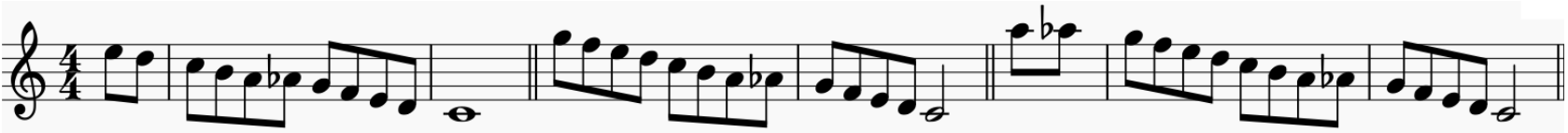

La frase con partenza dalla tonica è stata evidentemente omessa per brevità.

Per quanto affermato in precedenza, la quadratura metrica viene persa qualora la nota di partenza coincida con una delle tensioni residue (la settima, considerata in questa sede quale tensione fondamentale, la nona l'undicesima). Il recupero metrico, tuttavia, è agevolmente ottenibile a mezzo di alcuni artifizi, assai diffusi nell'idioma Be-Bop. Il più banale consiste semplicemente nell'imporre un'anacrusi: in altri termini, la prima nota, che coincide con una delle tensioni residue, è suonata in levare. È inoltre possibile ribattere la tensione di partenza: coerentemente con la figurazione finora adoperata, pertanto, la frase inizierà, in tal caso, con due crome identiche consecutive. Alternativamente, è possibile raddoppiare la durata della tensione di partenza: sempre nel rispetto della figurazione adottata, la frase inizierà, in tal caso, con una semiminima. Inoltre, sono contemplabili soluzioni, talvolta decisamente più interessanti, basate su cromatismi ed approcci misti (alle note cordali pure).

Proponiamo di seguito tre semplicissime frasi con partenza dalla sopratonica (ovvero dalla nona). 


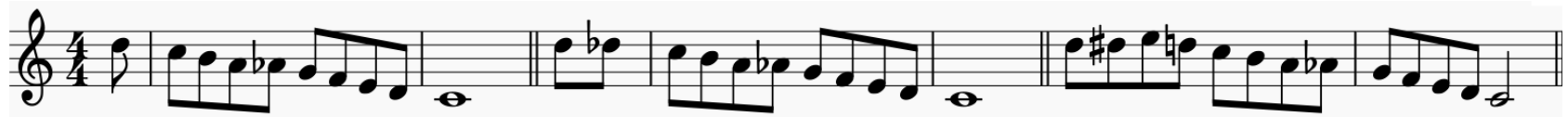

La prima è frase stata ottenuta imponendo un'anacrusi, la seconda costruendo (in anacrusi) un ponte cromatico verso la tonica, la terza adoperando un ponte cromatico verso la modale. [3] A scopo squisitamente esercitativo, tutte le frasi proposte terminano sulla tonica (all'ottava inferiore rispetto a quella di partenza).

Proponiamo tre semplicissime frasi con partenza dalla sottodominante (ovvero dall'undicesima).

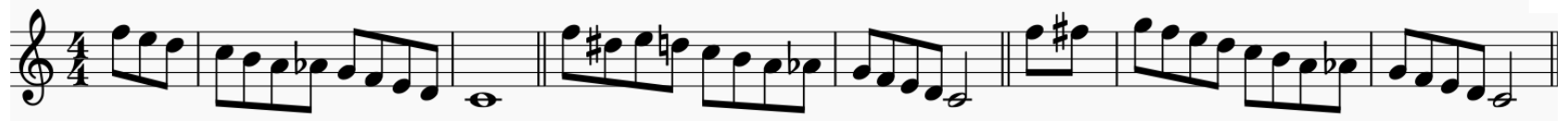

La prima frase è stata ottenuta imponendo un'anacrusi (la cui durata è pari a $3 / 8$ al solo fine di collocare la tonica sul primo movimento dell'ultima battuta), la seconda adoperando nulla più che un approccio misto alla modale (la sottodominante coincide con la nota d'approccio discendente alla modale), la terza costruendo (in anacrusi) un ponte cromatico verso la dominante. [3] Ancora una volta, le frasi terminano deliberatamente sulla tonica (all'ottava inferiore rispetto a quella di partenza).

Il secondo degli esempi sopra discussi ci offre l'opportunità di ricordare che, una volta definita la scala di riferimento, l'approccio alle note cordali pure (quelle costituenti la triade, essendo la settima soventemente ritenuta, come già sottolineato in precedenza, tensione fondamentale) è cromatico qualora ascendente, diatonico qualora discendente.

Proponiamo, infine, tre semplicissime frasi con partenza dalla sensibile (settima maggiore).

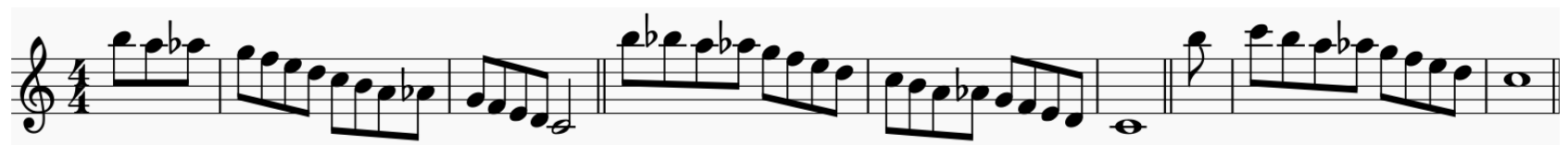

La prima frase è stata ottenuta imponendo un'anacrusi (la cui durata è pari a $3 / 8$ al solo fine di collocare la tonica sul terzo movimento dell'ultima battuta), la seconda costruendo un ponte cromatico verso la sopradominante (e quindi, considerata la struttura della scala, verso la dominante), la terza effettuando (in anacrusi) un approccio cromatico ascendente alla tonica (all'ottava superiore). [3] Nuovamente, tutte le frasi sono intenzionalmente arrestate alla tonica.

Per completezza, proponiamo tre utilissime frasi di pubblico dominio [12] [13] [14] [17] costruite sulla scala Maggiore Be-Bop.

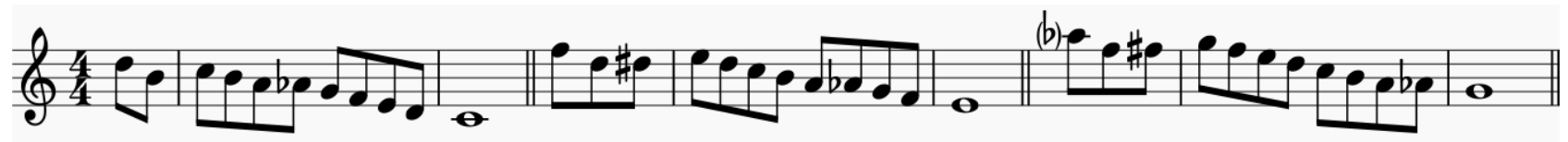

La prima è ottenuta adottando (in anacrusi) un approccio misto alla tonica, la seconda imponendo (in anacrusi) un'enclosure [12] [13] [14] sulla modale, la terza imponendo (in anacrusi) un'enclosure sulla dominante. Nell'ultimo caso, l'accidente iniziale è da ritenersi opzionale siccome l'approccio discendente alla modale, diatonico per definizione, è rappresentato dalla sesta maggiore, nel caso in cui si consideri, quale scala di riferimento, la Ionica, ovvero dalla sesta minore, nel caso in cui ci si riferisca alla scala Maggiore Be-Bop. A guisa di promemoria per l'esecutore, le frasi appena proposte terminano proprio sulle note cui l'approccio misto e le anacrusi vengono inizialmente applicate. 


\subsection{Combinare Scale e Arpeggi}

Altro utilissimo esercizio consiste nell'esecuzione delle quadriadi terziane diatoniche relative alla tonalità di pertinenza. Nel caso in questa sede proposto (stiamo ipotizzando d'improvvisare su Cmaj7), considereremo accordi di settima arpeggiati [4], in moto ascendente, dedotti dall'armonizzazione [5] [6] [7] della Ionica di $C$.

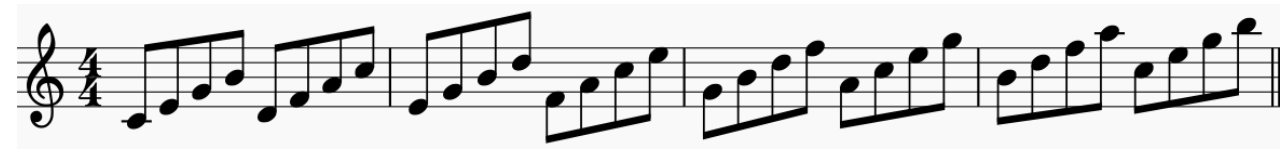

Le quadriadi terziane diatoniche possono essere agevolmente combinate con le frasi costruite sulla Maggiore Be-Bop: molto semplicemente, l'ultima nota dell'arpeggio rappresenterà l'elemento di congiunzione tra il suddetto e la scala. A titolo d'esempio, la quarta nota dell'arpeggio ascendente di $A \min 7$ è $G$ (simultaneamente sottotonica rispetto ad $A$ e dominante rispetto a $C$ ): pertanto, il suddetto arpeggio potrà agganciarsi ad una qualsivoglia frase, costruita sulla Maggiore Be-Bop, che preveda partenza dalla quinta. Assai spesso, inoltre, si preferisce approcciare ascendentemente (e quindi, per definizione, cromaticamente) la prima nota dell'arpeggio. Proponiamo, di seguito, alcuni esempi (uno per ognuna delle note cordali pure, costituenti la triade maggiore di $C$, ed uno per la sopradominante) al fine d'illustrare qualitativamente il modo in cui le quadriadi terziane diatoniche possano combinarsi con la scala Maggiore Be-Bop.
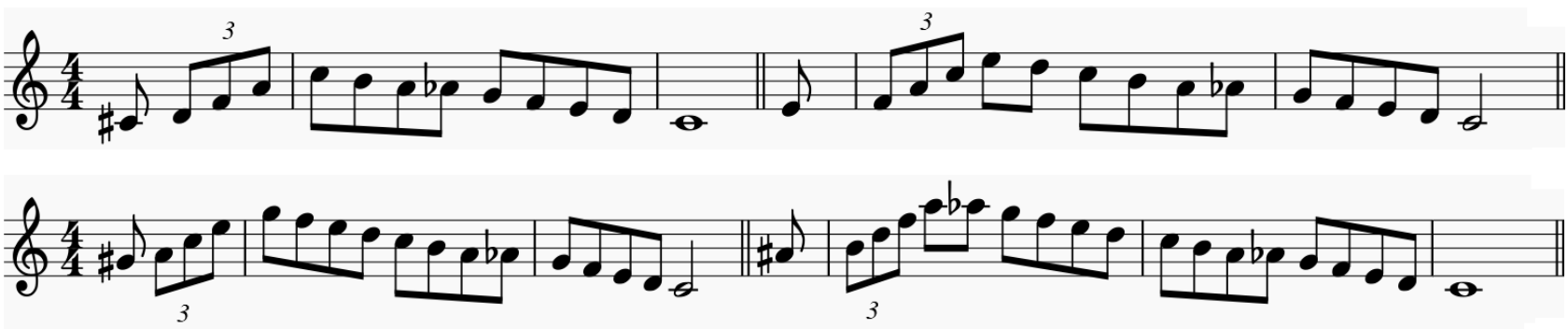

La prima nota dell'arpeggio è sempre approcciata. Le prime tre note costituiscono una terzina: l'adozione di tale gruppo irregolare, sebbene tutto fuorché strettamente necessaria, costituisce elemento distintivo dell'idioma Be-Bop. [3] Come quasi sempre in questa sede, le frasi proposte terminano sulla tonica (all'ottava inferiore rispetto a quella di partenza).

Proponiamo una serie di esempi più articolati costruiti sulle tensioni residue (due frasi per ognuno dei gradi tensivi).
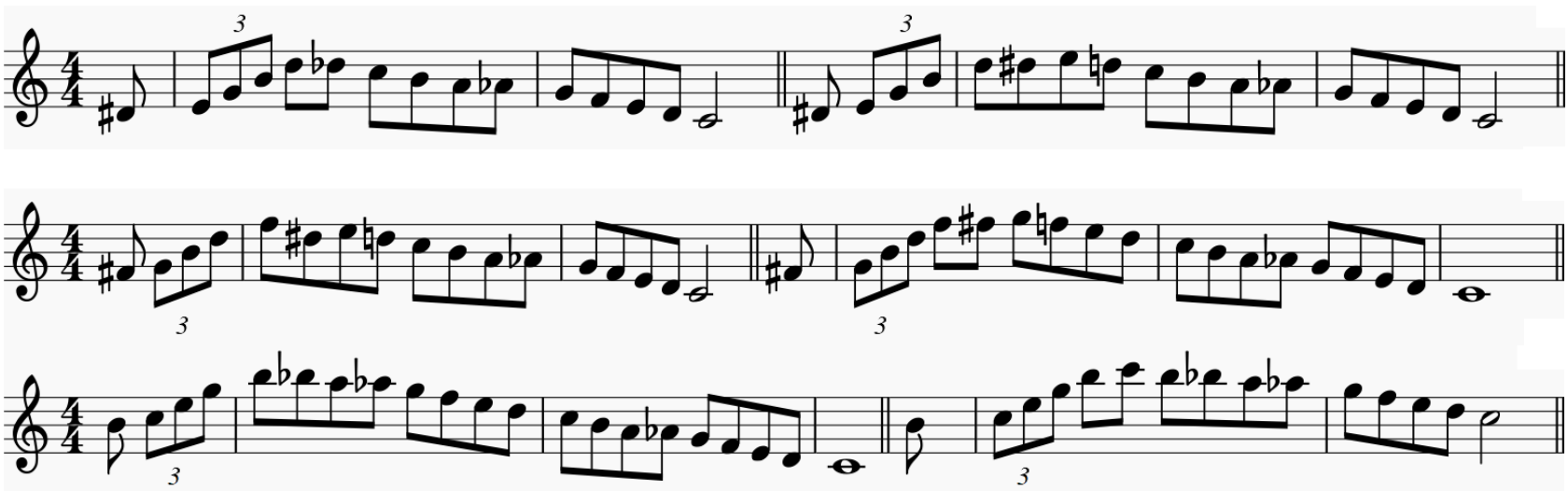


\subsection{Osservazioni Finali}

È interessante sottolineare come la scala Maggiore Be-Bop, adoperata tal quale, possa essere impiegata (a patto di mantenere in battere tonica, modale, dominante e sopradominante) su cadenze autentiche pure (V7 Imaj7) e composte (II-7 V7 | Imaj7), nonché sui classici turnaround maggiori.

Tale caratteristica può essere sfruttata a prescindere dalla densità armonica della particolare progressione, rivelandosi utilissima qualora applicata alle sequenze strette (due accordi per misura) ovvero addirittura strettissime (quattro accordi per misura, come spesso accade nelle ballad).

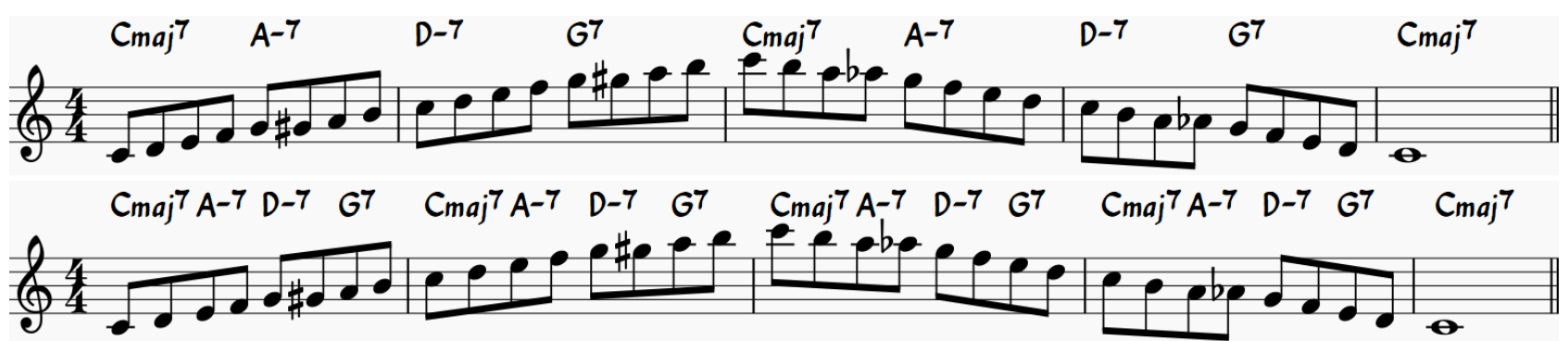

Seguono tre semplicissime applicazioni (scala Maggiore Be-Bop, nei moti ascendente e discendente, con estensione alla singola ottava, riprodotta con partenza da modale, dominante e sopradominante).

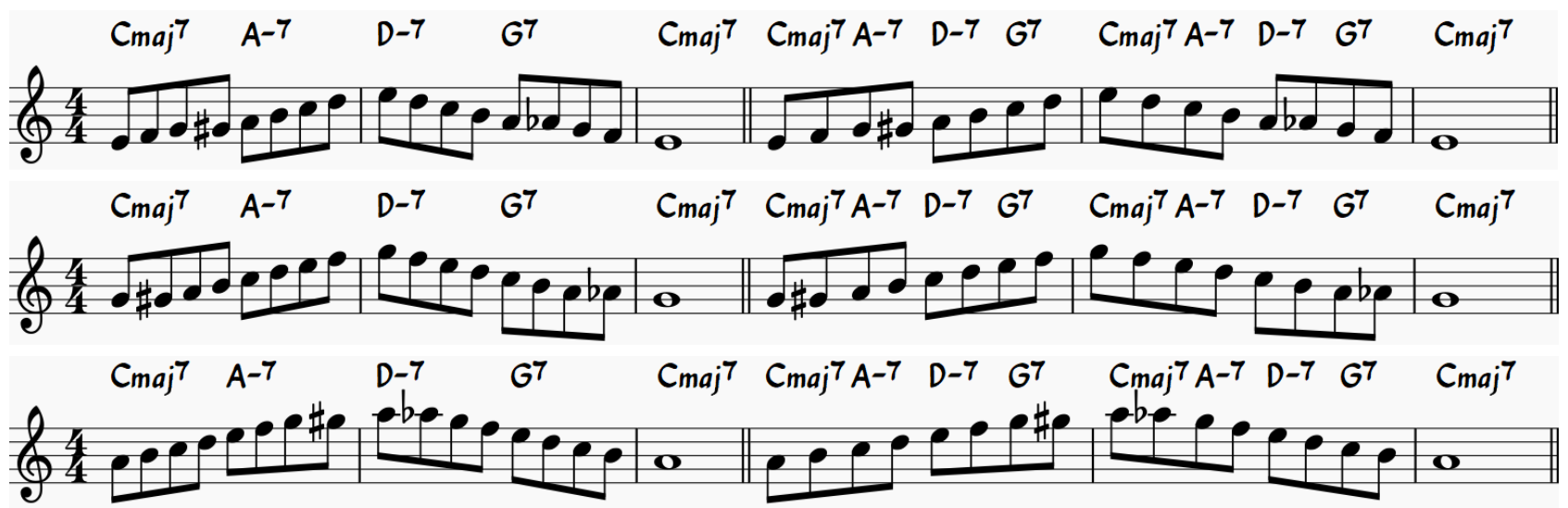

Gli esempi appena proposti suggeriscono come, qualora in presenza delle progressioni armoniche richiamate all'inizio del paragrafo, l'improvvisatore sia legittimato ad adoperare la scala Maggiore Be-Bop senza curarsi del particolare accordo. L'unica premura, infatti, consisterà nel mantenere in battere le note caratteristiche della scala (tonica, modale, dominante, sopradominante), qualificabili come punti di controllo in corrispondenza dei quali poter liberamente ascendere ovvero discendere.

Proponiamo di seguito un esempio chiarificatore, applicato ad armonia stretta e strettissima.

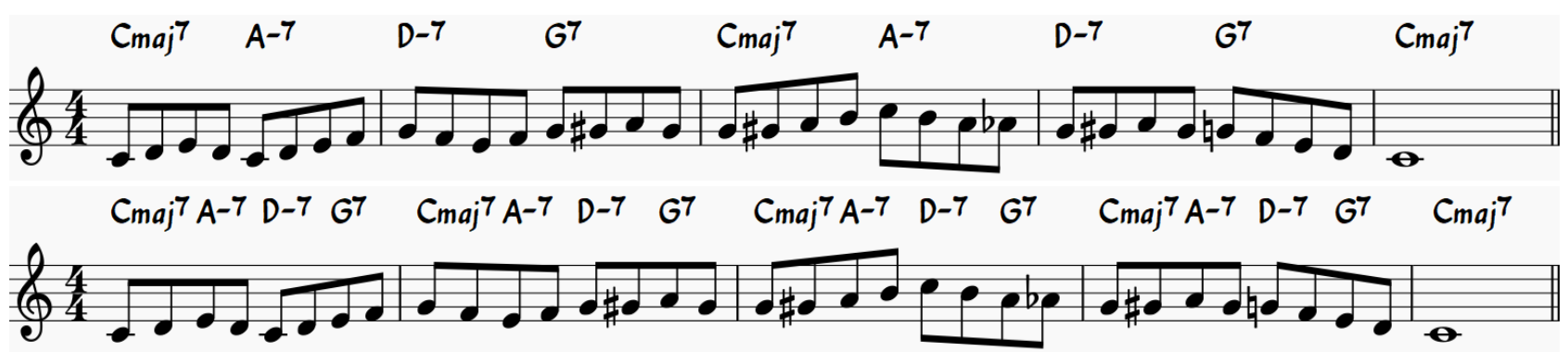


Per quanto scontato, sottolineiamo come l'accordo $A-7$ possa essere assoggettato a sostituzione per dominante secondaria (funzionale) [7] [8] [9], senza svilire in alcuna misura il principio poc'anzi esposto. In altri termini, $A-7$ può essere serenamente rimpiazzato da $A 7$ (eventualmente provvisto della nona diesis e/o della tredicesima bemolle). Com'è agevole verificare, nonostante la sostituzione di cui sopra implichi una fluttuazione locale del centro tonale, l'improvvisatore potrà continuare ad avvalersi della scala Maggiore Be-Bop preoccupandosi semplicemente, ancora una volta, di mantenere in battere tonica, modale, dominante e sopradominante. Per completezza, specifichiamo come la modalità improvvisativa appena descritta (si vedano anche le osservazioni finali al capitolo precedente) venga comunemente definita orizzontale: di contro, l'improvvisazione verticale richiede di porre l'attenzione su ognuno degli accordi della progressione, prescindendo, almeno in certa misura, dai legami armonici. 

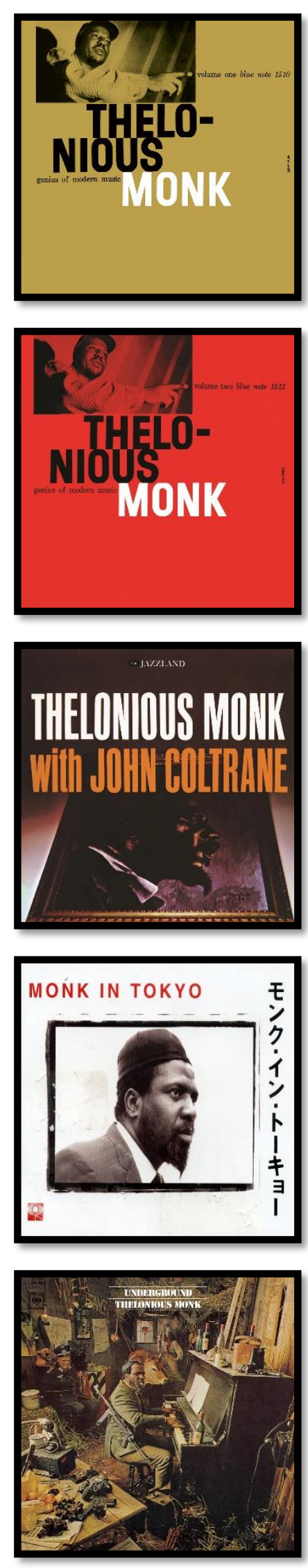

\section{Genius of Modern Music: Volume 1}

(Blue Note, 1951 - 1989)

Idrees Sulieman / George Taitt - tromba

Danny Quebec West / Sahib Shihab - sassofono contralto

Billy Smith - sassofono tenore

Thelonious Monk - pianoforte

Gene Ramey / Bob Paige - contrabbasso

Art Blakey - batteria

\section{Genius of Modern Music: Volume 2}

(Blue Note, 1952 - 1989)

Kenny Dorham - tromba

Lou Donaldson / Sahib Shihab - sassofono contralto

Lucky Thompson - sassofono tenore

Milt Jackson - vibrafono

Thelonious Monk - pianoforte

Al McKibbon / Nelson Boyd - contrabbasso

Art Blakey / Max Roach - batteria

\section{Thelonious Monk with John Coltrane}

(JazzLand, 1961)

Ray Copeland - tromba

Gigi Gryce - sassofono contralto

John Coltrane / Coleman Hawkins - sassofono tenore

Thelonious Monk - pianoforte

Wilbur Ware - contrabbasso

Art Blakey / Shadow Wilson - batteria

\section{Monk in Tokio}

(Columbia, 1963)

Charlie Rouse - sax

Thelonious Monk - piano

Butch Warren - contrabbasso

Frankie Dunlop - batteria

\section{Underground}

(Columbia, 1968)

Charlie Rouse - sax

Thelonious Monk - piano

Larry Gales - contrabbasso

Ben Riley - batteria

Jon Hendricks - voce 


\section{Il Linguaggio Articolato: Costruire sulle Triadi}




\section{Introduzione alla Seconda Sezione}

I capitoli di questa sezione (tre in tutto) sono espressamente dedicati all'esposizione di un'interessante metodologia improvvisativa, formalizzata dall'autore nel corso degli ultimi due anni, il cui fine principale risiede nel consentire il rapido sviluppo d'un fraseggio sufficientemente articolato, anche nel caso in cui l'aspirante improvvisatore risulti sprovvisto d'un ragionevole background jazzistico.

Si parte dalla metabolizzazione di una "frase fondamentale", che varia in funzione della tipologia di accordo, confezionata in modo tale da risultare, già di per sé, pienamente rappresentativa d'una fetta assai generosa dei costrutti boppistici di pubblico dominio. La "frase fondamentale" è costruita intorno alle note cordali pure, rivelandosi agevolmente riproducibile, per "traslazione", a partire da ognuna di esse. La "frase fondamentale" può essere adoperata in forma frammentata, imponendo banali tagli, altresì prestandosi a numerose modificazioni per aggiunta di "parole elementari" (generalmente, nulla più che banali ponti cromatici tra terze diatoniche).

Al fine d'agevolare la combinazione di frasi e parole, tutti i pentagrammi della sezione sono numerati progressivamente. La numerazione è azzerata all'inizio d'ogni capitolo.

I capitoli possiedono struttura simile, ma non identica.

Il Primo Paragrafo è dedicato alla presentazione della "frase fondamentale", trascritta in modo tale da evidenziare le note cordali pure: tale caratteristica è adoperata nell'intera sezione.

Il Secondo Paragrafo è rivolto alla focalizzazione dell'attenzione sulle note della triade, riferimenti imprescindibili cui l'improvvisatore sarà tenuto ad ancorarsi.

Il contenuto dei paragrafi successivi è variabile. Nei Capitoli 6 e 8, Terzo e Quarto Paragrafo sono dedicati all'introduzione, rispettivamente, di settima e nona; nel Capitolo 7, di contro, si è preferito consacrare il Terzo Paragrafo all'immediata trattazione delle tensioni ammissibili nella loro totalità, descrivendo i ponti cromatici realizzabili tra terze diatoniche, e il Quarto all'applicazione del metodo in caso di tonicizzazione. Nel Capitolo 6, il Quinto Paragrafo tratta assai brevemente l'arricchimento delle frasi ottenute; nei Capitoli 7 e 8 , il medesimo paragrafo è rivolto, rispettivamente, allo studio dei cosiddetti "accordi parenti" e all'applicazione del metodo in caso di tonicizzazione ovvero in presenza di interi "turnaround".

L'ultimo paragrafo è destinato, in ogni caso, alle osservazioni finali. 

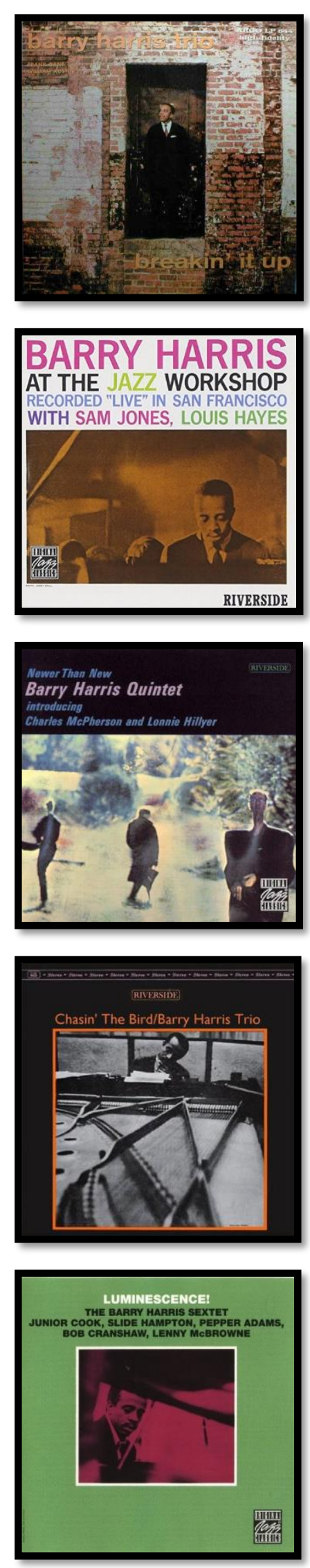

\section{Breakin' It Up}

(Argo, 1959)

Barry Harris - pianoforte

William Austin - contrabbasso

Frank Gant - batteria

\section{Barry Harris at the Jazz Workshop}

(Riverside, 1960)

Barry Harris - pianoforte

Sam Jones - contrabbasso

Louis Hayes - batteria

\section{Newer Than New}

(Riverside, 1961)

Lonnie Hillyer - tromba

Charles McPherson - sassofono contralto

Barry Harris - pianoforte

Ernie Farrow - contrabbasso

Clifford Jarvis - batteria

\section{Chasin' The Bird}

(Riverside, 1962)

Barry Harris - pianoforte

Bob Cranshaw - contrabbasso

Clifford Jarvis - batteria

\section{Luminescence!}

(Prestige, 1967)

Junior Cook - sassofono tenore

Pepper Adams - sassofono baritono

Slide Hampton - trombone

Barry Harris - pianoforte

Bob Cranshaw - contrabbasso

Lanny McBrowne - batteria 


\section{Improvvisare sugli Accordi di Prima Specie}

\subsection{Presentazione della "Frase Fondamentale"}

In questo capitolo verrà presentata un'utilissima metodologia improvvisativa Be-Bop da adoperarsi in presenza dell'accordo C7: al lettore è affidata la necessaria trasposizione nelle 12 tonalità.

L'unico background richiesto consiste essenzialmente in una ragionevole padronanza delle triadi. L'intera trattazione verrà condotta in termini di frasi e parole [3].

La "frase fondamentale" da memorizzare [18] [19] è mostrata nel sottostante Pentagramma (1):

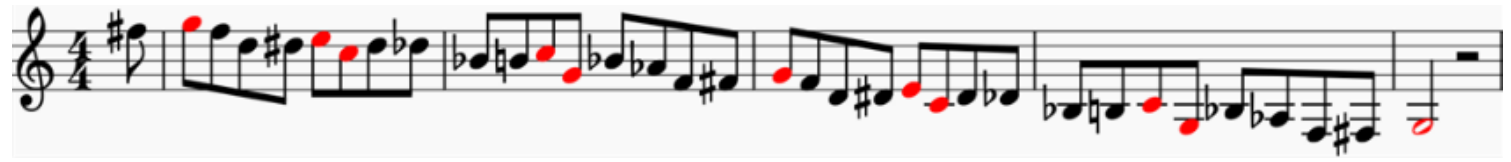

Nel Pentagramma (1), come del resto nella totalità dei pentagrammi inclusi nel capitolo (al netto di una singola eccezione), le note cordali pure (quelle costituenti la triade) sono rappresentate in rosso. La frase fondamentale, estesa intenzionalmente lungo due ottave adiacenti, consente di riprodurre, in una volta, l'intera gamma delle tensioni alterate dell'accordo (b13, \#11, \#9, b9).

\subsection{Focalizzare l'Attenzione sulla Triade Maggiore}

La frase fondamentale può essere evidentemente eseguita, considerando una semplice traslazione, partendo da ognuna delle note cordali pure.

È fondamentale sottolineare come, nel riprodurre tanto la frase fondamentale quanto le proposizioni derivate, l'attenzione debba essere costantemente focalizzata sulla triade maggiore (tanto in stato fondamentale quanto sotto forma di rivolto).

Dal Pentagramma (1), sfruttando la traslazione ed effettuando dei banali tagli, otteniamo immediatamente le tre utilissime frasi sottostanti:

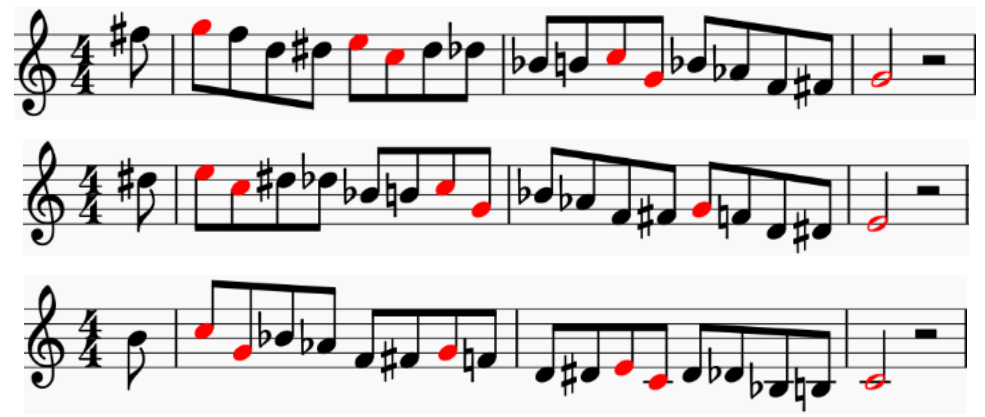

L'approccio ascendente (cromatico per definizione) che appare nelle anacrusi è da ritenersi opzionale. Sebbene l'argomento non venga in questa sede approfondito, è interessante notare come le frasi proposte nei Pentagrammi (2), (3) e (4), si prestino agevolmente a svariati spostamenti metrici. Una volta metabolizzate le frasi precedenti, è suggerita la pratica del sottostante esercizio, tanto banale quanto utile, basato sulla triade maggiore e gli approcci (cromatici) ascendenti: 


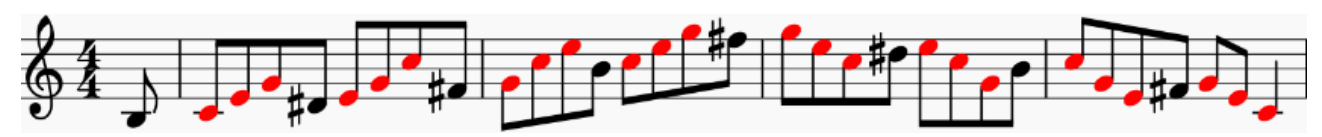

Dai Pentagrammi (2), (3) e (4) (il primo dei quali trasposto all'ottava inferiore), imponendo un banale spostamento metrico (una traslazione pari ad un intero movimento) ed avvalendoci dell'esercizio precedente, otteniamo le tre frasi sottostanti:

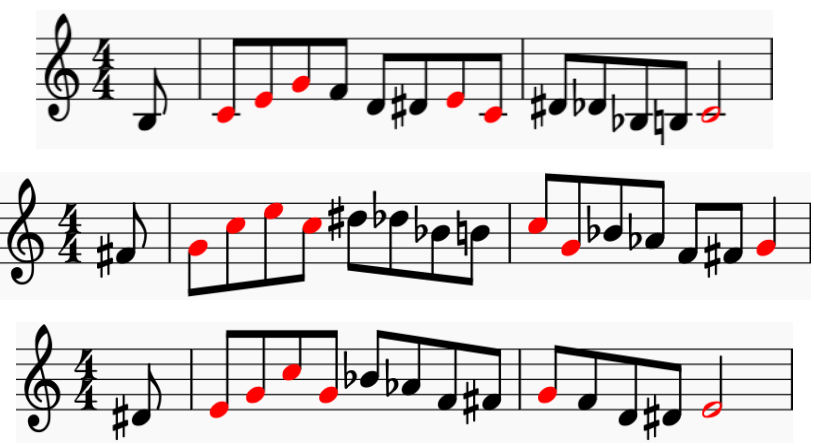

Siamo già in grado di combinare le semplici proposizioni finora ottenute, al fine di generarne altre assai più articolate. [3] [12] [13] [14] [17]

Ad esempio, dai Pentagrammi (6), (7) e (8) (il primo trasposto all'ottava superiore), possiamo dedurre la seguente frase:

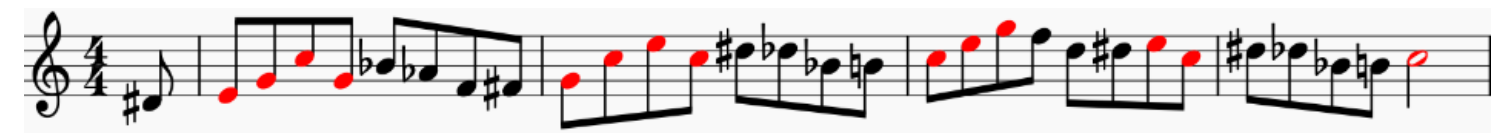

È ora suggerita la pratica del sottostante esercizio, basato sul concetto di approccio misto (richiamato varie volte nei capitoli precedenti):

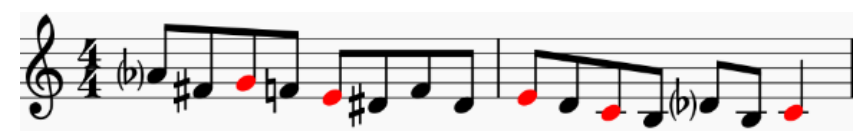

La frase precedente conferisce all'improvvisazione un "mood" decisamente "cromatico": tale effetto è amplificato dall'adozione degli accidenti opzionali riportati in parentesi.

In linea con quanto evidenziato all'inizio del paragrafo corrente, il musicista avrà cura, nell'eseguire l'esercizio proposto nel Pentagramma (10), di focalizzare la propria attenzione sulla triade maggiore. Naturalmente, è possibile proseguire nella combinazione delle frasi, ora con un "ingrediente" addizionale: ad esempio, dai Pentagrammi (3) e (10) (quest'ultimo trasposto all'ottava superiore), otteniamo immediatamente:

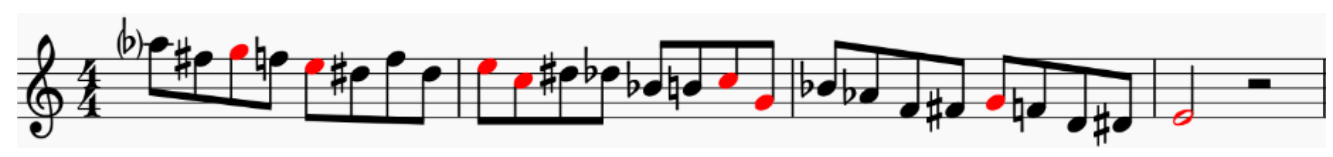

Consideriamo adesso la sottostante nuova parola, nulla più che un ponte cromatico bidirezionale (da percorrersi, dunque, ascendendo e discendendo) tra la quinta e la sesta:

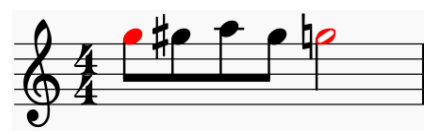

L'attenzione dell'esecutore, naturalmente, dev'essere focalizzata in via esclusiva sulla quinta.

Sfruttando la parola appena introdotta, è possibile dedurre un enorme quantitativo di nuove frasi. 
Ad esempio, dai Pentagrammi (2) e (12), il primo privato dell'anacrusi (procedura della quale ci serviremo all'occorrenza evitando, d'ora innanzi, di sottolinearlo) ed esteso alla terza, otteniamo:

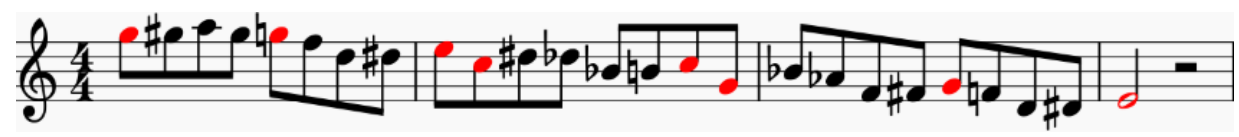

\subsection{Introduzione della Settima}

Consideriamo la seguente nuova parola, nient'altro che un semplice ponte cromatico discendente tra la settima minore (rappresentata in verde) e la quinta:

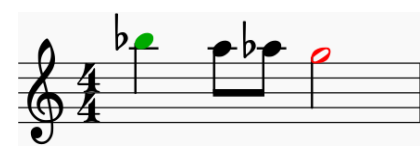

Dal Pentagramma (2), traslato ed esteso alla terza, e dal Pentagramma (14), otteniamo:

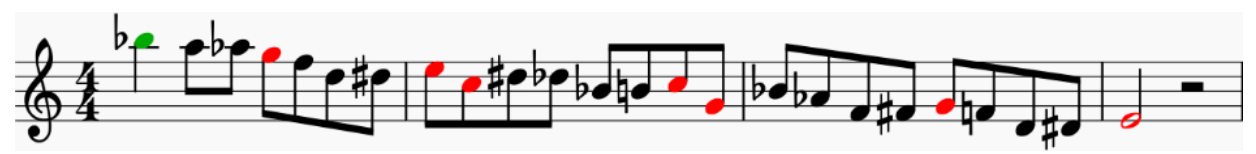

Dalla giustapposizione dei Pentagrammi (12) e (14), otteniamo la sottostante nuova parola:

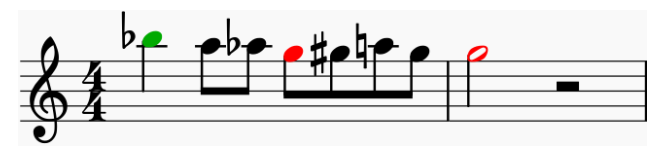

Sfruttando la parola appena ottenuta, dal Pentagramma (2), imponendo la solita estensione alla terza, deduciamo agevolmente la seguente frase:

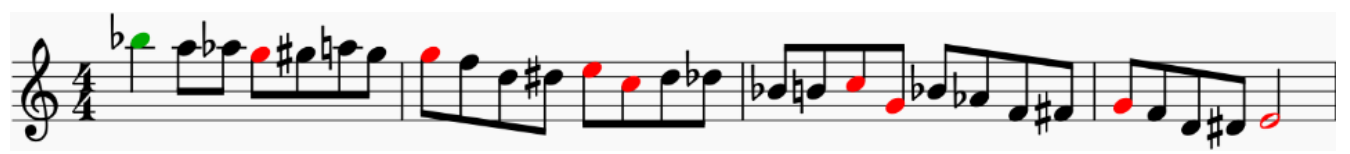

La frase presentata nel Pentagramma (2), della quale stiamo ripetutamente avvantaggiandoci, può essere modificata "tagliando corto". Una volta raggiunta la terza, in luogo della pedissequa riproduzione della frase, è contemplabile la costruzione di un ponte cromatico verso la tonica.

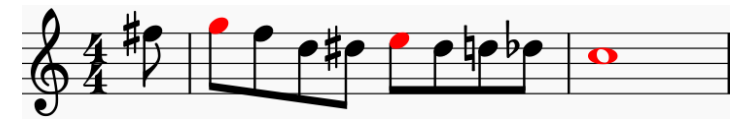

Vale la pena evidenziare come la frase precedente possa essere serenamente eseguita considerando l'accordo Fmaj7 in ultima battuta.

Dai Pentagrammi (12) e (18) otteniamo immediatamente:

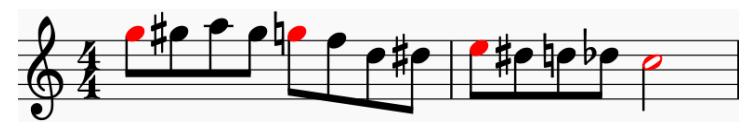

Recuperando la settima, dai Pentagrammi (14) e (18) deduciamo il seguente:

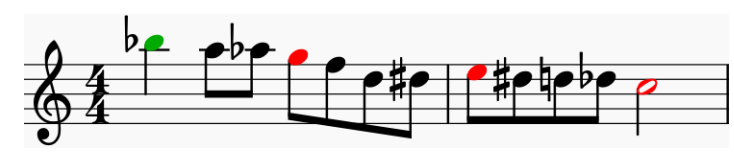


Similmente, dai Pentagrammi (16) e (18) otteniamo:

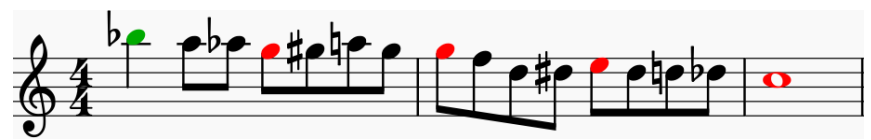

La frase appena dedotta può essere adoperata sulla progressione armonica $G-7|C 7|$ Fmaj7.

La frase nel Pentagramma (2) può essere ulteriormente modificata a mezzo d'una “deviazione". Una volta raggiunta la terza, anziché riprodurre la frase nella maniera in cui essa è stata inizialmente concepita, si devia lungo la scala Dominante Be-Bop (vedi Capitolo 1), sino a raggiungere la settima:

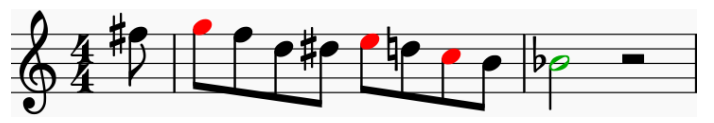

Dai Pentagrammi (12) e (22) abbiamo:

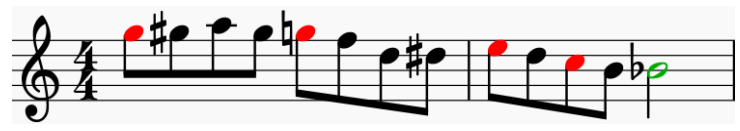

Dai Pentagrammi (14) e (22) abbiamo:

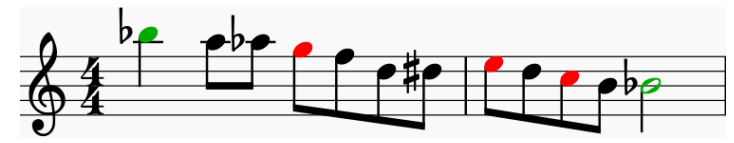

Dai Pentagrammi (14) e (23) abbiamo:

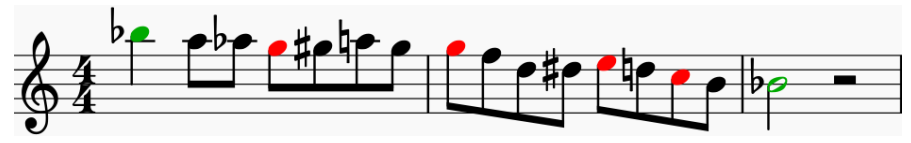

Estendendo la proposizione precedente lungo due ottave consecutive, otteniamo una nuova frase, in cui il ruolo della tonica è ora interpretato, per così dire, dalla settima minore:

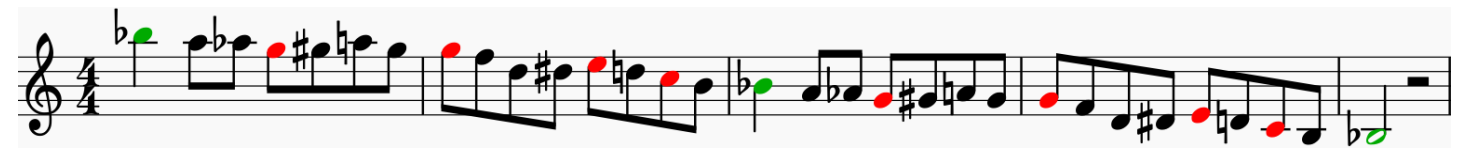

Dalla frase appena ottenuta, adoperando una procedura del tutto simile a quella sfruttata al fine di dedurre i Pentagrammi (2), (3) e (4), è possibile ricavare tre proposizioni semplici, omesse per brevità, che possono essere ovviamente modificate seguendo una metodologia che, a questo punto, dovrebbe risultare abbastanza chiara.

Evidentemente, siamo ora in grado di comporre un numero significativo di nuove frasi, così coprendo una larga fetta del cosiddetto "Vocabolario" Be-Bop. [3] [12] [13] [14] [17]

Ad esempio, sovrapponendo opportunamente i Pentagrammi (8), (22) e (25) (il primo trasposto all'ottava superiore, l'ultimo a quella inferiore ed arrestato alla terza), otteniamo agevolmente:

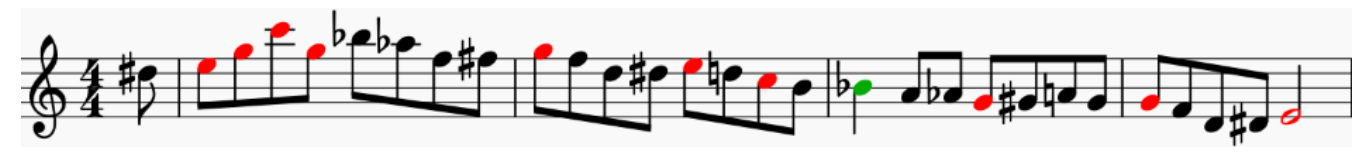

Ovviamente, ulteriori modificazioni, non importa quanto significative, consentono d'incrementare notevolmente il numero delle possibilità. A tal proposito, basti pensare che il ponte cromatico introdotto nel Pentagramma (18) può anche essere bidirezionale. 
Alla luce di quanto appena osservato, sfruttando la deviazione introdotta nel Pentagramma (22), possiamo agevolmente dedurre la sottostante utilissima frase:

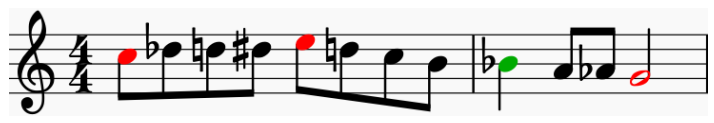

Dai Pentagrammi (9) e (28), agendo nel solito modo, abbiamo:

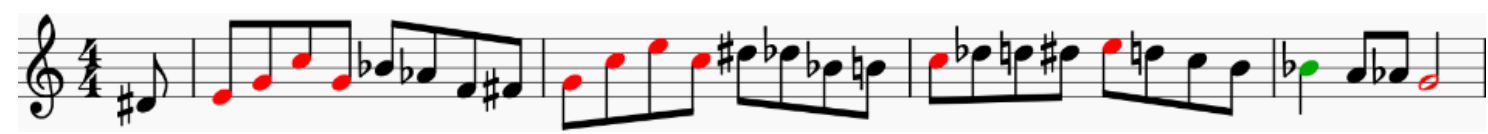

\subsection{Introduzione della Nona}

Il metodo brevemente presentato in questo capitolo dovrebbe oramai risultare sufficientemente chiaro: pertanto, per amore di sintesi, ometteremo la scrittura di singole parole nel paragrafo corrente. Anzitutto, è evidentemente possibile la costruzione di un semplice ponte cromatico discendente tra la nona e la settima (entrambe rappresentate in verde).

Sfruttando tale procedura, dal Pentagramma (25), trasposto all'ottava inferiore ed esteso alla quinta, possiamo dedurre la seguente frase:

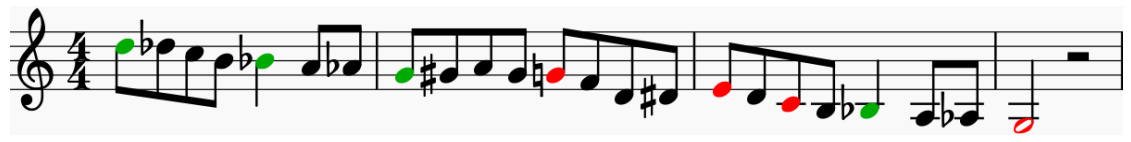

È naturalmente contemplabile la costruzione di ponti cromatici bidirezionali: il più semplice, ovviamente, è quello che coinvolge nona e decima (la terza all'ottava superiore).

Dai Pentagrammi (21) e (30), ad esempio, otteniamo:

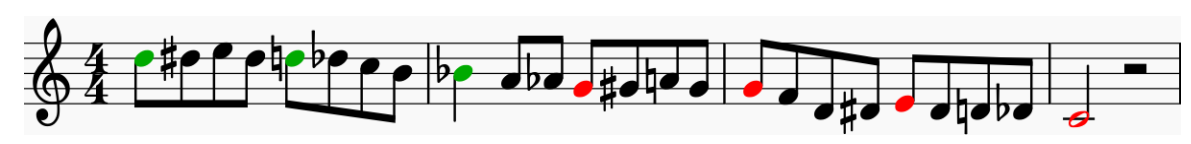

Il cromatismo bidirezionale di maggior interesse è quelle che coinvolge settima minore e nona.

Ad esempio, dal Pentagramma (30) possiamo immediatamente dedurre la sottostante frase:

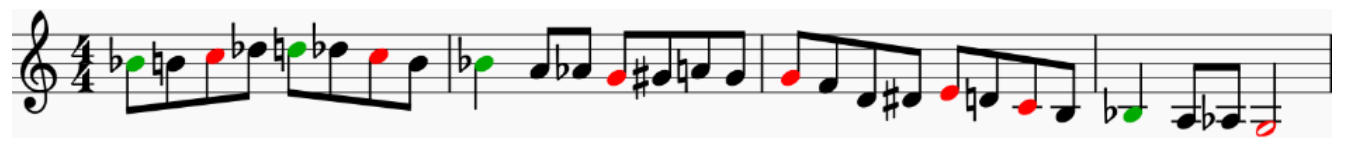

\subsection{Come "Arricchire" una Frase (cenni brevissimi)}

Non appena l'improvvisatore acquisisce sufficiente padronanza del metodo, può avere finalmente inizio una fase di "arricchimento". Sebbene la procedura non venga in questa sede affrontata, possiamo sinteticamente affermare come il modo più semplice per arricchire una frase consista nell'utilizzo di "ripetizioni" ed "oscillazioni diatoniche". [20] [21] [22] [23] Un semplice esempio di arricchimento, interamente basato sulla frase nel Pentagramma (30), è di seguito riportato:

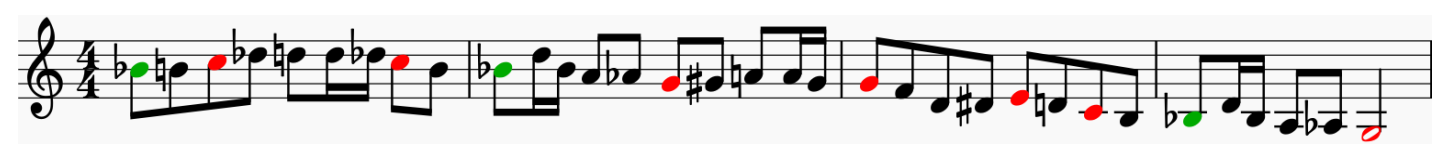




\subsection{Osservazioni Finali}

Nel Linguaggio Be-Bop, gli Accordi di Settima di Dominante sono spesso sostituiti con Accordi Diminuiti [2] [7] [8] [9] [24] costruiti su terza, quinta, settima (minore) e nona.

A tal proposito, si rivela utilissimo il seguente esercizio:

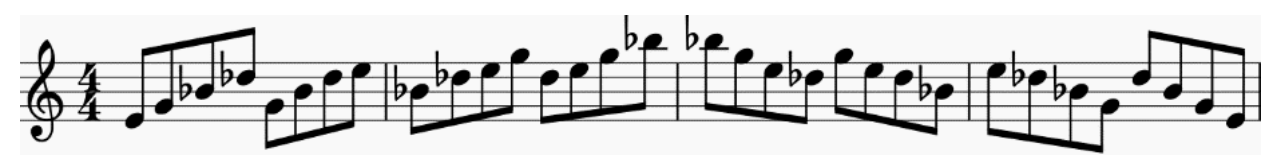

Sfruttando la sostituzione di cui sopra, è possibile modificare ulteriormente le frasi finora ottenute. Proponiamo, dunque, una coppia di utilissimi esempi, in cui la nona bemolle è intenzionalmente rappresentata in arancione.

Dal Pentagramma (25), trasposto all'ottava inferiore, otteniamo:

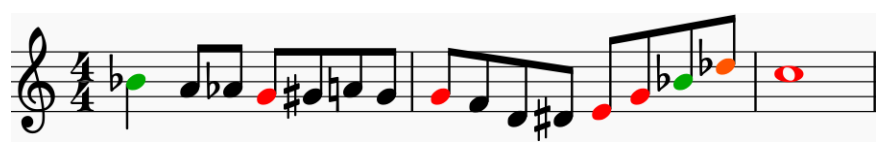

Dal Pentagramma (30), tagliato e privato del cromatismo bidirezionale sulla quinta, otteniamo:

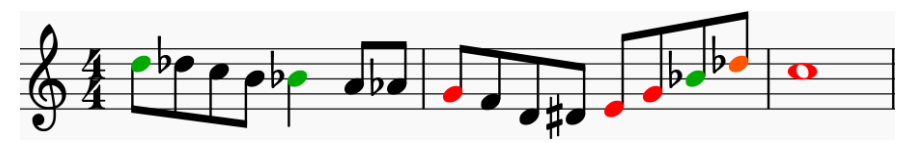

Le ultime due frasi possono essere evidentemente suonate sulla progressione $G-7|C 7|$ Fmaj7.

La cosa costituisce tutto fuorchè una coincidenza: al contrario, abbiamo finalmente l'opportunità di discutere qualitativamente il motivo per cui, nonostante l'introduzione di note non cordali (settima minore e nona), il metodo possa essere ancora considerato come basato esclusivamente sulle triadi. Nella prima battuta del Pentagramma (36), ad esempio, l'attenzione dell'improvvisatore dev'essere focalizzata non già sulla triade maggiore di $C$, bensì su quella minore di $G$. In certa misura, infatti, è lecito affermare come $C 7$ possa essere "trattato" come se fosse $G-7$ (i due accordi sono "parenti di secondo grado", siccome entrambi in relazione diatonica con $E-7 b 5)$. Di conseguenza, al netto di rare eccezioni, l'improvvisatore potrà serenamente suonare su $C 7$ la quasi totalità delle frasi adatte a $G-7$ (gli accordi di seconda saranno analizzati nel capitolo successivo), e vice versa, in tal modo ampliando considerevolmente il proprio linguaggio.

Alla luce del carattere squisitamente introduttivo del lavoro, è stata intenzionalmente evitata l'analisi delle metodologie d'impiego di alcune scale fondamentali (esafoniche, come quella a Toni Interi, eptafoniche, come la Super-Locria, octofoniche, come la Semitono-Tono), dei numerosi "pattern di pubblico dominio" (assimilabili ad espressioni idiomatiche ovvero a "riferimenti eruditi") [4] [25], nonché delle tipiche sostituzioni armoniche al tritono. [2] [7] [8] [9] 


\section{Improvvisare sugli Accordi di Seconda Specie}

\subsection{Presentazione della "Frase Fondamentale"}

In questo capitolo verrà presentata un'utilissima metodologia improvvisativa Be-Bop da adoperarsi in presenza dell'accordo $C-7$ : al lettore è affidata la necessaria trasposizione nelle 12 tonalità. L'unico background richiesto consiste essenzialmente in una ragionevole padronanza delle triadi. L'intera trattazione verrà condotta in termini di frasi e parole [3].

La "frase fondamentale" da memorizzare [26] [27] è mostrata nel sottostante Pentagramma (1):

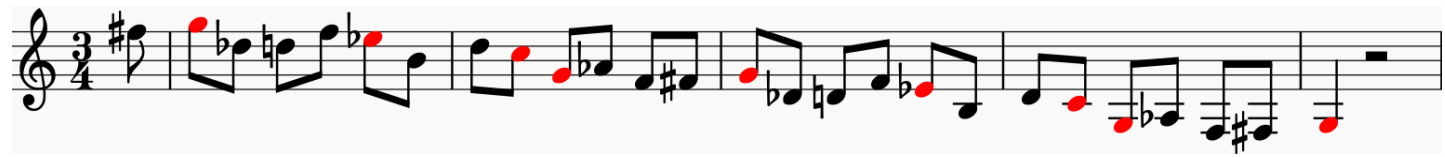

Nel Pentagramma (1), come del resto nella totalità dei pentagrammi inclusi nel presente capitolo (al netto di una singola eccezione), le note cordali pure (quelle costituenti la triade) sono rappresentate in rosso. La frase fondamentale è estesa intenzionalmente lungo due ottave adiacenti.

Nel Pentagramma (1), il tempo 3/4, adottato per mera convenienza, è da ritenersi squisitamente correlato alla "periodicità dispari" della particolare frase considerata: la frase fondamentale, infatti, può essere agevolmente riscritta in $6 / 4$, in tal modo enfatizzandone il carattere periodico.

\subsection{Focalizzare l'Attenzione sulla Triade Minore}

La frase fondamentale può essere evidentemente eseguita, considerando una semplice traslazione, partendo da ognuna delle note cordali. Naturalmente, nel riprodurre tanto la frase fondamentale quanto le proposizioni derivate, l'attenzione dev'essere costantemente focalizzata sulla triade minore (stato fondamentale e rivolti). Dal Pentagramma (1), sfruttando la traslazione ed effettuando dei semplici tagli, otteniamo immediatamente le tre utilissime frasi sottostanti:

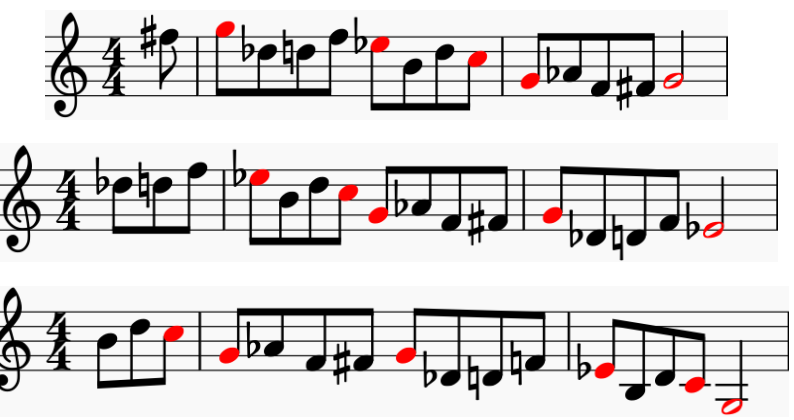

L'approccio ascendente (ovviamente cromatico) che appare nelle anacrusi è da ritenersi opzionale. Sebbene l'argomento non venga in questa sede approfondito, è interessante notare come le frasi proposte nei Pentagrammi (2), (3) e (4), si prestino agevolmente a svariati spostamenti metrici.

Una volta metabolizzate le frasi precedenti, è suggerita la pratica del sottostante esercizio, tanto banale quanto utile, basato sulla triade minore e gli approcci (cromatici) ascendenti: 


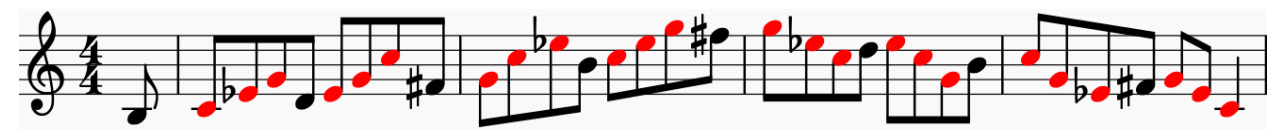

Imponendo un banale spostamento metrico, dall'esercizio precedente deduciamo il seguente:

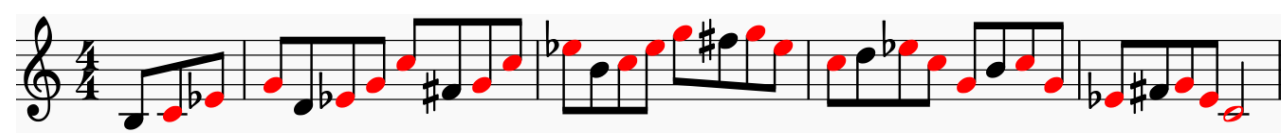

Dai Pentagrammi (2), (3) e (4) (quest'ultimo assoggettato a spostamento metrico), sfruttando l'esercizio proposto nel Pentagramma (6), otteniamo agevolmente le tre frasi sottostanti:

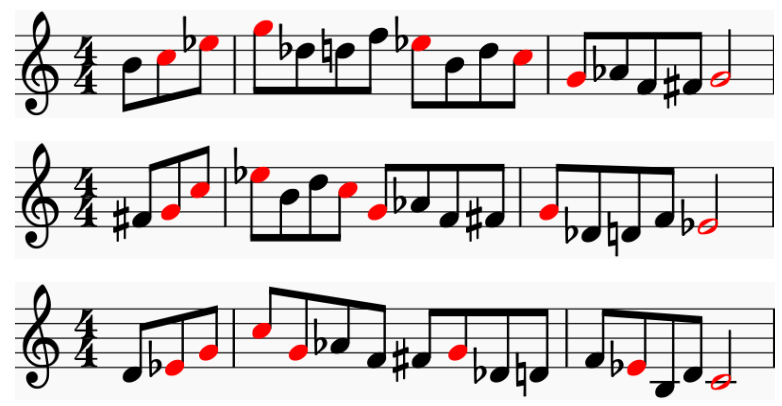

Siamo già in grado di combinare le semplici proposizioni finora ottenute, al fine di generarne altre assai più articolate [3] [12] [13] [14] [17]: a titolo d'esempio, dai Pentagrammi (6) e (8), quest'ultimo sviluppato proseguendo lungo la frase fondamentale presentata nel Pentagramma (1), otteniamo:

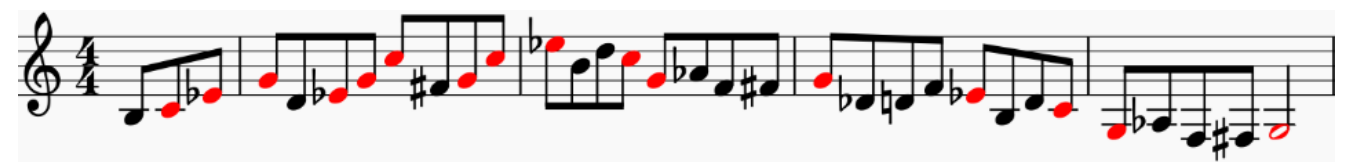

È ora suggerita la pratica del sottostante esercizio, basato sul concetto di approccio misto:

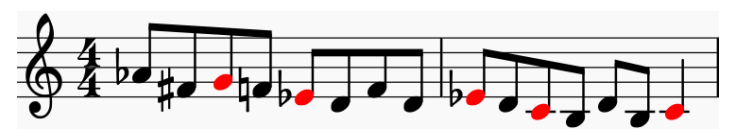

La frase precedente conferisce all'improvvisazione un "mood" decisamente "cromatico".

In linea con quanto evidenziato all'inizio del paragrafo corrente, il musicista avrà cura, nell'eseguire l'esercizio proposto nel Pentagramma (11), di focalizzare la propria attenzione sulla triade minore. Naturalmente, è possibile proseguire nella combinazione delle frasi, ora con un "ingrediente" addizionale: ad esempio, dal Pentagramma (3), privato dell'anacrusi ed esteso assecondando la frase fondamentale, e dal Pentagramma (11), trasposto all'ottava superiore, otteniamo immediatamente:

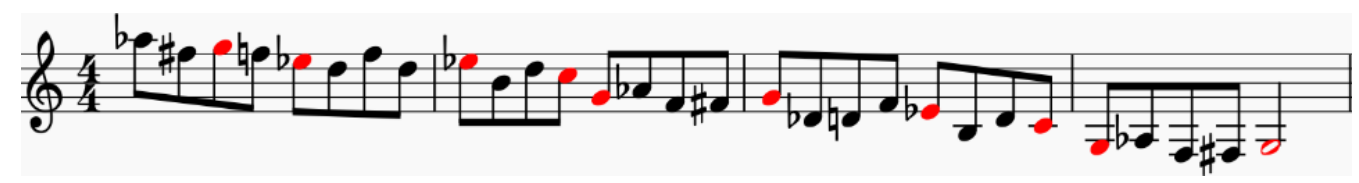

Sfruttando il concetto di approccio misto, richiamato nel Pentagramma (11), e considerando un moto ascendente, otteniamo la sottostante nuova parola (ovvero brevissima proposizione):

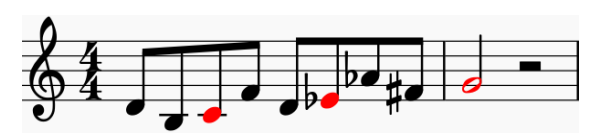

Adoperando la parola appena introdotta, è possibile dedurre un enorme quantitativo di nuove frasi. 
A titolo d'esempio, dal Pentagramma (2), trasposto all'ottava inferiore ed arrestato alla quinta, e dai Pentagrammi (11) e (13), possiamo dedurre la seguente frase articolata:

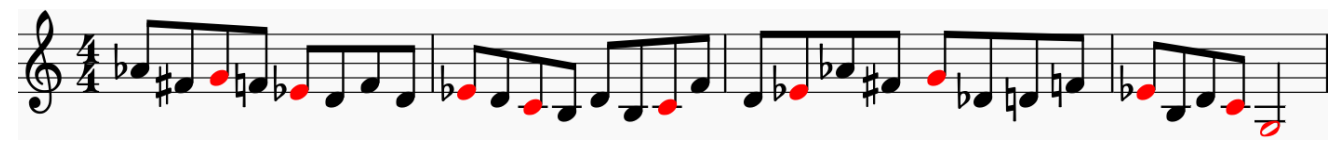

Consideriamo adesso la sottostante nuova parola, nulla più che un "ponte cromatico" bidirezionale (da percorrersi in entrambi versi, ascendendo e discendendo) tra la quinta e la sesta maggiore:

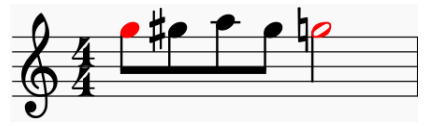

L'attenzione dell'esecutore, naturalmente, dev'essere focalizzata in via esclusiva sulla quinta.

Dai Pentagrammi (2) e (15) (il primo privato dell'anacrusi ed arrestato alla quinta) otteniamo:

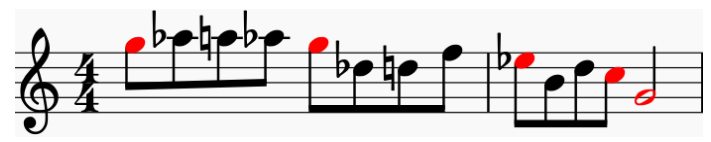

È fondamentale osservare come dai Pentagrammi (2) e (15) (il primo dei quali privato, ancora una volta, dell'anacrusi) sia inoltre possibile dedurre agevolmente un'utilissima versione alternativa, a "periodicità pari", della frase fondamentale presentata nel Pentagramma (1):

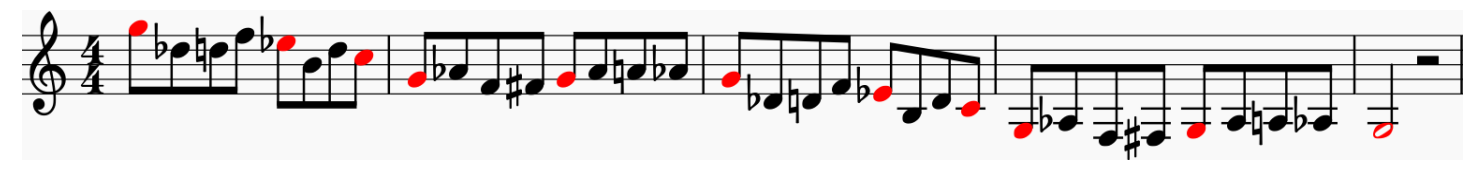

Naturalmente, seguendo una metodologia che, a questo punto, dovrebbe risultare sufficientemente chiara, dal Pentagramma (17) è possibile dedurre un notevole quantitativo di nuove proposizioni.

\subsection{Ponti Cromatici e Terze Diatoniche}

Consideriamo adesso un "lungo" ponte cromatico discendente tra la quinta e la tonica.

Il suddetto può essere immaginato come scaturito dalla giustapposizione di due ponti cromatici discendenti "corti" (il primo collega la quinta alla terza minore, il secondo quest'ultima alla tonica):

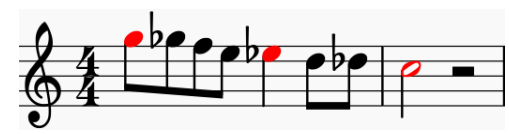

Ovviamente, i ponti corti possono anche essere adoperati tal quali.

Ad esempio, dal Pentagramma (2), arrestato una volta raggiunta la terza minore, possiamo agevolmente ricavare la seguente brevissima proposizione:

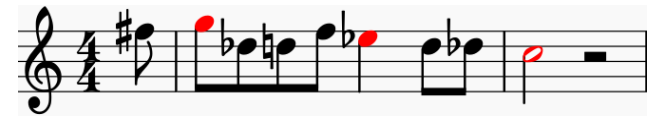

Similmente, dal Pentagramma (3), tagliato e considerato privo dell'anacrusi, otteniamo:

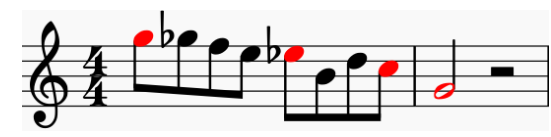


Dai Pentagrammi (15) e (18) ricaviamo istantaneamente la seguente frase:

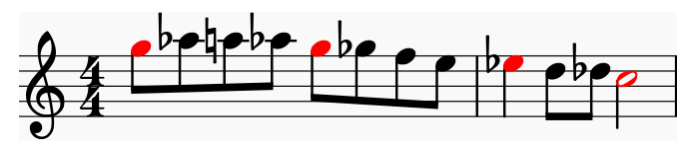

Analogamente, dai Pentagrammi (15) e (20) otteniamo:

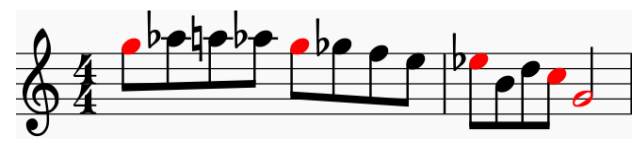

Consideriamo adesso la sottostante nuova parola:

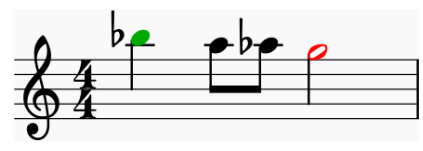

Molto evidentemente, la parola appena introdotta rappresenta nulla più che un ponte cromatico discendente tra la settima minore (rappresentata in verde) e la quinta.

Dai Pentagrammi (18) e (23) deduciamo la seguente frase:

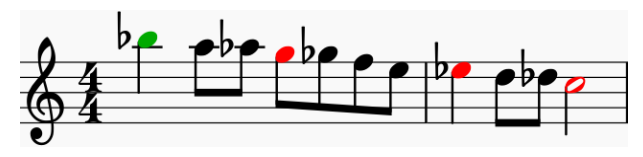

Similmente, dai Pentagrammi (20) e (23) otteniamo:

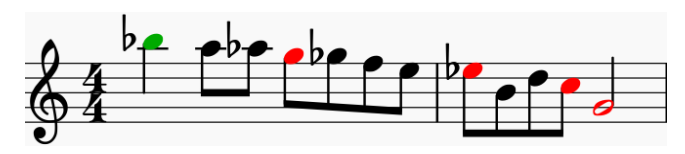

Dai Pentagrammi (21) e (23), il primo dei quali arretato alla terza, otteniamo:

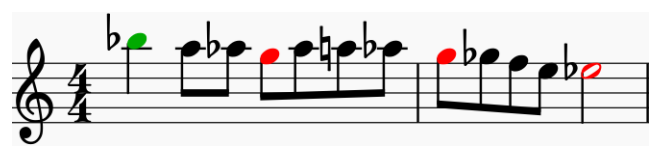

Seguendo la linea di ragionamento sinora sfruttata, è possibile introdurre tutte le ulteriori "tensioni ammissibili" (nona, undicesima, tredicesima). Assai evidentemente, le suddette sono tra di loro collegabili adoperando una banale scala cromatica.

Il Pentagramma (27) schematizza la modalità di costruzione di ponti cromatici discendenti, finalizzati a stabilire collegamenti tra le tensioni ammissibili adiacenti (tra le varie terze diatoniche), focalizzando l'attenzione su tre triadi maggiori "fondamentali".

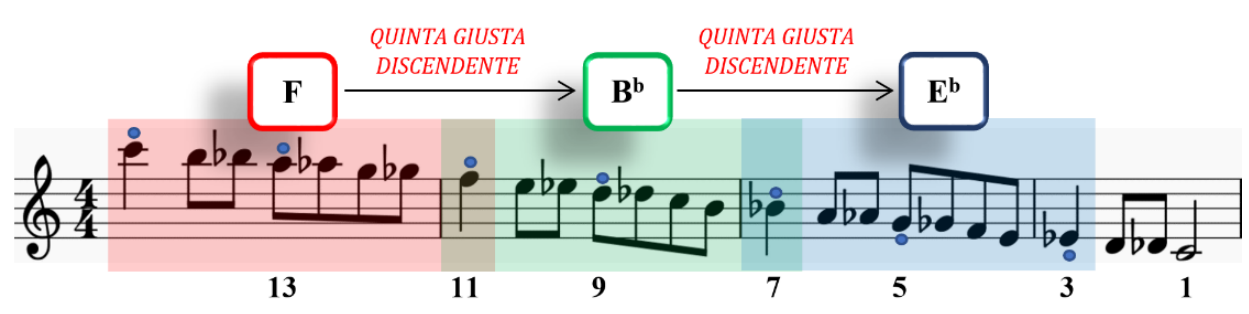




\subsection{Tonicizzazione}

L'accordo di settima di Seconda Specie può essere assoggettato a "tonicizzazione". [2] [7] [8] [9] La suddetta procedura si rende praticamente indispensabile qualora l'accordo resti invariato per un certo numero di battute (basti pensare a strutture come quella di "So What").

Ad esempio, $C-7$ può essere seguito e/o preceduto da $G 7$, quest'ultimo spesso considerato provvisto della nona bemolle, al fine di legittimare la cosiddetta "sostituzione diminuita". [2] [7] [8] [9] [24] Uno dei punti di forza del metodo consiste nella completa adattabilità delle frasi dedotte (nonché di quelle ulteriormente deducibili) agli accordi di seconda specie tonicizzati.

È agevole verificare come la frase nel Pentagramma (2), ad esempio, possa essere serenamente adoperata in presenza di qualsivoglia tipologia di tonicizzazione costruita su $C-7$ :

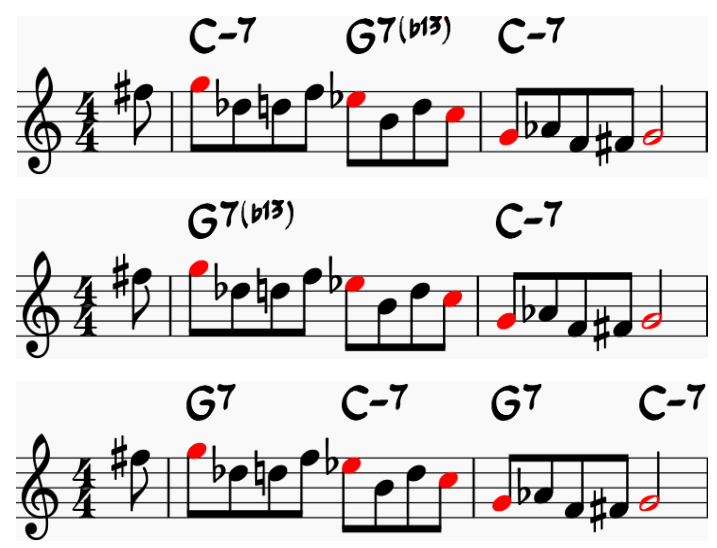

A titolo d'ulteriore esempio, possiamo considerare il Pentagramma (24):

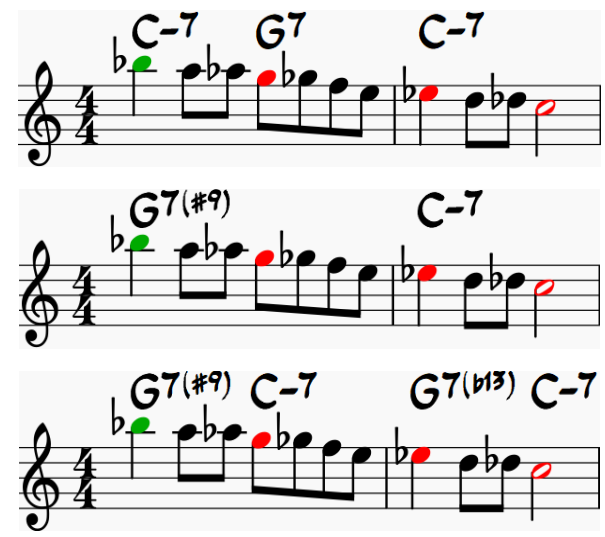

Naturalmente, l'idoneità delle frasi può essere localmente verificata tenendo ben presente il fatto che, com'è noto, gli accordi di settima di dominante possono essere deliberatamente alterati.

Nei Pentagrammi (28), (29) e (33) (riferendosi esclusivamente, per quanto concerne 1'ultimo, all'ultima battuta), Eb rappresenta la tredicesima bemolle di $G$; nei Pentagrammi (32) e (33) (riferendosi esclusivamente, per quanto concerne l'ultimo, alla prima battuta) $B b$ (siccome enarmonico con $A^{\#}$ ) rappresenta la nona diesis di $G$.

\subsection{Accordi Parenti}

\subsubsection{Accordo Parente di Prima Specie}

Nel linguaggio Be-Bop, al netto d'eccezioni relativamente rare, un accordo di settima di seconda specie può essere tranquillamente associato al Modo Dorico. [2] In altre parole, a prescindere dal 
ruolo rivestito nella particolare progressione armonica e nonostante eventuali tonicizzazioni, un accordo di settima di seconda specie può sempre essere immaginato come un Secondo Grado di un accordo di quarta specie (Modo Ionico).

Nel nostro caso, conseguentemente, $C-7$ può essere considerato "parente" (di "secondo grado") di $F 7$, siccome entrambi scaturiscono dall'armonizzazione di Bb Ionico. [5] [6] [7]

A tal proposito, sfruttando quanto dedotto nel capitolo precedente, consideriamo una delle frasi fondamentali adatte a $F 7$. La frase in questione è riportata nel sottostante Pentagramma (34), con le note cordali pure (quelle costituenti la triade maggiore di $F$ ) in rosso, a la settima minore (rispetto a $F$ ) in verde.

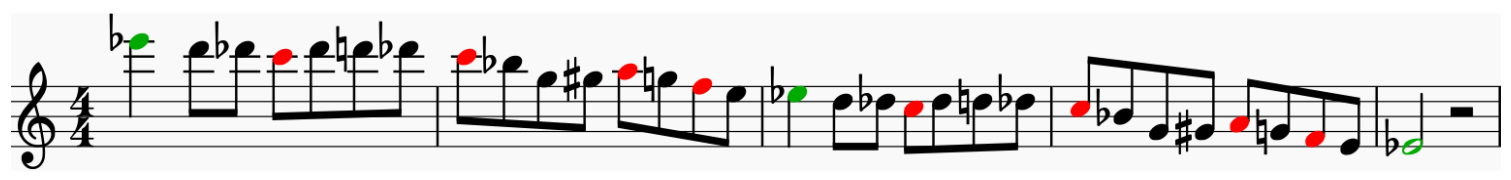

Nel Pentagramma (35) è riportata nuovamente la frase richiamata nel Pentagramma (34): in questo caso, tuttavia, le note cordali pure, rappresentate in rosso, e la settima, rappresentata in verde, si riferiscono nuovamente a $C-7$.

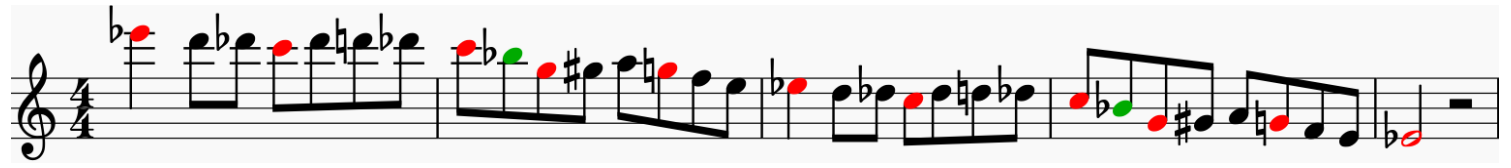

È semplice verificare come la frase riportata nel Pentagramma (35) sia perfettamente adatta a $C-7$. Seguendo la solita procedura, è chiaramente possibile la deduzione d'un numero significativo di ulteriori frasi, omesse per brevità, per di più modificabili e combinabili con quelle sinora ottenute. In aggiunta, possiamo affermare come, in virtù della relazione armonica discussa nel paragrafo corrente, tutte le frasi adatte a $C-7$ lo siano anche per $F 7$, sebbene a condizione d'adottare alcune "precauzioni": alcune di esse, in fatti, "funzionano" meglio quando l'accordo $F 7$ è provvisto nella nona diesis, altre sono da preferirsi, invece, quando l'accordo è sospeso, e così via.

\subsubsection{Accordo Parente di Terza Specie}

Alla luce delle relazioni armoniche brevemente discusse nel sotto-paragrafo precedente, $C-7$ può anche essere considerato "parente" di $A-7 b 5$. Più precisamente, $A-7 b 5$ (modo Locrio) è il "parente in comune" tra $C-7$ (modo Dorico, una terza minore ascendente) e $F 7$ (modo Misolidio, una terza maggiore discendente). [5] [6] [7]

A tal proposito, consideriamo nuovamente la frase adatta a $F 7$ riportata nel Pentagramma (34).

Nel Pentagramma (35) abbiamo riscritto la suddetta frase "in funzione" di $C-7$. Adesso, riscriviamo esattamente la stessa frase "in funzione" di $A-7 b 5$, riportando in rosso le note cordali pure (stavolta quelle che costituiscono la triade diminuita di $A$ ), e in verde la settima minore (stavolta rispetto ad $A$ ):

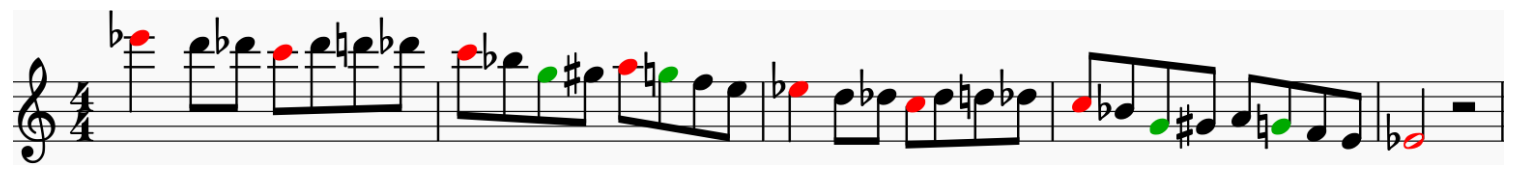

Anche in questo caso, è agevole verificare come la frase riportata nel Pentagramma (36) sia perfettamente adatta a $A-7 b 5$. Essa può inoltre generare, agendo nel solito modo, tre proposizioni (una per ognuna delle note cordali pure), che possono essere modificate e combinate nel rispetto della procedura in questa sede presentata, così da creare un "vocabolario" adatto per gli accordi di settima 
di Terza Specie. Il numero delle frasi idonee agli accordi di terza specie può essere ulteriormente incrementato sfruttando la relazione armonica discussa nel sotto-paragrafo corrente e in quello precedente. Per fornire un esempio, basato sul caso specifico brevemente esaminato in questa sede, è agevole verificare come molte delle frasi adatte a $C-7$ e $F 7$ possano essere suonate su $A-7 b 5$.

\subsection{Osservazioni Finali}

Tutte le frasi dedotte in questo capitolo, al netto di possibili "arricchimenti" (si veda il capitolo precedente) e combinazioni con "pattern di pubblico dominio" [4] [25], possono essere agevolmente rinvenute esaminando le trascrizioni di alcuni assoli notevoli [21] [22] [23] [28].

Al lettore dovrebbe apparire oramai piuttosto chiaro come la peculiarità del metodo in questa sezione presentato risieda, fondamentalmente, nel fatto che l'attenzione dell'improvvisatore, cui è richiesto un background davvero minimale, è quasi esclusivamente focalizzata sulle triadi: di base, non occorre altro per produrre un fraseggio Be-Bop anche piuttosto articolato. Naturalmente, la conoscenza dell'armonia e la capacità di padroneggiare scale fondamentali e cliché consente al musicista, al di là di ogni ragionevole dubbio, di arricchire ulteriormente il proprio linguaggio nonché, fattore tutt'altro che secondario, d'improvvisare con una ragionevole consapevolezza. [29] 

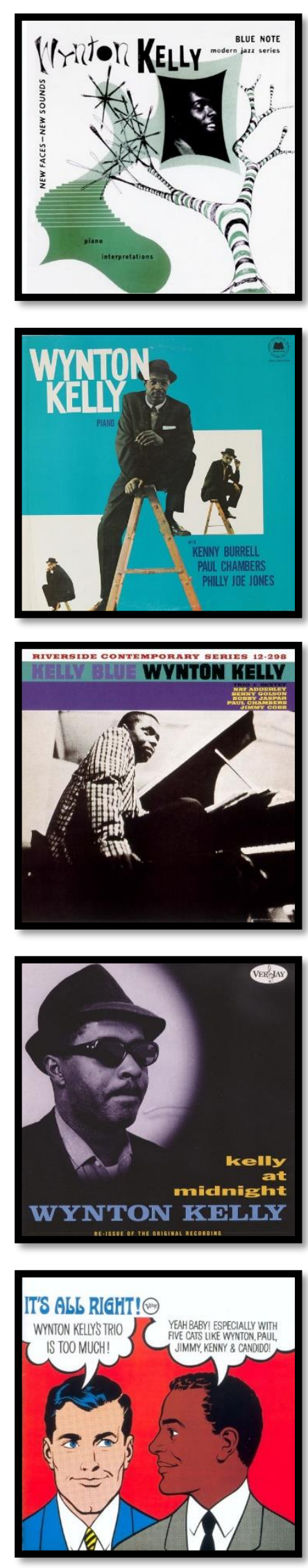

\section{Piano Interpretations}

(Blue Note, 1951)

Wynton Kelly - pianoforte, celeste

Oscar Pettiford / Franklin Skeete - contrabasso

Lee Abrams - batteria, conga

\section{Piano}

(Riverside, 1958)

Kenny Burrell - chitarra

Wynton Kelly - pianoforte

Paul Chambers - contrabbasso

Philly Joe Jones - batteria

\section{Kelly Blue}

(Riverside, 1959)

Nat Adderley - cornetta

Bobby Jaspar - flauti

Benny Golson - sassofono tenore

Wynton Kelly - pianoforte

Paul Chambers - contrabbasso

Jimmy Cobb - batteria

\section{Kelly at Midnight}

(Vee-Jay, 1960)

Wynton Kelly - pianoforte

Paul Chambers - contrabbasso

Philly Joe Jones - batteria

\section{It's All Right!}

(Verve, 1964)

Kenny Burrell - chitarra

Wynton Kelly - pianoforte

Paul Chambers - contrabbasso

Jimmy Cobb - batteria

Candido Camero - conga

The Tommy Rey Caribe Steel Band 


\section{Improvvisare sugli Accordi di Quarta Specie}

\subsection{Presentazione della "Frase Fondamentale"}

In questo capitolo verrà presentata un'utilissima metodologia improvvisativa Be-Bop da adoperarsi in presenza dell'accordo Cmaj7: al lettore è affidata la necessaria trasposizione nelle 12 tonalità. L'unico background richiesto consiste essenzialmente in una ragionevole padronanza delle triadi. L'intera trattazione verrà condotta in termini di frasi e parole [3].

La "frase fondamentale" da memorizzare [30] [31] è mostrata nel sottostante Pentagramma (1):

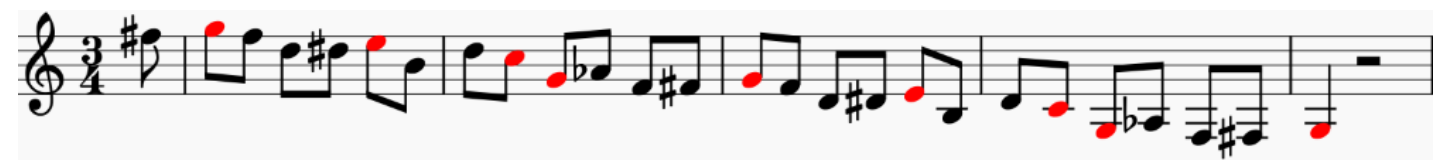

Nel Pentagramma (1), come del resto nella totalità dei pentagrammi inclusi nel presente capitolo, le note cordali pure (quelle costituenti la triade) sono rappresentate in rosso. La frase fondamentale è estesa intenzionalmente lungo due ottave adiacenti.

Nel Pentagramma (1), il tempo 3/4, adottato per mera convenienza, è da ritenersi squisitamente correlato alla "periodicità dispari" della particolare frase considerata: la frase fondamentale, infatti, può essere agevolmente riscritta in $6 / 4$, in tal modo enfatizzandone il carattere periodico.

\subsection{Focalizzare l'Attenzione sulla Triade Maggiore}

La frase fondamentale può essere evidentemente eseguita, considerando una semplice traslazione, partendo da ognuna delle note cordali. È fondamentale sottolineare come, nel riprodurre tanto la frase fondamentale quanto le proposizioni derivate, l'attenzione debba essere costantemente focalizzata sulla triade maggiore (stato fondamentale e rivolti). Dal Pentagramma (1), sfruttando la traslazione ed effettuando dei semplici tagli, otteniamo immediatamente le tre utilissime frasi sottostanti:
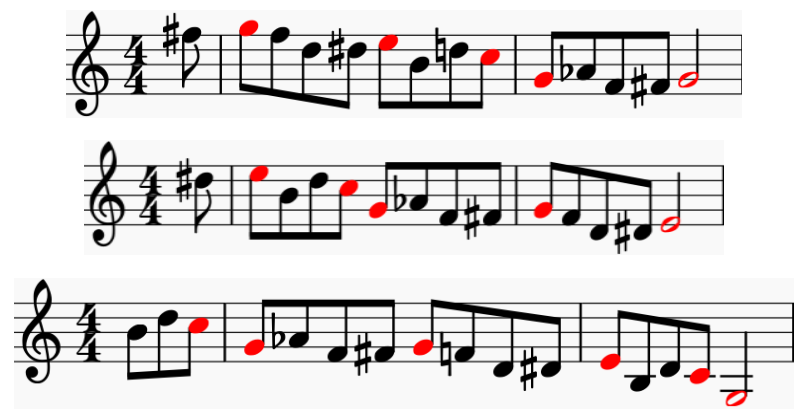

Le anacrusi sono da ritenersi opzionali.

Sebbene l'argomento non venga in questa sede approfondito, è interessante notare come le frasi proposte nei Pentagrammi (2), (3) e (4), si prestino agevolmente a svariati spostamenti metrici.

Una volta metabolizzate le frasi precedenti, è suggerita la pratica del sottostante esercizio, tanto banale quanto utile, basato sulla triade maggiore e gli approcci (cromatici) ascendenti: 


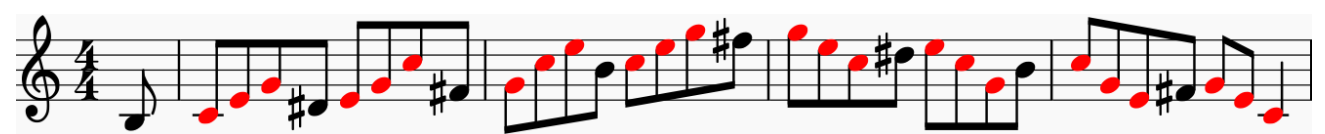

Imponendo un banale spostamento metrico, dall'esercizio precedente deduciamo il seguente:

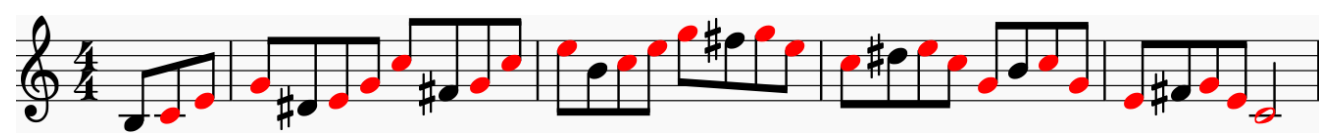

Dai Pentagrammi (2), (3) e (4) (quest'ultimo assoggettato a spostamento metrico e trasposto all'ottava superiore), sfruttando il Pentagramma (6), otteniamo agevolmente le tre frasi sottostanti:

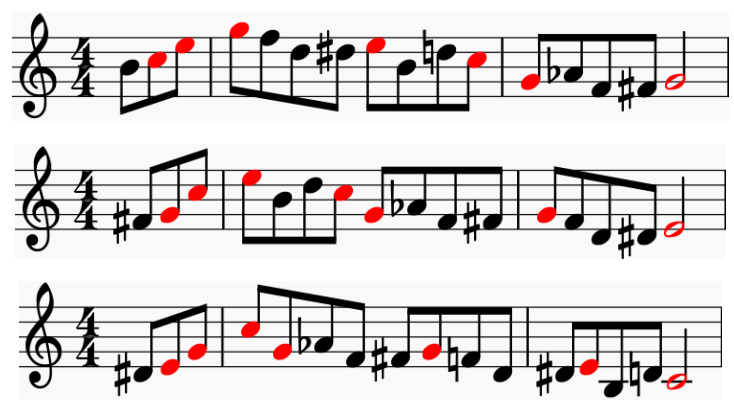

Siamo già in grado di combinare le semplici proposizioni finora ottenute, al fine di generarne altre assai più articolate. [3] [12] [13] [14] [17] Ad esempio, dai Pentagrammi (6) e (8), quest'ultimo sviluppato proseguendo come mostrato nel Pentagramma (1), otteniamo:

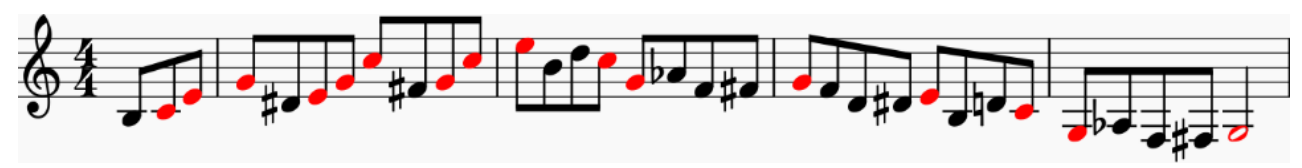

È ora suggerita la pratica del sottostante esercizio, basato sul concetto di approccio misto (richiamato varie volte nei capitoli precedenti):

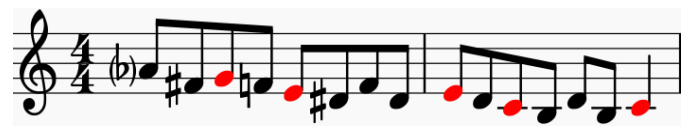

La frase precedente conferisce all'improvvisazione un "mood" decisamente "cromatico".

In linea con quanto evidenziato all'inizio del paragrafo corrente, il musicista avrà cura, nell'eseguire l'esercizio proposto nel Pentagramma (11), di focalizzare la propria attenzione sulla triade minore. Naturalmente, è possibile proseguire nella combinazione delle frasi, ora con un "ingrediente" addizionale: ad esempio, dal Pentagramma (3), esteso assecondando la frase fondamentale presentata nel Pentagramma (1), e dal Pentagramma (11), trasposto all'ottava superiore, otteniamo:

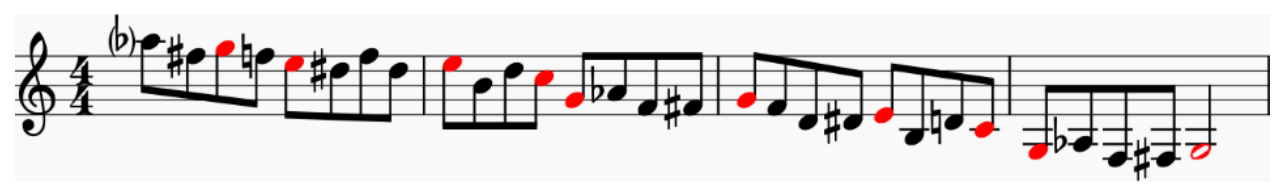

Sfruttando il concetto di approccio misto, richiamato nel Pentagramma (11), e considerando un moto ascendente, otteniamo la sottostante nuova parola (ovvero brevissima proposizione):

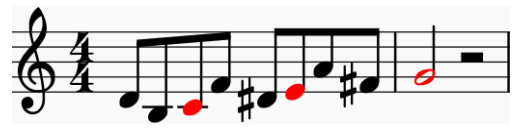


Adoperando la parola appena introdotta, è possibile dedurre un enorme quantitativo di nuove frasi. A titolo d'esempio, dal Pentagramma (2), trasposto all'ottava inferiore ed arrestato alla quinta, e dai Pentagrammi (11) e (13), possiamo dedurre la seguente frase articolata:

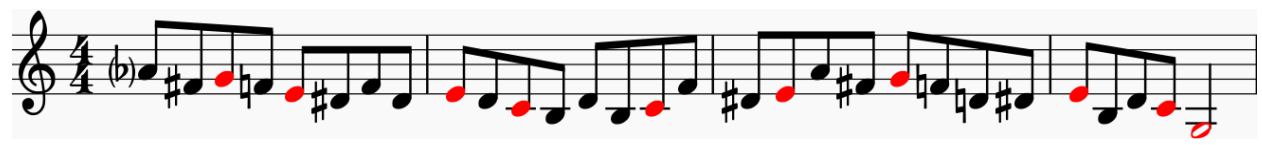

Consideriamo adesso la sottostante nuova parola, nulla più che un "ponte cromatico" bidirezionale (da percorrersi, dunque, in entrambi versi, ascendendo e discendendo) tra la quinta e la sesta:

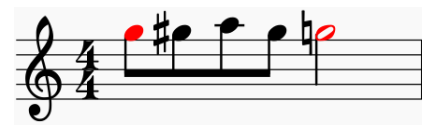

L'attenzione dell'esecutore, naturalmente, dev'essere focalizzata in via esclusiva sulla quinta. Dai Pentagrammi (2) e (15) (il primo privato dell'anacrusi ed arrestato alla quinta) otteniamo:

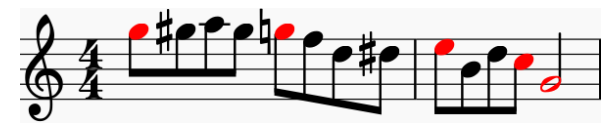

È fondamentale osservare come dai Pentagrammi (2) e (15) (il primo dei quali privato, ancora una volta, dell'anacrusi) sia inoltre possibile dedurre agevolmente un'utilissima versione alternativa, a "periodicità pari", della frase fondamentale presentata nel Pentagramma (1):

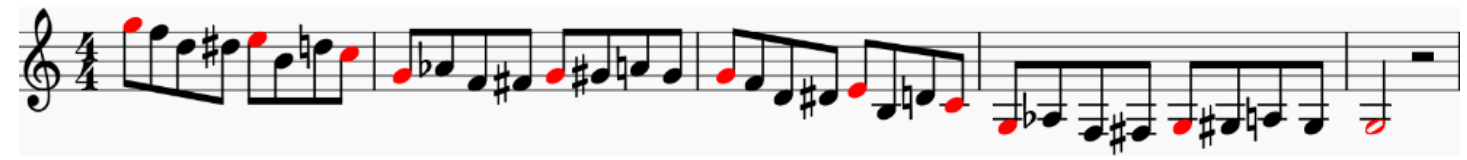

Naturalmente, seguendo una metodologia che, a questo punto, dovrebbe risultare sufficientemente chiara, dal Pentagramma (17) è possibile dedurre un notevole quantitativo di nuove proposizioni.

\subsection{Introduzione della Settima}

Consideriamo la seguente nuova parola, nient'altro che un semplice ponte cromatico discendente tra la settima maggiore (rappresentata in verde) e la quinta:

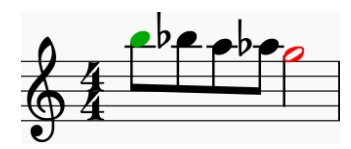

Dai Pentagrammi (2) e (18), il primo dei quali privato dell'anacrusi ed arrestato alla quinta, abbiamo:

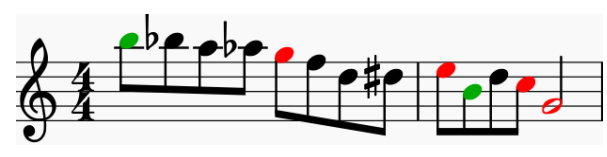

Dal Pentagramma (2) (privato dell'anacrusi ed arrestato alla quinta), adoperando una versione bidirezionale del ponte cromatico introdotto nel Pentagramma (18), è possibile dedurre agevolmente un'ulteriore frase fondamentale, che riportiamo estesa lungo due ottave consecutive, dalla quale sono ricavabili tre ulteriori proposizioni, omesse per brevità, a loro volta assoggettabili, adottando la metodologia finora esposta, a svariate modificazioni. 


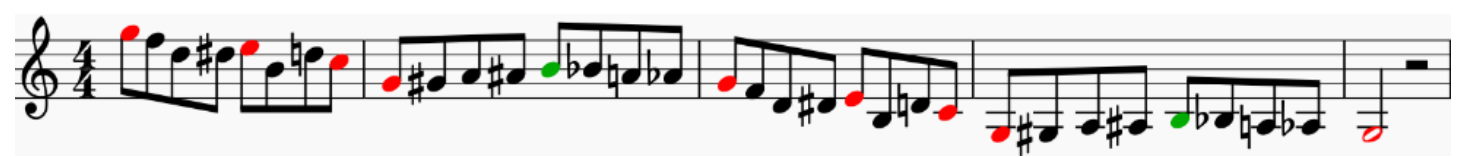

Dal Pentagramma (14), effettuando un banale taglio e sfruttando il ponte cromatico bidirezionale tra quinta e settima, otteniamo immediatamente:

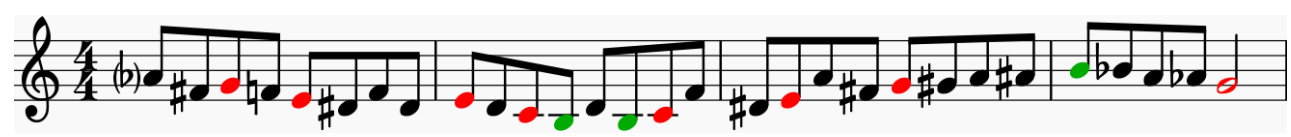

Dal Pentagramma (13), adoperando la frase presentata nel Pentagramma (20), abbiamo:

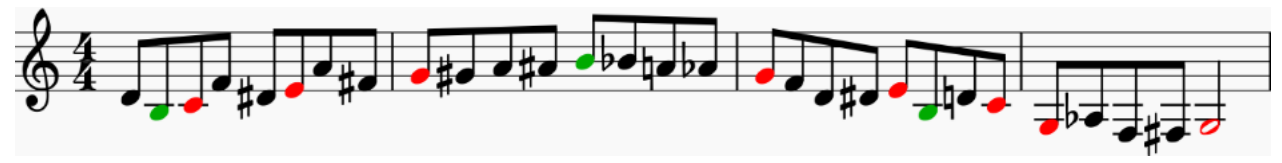

Dal Pentagramma (16), sfruttando nuovamente la frase proposta nel Pentagramma (20), otteniamo:

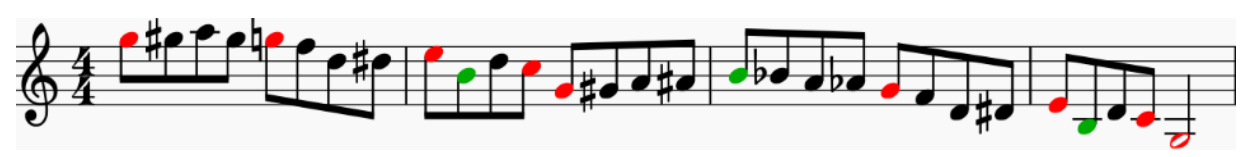

\subsection{Introduzione della Nona}

Consideriamo adesso le seguenti due parole, tra di loro estremamente simili, qualificabili come banali ponti cromatici discendenti tra la nona e la settima maggiore (entrambe rappresentate in verde):

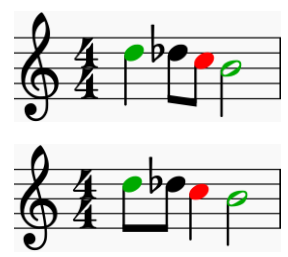

Ovviamente, la seconda parola pone un'enfasi maggiore sulla tonica.

Sfruttando il Pentagramma (18), dai Pentagrammi (24) e (25) possiamo dedurre, ordinatamente, le due seguenti brevi proposizioni:

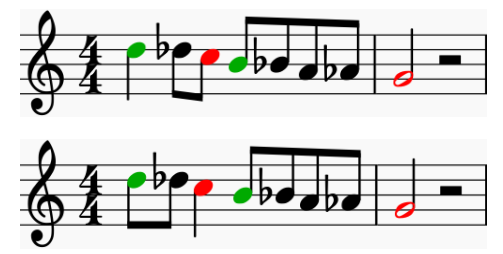

A questo punto, è assai agevole la deduzione d'un considerevole quantitativo di nuove frasi, la cui complessità dipende, sostanzialmente, dalle capacità maturate nell'effettuare tagli e combinazioni. Ad esempio, dalla sovrapposizione dei Pentagrammi (19) e (27), il primo dei quali trasposto all'ottava inferiore ed arrestato alla terza, otteniamo immediatamente la seguente breve proposizione:

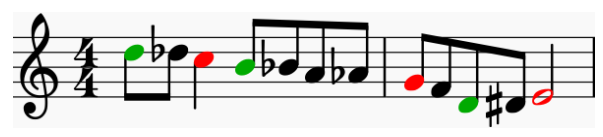


Tanto per fornire un ulteriore esempio, dai Pentagrammi (17) e (28), sfruttando il ponte cromatico bidirezionale tra quinta e settima, otteniamo agevolmente:

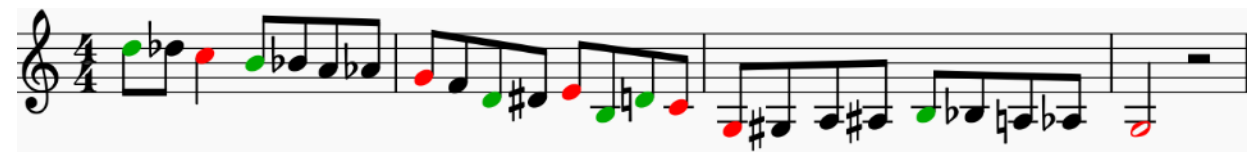

Vale la pena evidenziare come la frase proposta nel Pentagramma (28) possa essere leggermente modificata [20] [21] [22] [23] [24] nella seguente maniera:

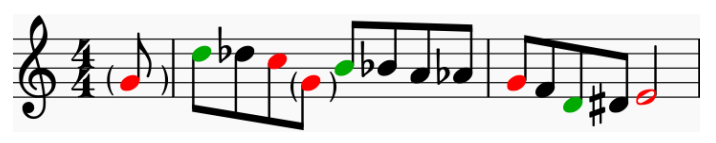

Tenendo a mente il fatto che, nel rispetto delle cosiddette sostituzioni diatoniche [7] [8] [9], ogni accordo di quarta specie può essere rimpiazzato da uno di seconda distante una terza maggiore ascendente (ovvero minore discendente), possiamo considerare la seguente nuova parola:

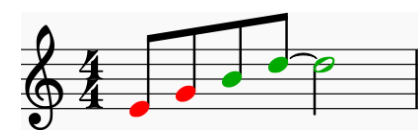

Dai Pentagrammi (30) e (31) deduciamo il seguente utilissimo "Be-Bop Loop":

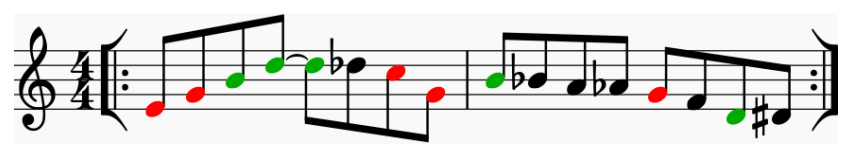

Dal loop appena presentato nel Pentagramma (32), è possibile ottenere istantaneamente, a mezzo di banali traslazioni, le seguenti quattro frasi:

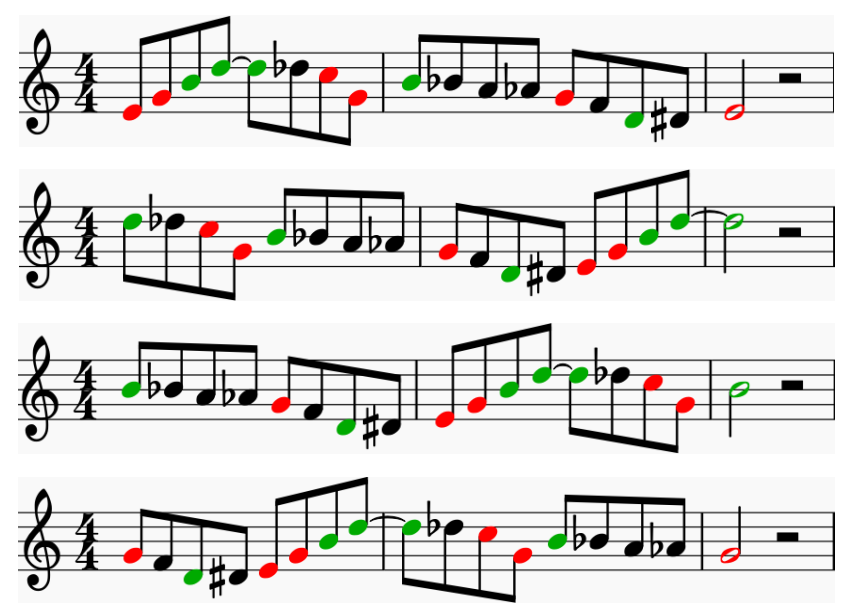

\subsection{Tonicizzazione e Turnaround}

L'Accordo di Settima di Quarta Specie può essere assoggettato a "Tonicizzazione" [2] [7] [8] [9]. La suddetta procedura si rende praticamente indispensabile qualora l'accordo resti sostanzialmente invariato per un certo numero di battute. Ad esempio, Cmaj7 può essere seguito e/o preceduto da $G 7$, quest'ultimo spesso considerato, onde legittimare la cosiddetta "sostituzione diminuita", provvisto della nona bemolle. [2] [7] [8] [9] [24] 
Uno dei punti di forza del metodo consiste nel fatto che la totalità delle frasi dedotte (nonché tutte quelle ulteriormente deducibili sfruttando la linea di ragionamento seguita in questa sede) sono perfettamente adatte agli accordi di quarta specie tonicizzati, indipendentemente dal posizionamento della tonicizzazione.

Se consideriamo, ad esempio, la frase nel Pentagramma (2), realizziamo immediatamente come essa possa essere serenamente adoperata in presenza delle seguenti progressioni: [Cmaj7 | G7 | Cmaj7], [Cmaj7 | D-7 G7 | Cmaj7], [Cmaj7 A-7| D-7 G7| Cmaj7].

Si considerino, pertanto, i tre seguenti Pentagrammi:

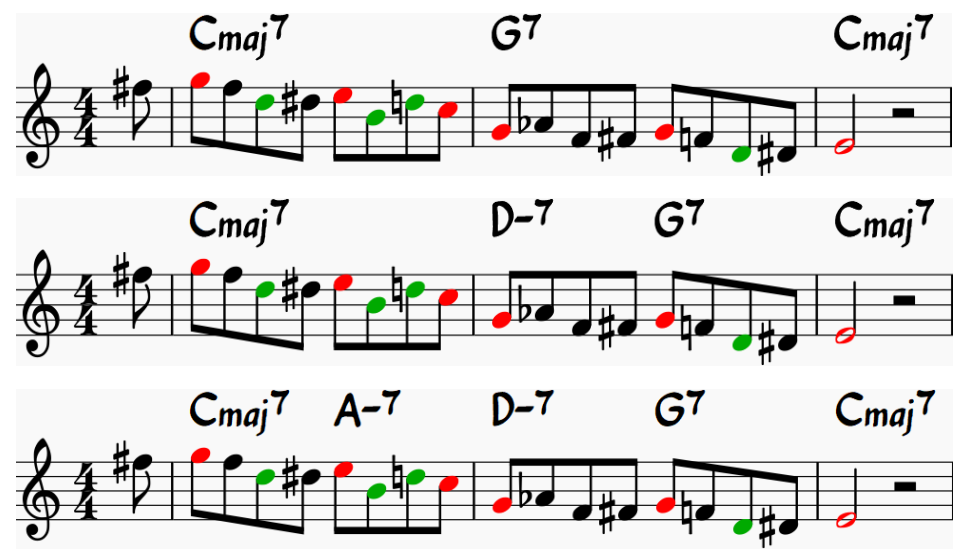

Similmente, a titolo d'ulteriore esempio, è agevole verificare come anche la frase proposta nel Pentagramma (33) sia perfettamente adatta alle progressioni armoniche appena considerate:

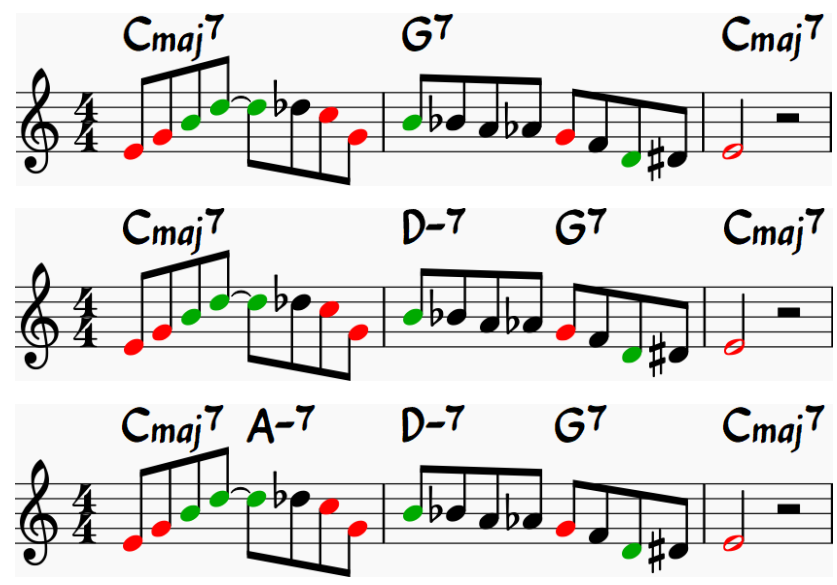

Per amore di sintesi, l'efficacia delle frasi dedotte qualora vengano adottate sostituzioni armoniche, come quelle per dominante secondaria, non è affrontata in questa sede.

\subsection{Osservazioni Finali}

Come per il precedente, tutte le frasi dedotte in questo capitolo, al netto di possibili "arricchimenti" (si veda il Capitolo 6) e combinazioni con "pattern di pubblico dominio" [4] [25], possono essere agevolmente rinvenute esaminando le trascrizioni di alcuni assoli notevoli. [21] [22] [23] [28] 
Terza Sezione

\section{Comprendere un Brano: Cenni all'Analisi Armonica}




\section{Introduzione alla Terza Sezione}

La Terza Sezione, costituita da soli due capitoli, desidera fornire un supporto a quanti desiderino intraprendere uno studio più approfondito dei brani sui quali si desideri improvvisare. Oltre a "suonare sugli accordi", infatti, è di certo auspicabile comprendere come si possa essere pervenuti ad una particolare progressione armonica, in tal modo acquisendo, assai evidentemente, la capacità di operare sostituzioni coerenti ed efficaci in funzione delle proprie esigenze improvvisative.

Nel Nono Capitolo vengono brevemente richiamate le principali sostituzioni armoniche (diatoniche, per dominanti secondarie, di tritono, per espansione ovvero completamente, alla quinta specie).

Nel Decimo Capitolo sono accuratamente esaminate le strutture armoniche (in forma parziale ovvero totale) di alcune composizioni della tradizione jazzistica d'innegabile interesse, semplificandole notevolmente a mezzo delle sostituzioni di cui sopra.

La notazione adoperata, assai inconsueta, strizza evidentemente l'occhio ad ambiti non prettamente musicali: tale scelta deriva dalla possibilità, altrove esplorata dall'autore e in questa sede non discussa, di dar vita ad una vera e propria "algebra musicale", il cui fine consisterebbe nell'effettuare una trattazione rigorosa e formale dell'armonia nella sua interezza, senz'alcun bisogno, tuttavia, di sconfinare nella fisica acustica (cui, ad ogni modo, la musica è innegabilmente connessa). 

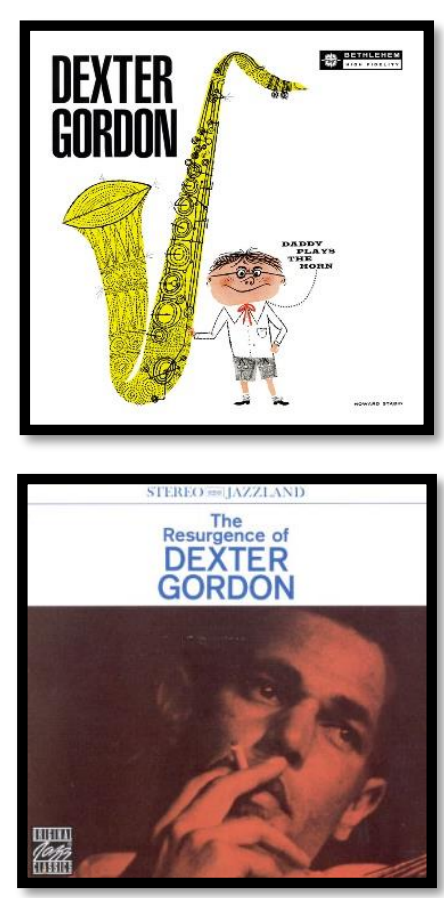

Daddy Plays the Horn

(Bethlehem, 1955)

Dexter Gordon - sassofono tenore

Kenny Drew - pianoforte

Leroy Vinnegar - contrabbasso

Lawrence Marable - batteria

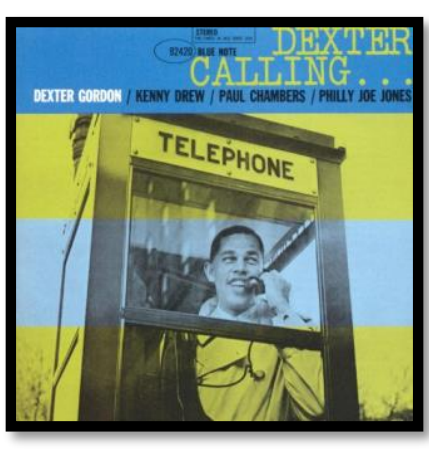

\section{The Resurgence of Dexter Gordon}

(Jazzland, 1960)

Martin Banks - tromba

Dexter Gordon - sassofono tenore

Richard Boone - trombone

Dolo Coker - pianoforte

Charles Green - contrabbasso

Lawrence Marable - batteria

\section{Dexter Calling...}

(Blue Note, 1962)

Dexter Gordon - sassofono tenore

Kenny Drew - pianoforte

Paul Chambers - contrabbasso

Philly Joe Jones - batteria

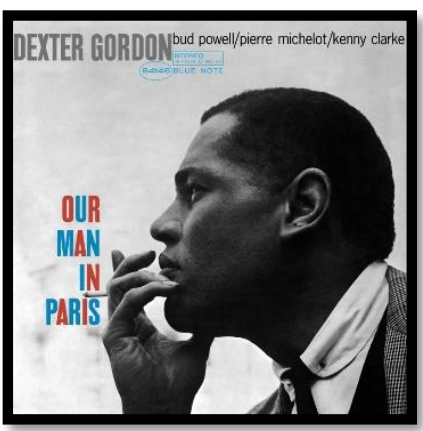

\section{Our Man in Paris}

(Blue Note, 1963)

Dexter Gordon - sassofono tenore

Bud Powell - pianoforte

Pierre Michelot - contrabbasso

Kenny Clarke - batteria

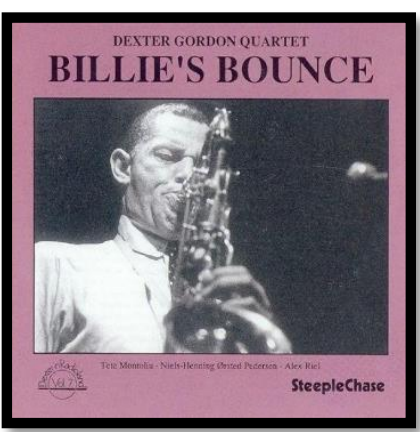

\section{Billie's Bounce}

(SteepleChase, 1979)

Dexter Gordon - sassofono tenore

Tete Montoliu - pianoforte

Niels-Henning Ørsted Pedersen - contrabbasso

Alex Riel - batteria 


\section{Sostituzioni Armoniche Fondamentali}

\subsection{Sostituzioni Diatoniche}

Due accordi scaturiti dall'armonizzazione della stessa scala sono intercambiabili se la distanza tra di essi (tra le toniche) è pari ad una terza diatonica (ascendente ovvero discendente). [7] [8] [9] [32] Riferendosi in via esclusiva all'Armonizzazione Ionica, le Sostituzioni Diatoniche nella loro interezza possono essere riassunte a mezzo delle seguenti relazioni:

$$
\begin{aligned}
& \operatorname{VIm} 7 \stackrel{\text { diat. }}{\longleftrightarrow} \operatorname{Imaj} 7 \stackrel{\text { diat. }}{\longleftrightarrow} \operatorname{IIIm} 7
\end{aligned}
$$

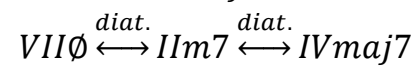

$$
\begin{aligned}
& \operatorname{Imaj} \stackrel{\text { diat. }}{\longleftrightarrow I I I m} 7 \stackrel{\text { diat. }}{\longleftrightarrow} V 7
\end{aligned}
$$

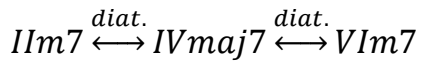

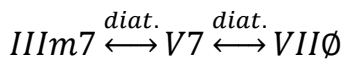

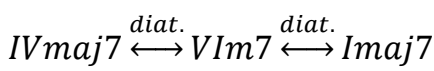

$$
\begin{aligned}
& \text { V7 } \stackrel{\text { diat. }}{\longleftrightarrow} \text { VII } \stackrel{\text { diat. }}{\longleftrightarrow} \operatorname{IIm} 7
\end{aligned}
$$

Ponendo, ad esempio, $I=C$, dalle precedenti relazioni otteniamo le seguenti:

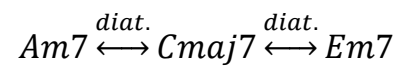

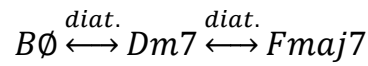

$$
\begin{aligned}
& \text { Cmaj7 } \stackrel{\text { diat. }}{\longleftrightarrow} E m 7 \stackrel{\text { diat. }}{\longleftrightarrow G 7}
\end{aligned}
$$

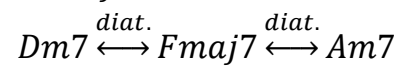

$$
\begin{aligned}
& E m 7 \stackrel{\text { diat. }}{\longleftrightarrow} G 7 \stackrel{\text { diat. }}{\longleftrightarrow} B \emptyset
\end{aligned}
$$

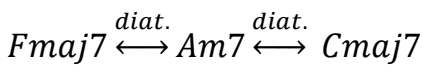

$$
\begin{aligned}
& G 7 \stackrel{\text { diat. }}{\longleftrightarrow} B \emptyset \stackrel{\text { diat. }}{\longleftrightarrow} \text { Dm7 }
\end{aligned}
$$

Le Sostituzioni Diatoniche possono essere descritte ricorrendo ad una matrice di accordi (che rappresenta ciò che potremmo denominare "Tensore Diatonico"):

$$
\operatorname{Dia}^{I o n}(I)=\left[\begin{array}{ccccccc}
I m a j 7 & 0 & I I I m 7 & 0 & 0 & V I m 7 & 0 \\
0 & I I m 7 & 0 & I V m a j 7 & 0 & 0 & V I I \varnothing \\
\operatorname{Imaj7} & 0 & I I I m 7 & 0 & V 7 & 0 & 0 \\
0 & I I m 7 & 0 & I V m a j 7 & 0 & V I m 7 & 0 \\
0 & 0 & I I I m 7 & 0 & V 7 & 0 & V I I \varnothing \\
\operatorname{Imaj} 7 & 0 & 0 & I V m a j 7 & 0 & V I m 7 & 0 \\
0 & I I m 7 & 0 & 0 & V 7 & 0 & V I I \varnothing
\end{array}\right]
$$


Una volta costruita la matrice in (15), le Sostituzioni Diatoniche possono essere espresse, con ovvio significato della notazione, nel seguente modo:

$$
D i a_{i j}^{I o n} \leftrightarrow D i a_{j i}^{I o n}
$$

Dalla (15), ponendo, ancora una volta, $I=C$, otteniamo immediatamente:

$$
\operatorname{Dia}^{\text {Ion }}(C)=\left[\begin{array}{ccccccc}
\text { Cmaj7 } & 0 & E m 7 & 0 & 0 & A m 7 & 0 \\
0 & D m 7 & 0 & F m a j 7 & 0 & 0 & B \emptyset \\
\text { Cmaj7 } & 0 & E m 7 & 0 & G 7 & 0 & 0 \\
0 & D m 7 & 0 & F m a j 7 & 0 & A m 7 & 0 \\
0 & 0 & E m 7 & 0 & G 7 & 0 & B \emptyset \\
\text { Cmaj7 } & 0 & 0 & F m a j 7 & 0 & A m 7 & 0 \\
0 & D m 7 & 0 & 0 & G 7 & 0 & B \emptyset
\end{array}\right]
$$

La Figura 1 offre un’utile rappresentazione grafica delle Sostituzioni Diatoniche.

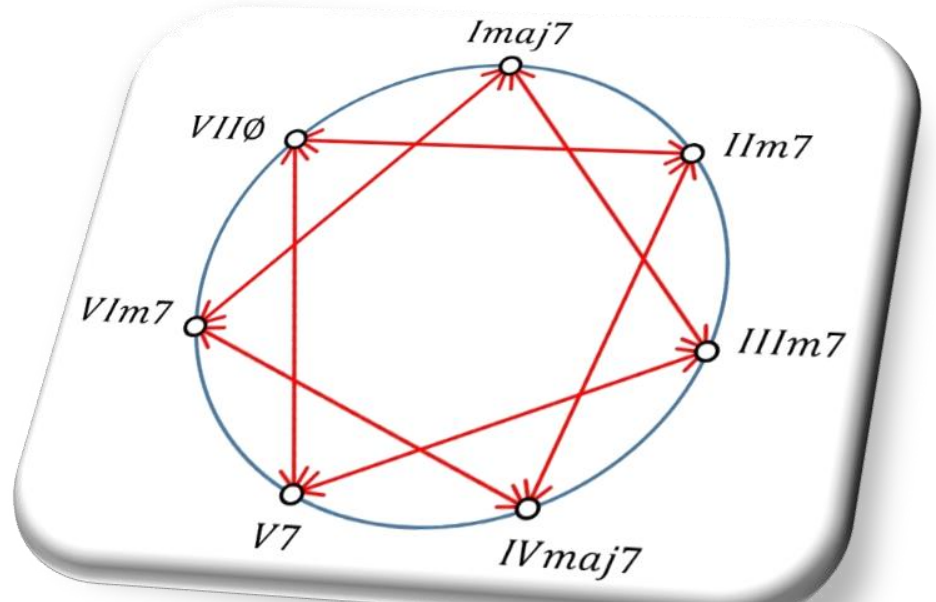

Figura 1. Sostituzioni Diatoniche

\subsection{Sostituzioni per Dominanti Secondarie}

Ogni accordo, di qualsivoglia specie ed anche qualora scaturito da una precedente sostituzione, può essere convertito in un Accordo di Settima di Dominante (Prima Specie). [7] [8] [9] [32] [33] Riferendosi, ancora una volta, agli accordi ottenuti dall'Armonizzazione Ionica, abbiamo:

$$
\begin{gathered}
I m a j 7 \stackrel{\text { dom. sec. }}{\longrightarrow} I 7=V(I V) \\
I I m 7 \stackrel{\text { dom. sec. }}{\longrightarrow} I I 7=V(V) \\
I I I m 7 \stackrel{\text { dom. sec. }}{\longrightarrow} I I I 7=V(V I) \\
I V m a j 7 \stackrel{\text { dom. sec. }}{\longrightarrow} I V 7=V(b V I I)
\end{gathered}
$$




$$
\begin{gathered}
V 7=V(I)=\text { Dominante Primaria } \\
V I m 7 \stackrel{\text { dom. sec. }}{\longrightarrow} V I 7=V(I I) \\
V I I \emptyset \stackrel{\text { dom. sec. }}{\longrightarrow} V I I 7=V(I I I)
\end{gathered}
$$

Ponendo, ad esempio, $I=C$, dalle precedenti relazioni otteniamo:

$$
\begin{gathered}
\text { Cmaj7 } \stackrel{\text { dom. sec. }}{\longrightarrow} C 7=V(F) \\
\text { Dm7 } \stackrel{\text { dom. sec. }}{\longrightarrow} D 7=V(G) \\
\text { Em7 } \stackrel{\text { dom. sec. }}{\longrightarrow} E 7=V(A) \\
F m a j 7 \stackrel{\text { dom. sec. }}{\longrightarrow} F 7=V\left(B^{b}\right) \\
G 7=V(C)=\text { Dominante Primaria } \\
\text { Am7 } \stackrel{\stackrel{\text { dom. sec. }}{\longrightarrow}}{\longrightarrow} A 7=V(D) \\
B \emptyset \stackrel{\text { dom. sec. }}{\longrightarrow} B 7=V(E)
\end{gathered}
$$

Dalla (28) deduciamo che l'accordo di Prima Specie ricavato sul quarto grado (F7, nel caso specifico) risolve "autenticamente" su una tonalità ( $B^{b}$, nel caso specifico) non direttamente riconducibile al Modo Ionico di $C$ (siccome $B^{b}$ non appartiene alla Ionica di $C$ ). Per tale motivo, la Dominante Secondaria derivata dal quarto grado viene comunemente denominata "non funzionale".

\subsection{Sostituzioni di Tritono}

Ogni Accordo di Prima Specie, soprattutto se alterato ed anche qualora scaturito da una precedente sostituzione, può essere rimpiazzato da un accordo della stessa specie distante tre toni. [7] [8] [9] [32] [33] [34] Indicando con $X$ una generica nota della Scala Cromatica, e con $t$ l'intervallo di un tono, abbiamo:

$$
\begin{aligned}
& X 7 \stackrel{\text { trit. }}{\longrightarrow} Y 7 \\
& Y=X+3 t
\end{aligned}
$$

Ponendo, ad esempio, $I=C$, da (32) e (33) ricaviamo:

$$
\text { C7 } \stackrel{\text { trit. }}{\longrightarrow} G^{b} 7 \equiv F^{\#} 7
$$

\subsection{Sostituzioni alla Quinta Specie}

Ogni Accordo di Settima di Dominante, in specie se provvisto della nona bemolle ed anche qualora scaturito da una precedente sostituzione, può essere rimpiazzato da un Accordo Diminuito (Quinta Specie) distante, ascendendo, una terza maggiore, una quinta giusta, una settima minore, una nona bemolle. [7] [8] [9] [32] [33] [34] In altri termini, adoperando una notazione assai coincisa, abbiamo:

$$
\begin{gathered}
X 7^{(b 9)} \stackrel{\text { dim. }}{\longrightarrow} Y \operatorname{dim} 7 \\
Y=X+2 t+\frac{3 n}{2} t \quad n=0,1,2,3
\end{gathered}
$$


Più esplicitamente, possiamo scrivere:

$$
X 7^{(b 9)} \stackrel{\text { dim. }}{\longrightarrow}\left\{\begin{array}{lc}
\langle X+2 t\rangle \operatorname{dim} 7 & n=0, \text { terza maggiore } \\
\left\langle X+\frac{7}{2} t\right\rangle \operatorname{dim} 7 & n=1, \text { quinta giusta } \\
\langle X+5 t\rangle \operatorname{dim} 7 & n=2, \text { settima minore } \\
\left\langle X+\frac{13}{2} t\right\rangle \operatorname{dim} 7 & n=3, \text { nona bemolle }
\end{array}\right.
$$

Ponendo ancora una volta, sempre a titolo d'esempio, $X=C$, dalla (37) otteniamo:

$$
C 7^{(b 9)} \stackrel{\operatorname{dim} .}{\longrightarrow} \begin{cases}\text { Edim } 7 & n=0, \text { terza maggiore } \\ \text { Gdim } 7 & n=1, \text { quinta giusta } \\ B^{b} \operatorname{dim} 7 & n=2, \text { settima minore } \\ D^{b} \operatorname{dim} 7 & n=3, \text { nona bemolle }\end{cases}
$$

\subsection{Sostituzioni per Espansione (Completamento)}

Com'è noto, gli Accordi di Dominante 9sus4 e b9sus4 possono essere espressi ricorrendo ai cosiddetti Slash Chord. In termini assai formali, con ovvio significato della notazione, possiamo scrivere:

$$
\begin{aligned}
V 7^{9 \text { sus } 4}(X) & =\frac{\operatorname{Im} 7(X)}{\left\langle X+\frac{7}{2} t\right\rangle} \\
V 7^{\text {b9sus } 4}(X) & =\frac{\operatorname{IIm} 7 b 5(X)}{\left\langle X+\frac{7}{2} t\right\rangle}
\end{aligned}
$$

In virtù di (39) e (40), possiamo affermare che, almeno in certa misura, ogni Accordo di Settima di Dominante può essere immaginato come preceduto (e pertanto pù̀ essere completato) da un Accordo di Seconda ovvero Terza Specie (di Minore Settima ovvero Semi-Diminuito) distante una quarta giusta discendente. [2] [8] [9] Di conseguenza, impiegando una linea verticale per separare due battute ovvero movimenti consecutivi, abbiamo:

$$
V 7 \stackrel{\text { esp. }}{\longrightarrow}\left\{\begin{array}{l}
\operatorname{IIm} 7 \quad \mid V 7 \\
\operatorname{IIm} 7 b 5 \mid V 7
\end{array}\right.
$$

Ponendo, per esempio, $X=C$, da (39) e (40) otteniamo:

$$
\begin{aligned}
& G 7^{9 s u s 4}=\frac{D m 7}{G} \\
& G 7^{\text {b9sus } 4}=\frac{D \emptyset}{G}
\end{aligned}
$$

Conseguentemente, coerentemente con la posizione sopra effettuata $(X=C)$, dalla (41) abbiamo:

$$
G 7 \stackrel{\text { esp. }}{\longrightarrow}\left\{\begin{array}{l}
D m 7 \mid G 7 \\
D \varnothing \mid G 7
\end{array}\right.
$$




\subsection{Rhythm Changes (Bridge)}

Consideriamo la seguente banale progressione armonica:

\begin{tabular}{|l|l|l|l|}
\hline Imaj7 & $\operatorname{Imaj7}$ & $V \operatorname{Im} 7$ & $V m 7$ \\
\hline $\operatorname{IIm} 7$ & $\operatorname{IIm} 7$ & $V 7$ & $V 7$ \\
\hline
\end{tabular}

Progressione Armonica 1.1

Ponendo $I=B^{b}$, otteniamo immediatamente:

\begin{tabular}{|l|l|l|l|}
\hline B $^{\mathrm{b}}$ maj7 & B $^{\mathrm{b} m a j 7}$ & Gm7 & Gm7 \\
\hline $\mathrm{Cm} 7$ & $\mathrm{Cm} 7$ & F7 & F7 \\
\hline
\end{tabular}

\section{Progressione Armonica 1.2}

Tenendo conto della precedente progressione, effettuiamo le seguenti sostituzioni armoniche:

$$
\begin{gathered}
\text { batt. 1,2: } B^{b} \text { maj7 } \stackrel{\text { diat. }}{\longrightarrow} \text { Dm7 } \stackrel{\text { dom. sec. }}{\longrightarrow} D 7 \\
\text { batt. 3,4: Gm7 } \stackrel{\text { dom. sec. }}{\longrightarrow} G 7 \\
\text { batt.5,6: Cm7 } \stackrel{\text { dom. sec. }}{\longrightarrow} C 7
\end{gathered}
$$

Dalla Progressione Armonica 1.2, in virtù di (45), (46) e (47), finalmente otteniamo:

\begin{tabular}{|l|l|l|l|}
\hline D7 & D7 & G7 & G7 \\
\hline C7 & C7 & F7 & F7 \\
\hline
\end{tabular}

Progressione Armonica 1.3

\subsection{I'm Getting Sentimental Over You (prime 8 battute)}

Consideriamo ora la seguente progressione armonica:

\begin{tabular}{|l|l|ll|ll|}
\hline Imaj7 & $V 7$ & Imaj7 & IVm7 \\
\hline $\operatorname{IIm} 7$ & $V 7$ & Imaj7 VIm7 & IIm7 & \\
& & & & \\
\hline
\end{tabular}

Progressione Armonica 2.1 
Ponendo $I=F$, otteniamo immediatamente:

\begin{tabular}{|l|l|ll|ll|}
\hline Fmaj7 & C7 & Fmaj7 & Dm7 & \\
\hline Gm7 & C7 & Fmaj7 Dm7 & Gm7 C7 \\
\hline
\end{tabular}

Progressione Armonica 2.2

Tenendo conto della precedente progressione, effettuiamo le seguenti sostituzioni armoniche:

$$
\begin{aligned}
\text { batt. 2: } C 7 \stackrel{\text { diat. }}{\longrightarrow} E \varnothing \stackrel{\text { dom. sec. }}{\longrightarrow} E 7 \stackrel{\text { esp. }}{\longrightarrow} B m 7 \mid E 7 \\
\text { batt. 3: Fmaj7 } \stackrel{\text { dom. sec. }}{\longrightarrow} F 7=V\left(B^{b}\right) \stackrel{\text { diat. }}{\longrightarrow} A \emptyset \\
\text { batt. 4,6:Dm7 } \stackrel{\text { dom. sec. }}{\longrightarrow} D 7 \\
\text { batt. 5,8: Gm7 } \stackrel{\text { dom. sec. }}{\longrightarrow} G 7
\end{aligned}
$$

Dalla Progressione Armonica 2.2, in virtù di (48), (49), (50) e (51), otteniamo immediatamente:

\begin{tabular}{|l|ll|ll|ll|}
\hline Fmaj7 & Bm7 & E7 & Am7b5 & D7 & \\
\hline G7 & C7 & Fmaj7 D7 & Gm7 & C7 \\
\hline
\end{tabular}

\section{Progressione Armonica 2.3}

Vale la pena precisare come, in taluni casi, la procedura finalizzata all'ottenimento d'un particolare accordo (nonché, al limite, d'una progressione armonica nella sua interezza) non sia unica. Tanto per fornire un esempio, è agevole verificare come l'accordo nella terza battuta della Progressione Armonica 2.3 possa essere alternativamente (e più correttamente) dedotto ricorrendo alle seguenti banali sostituzioni:

$$
\begin{aligned}
& \text { batt. 3: Fmaj7 } \stackrel{\text { diat. }}{\longrightarrow} D m 7 \\
& \text { batt. 4: Dm7 } \stackrel{\text { dom. sec. }}{\longrightarrow} D 7 \\
& \text { batt. 3,4:D7 } \stackrel{\text { esp. }}{\longrightarrow} A \emptyset \mid D 7
\end{aligned}
$$

\subsection{Stella by Starlight (prime 8 battute)}

Consideriamo la seguente progressione:

\begin{tabular}{|l|l|l|l|}
\hline $\operatorname{IIm} 7$ & $\operatorname{IIm} 7$ & $V 7$ & $V 7$ \\
\hline $\operatorname{Imaj7}$ & $\operatorname{Imaj7}$ & $\operatorname{IIm} 7$ & $V 7$ \\
\hline
\end{tabular}

Progressione Armonica 3.1

Ponendo $I=B^{b}$, otteniamo: 


\begin{tabular}{|l|l|l|l|}
\hline $\mathrm{Cm} 7$ & $\mathrm{Cm} 7$ & $\mathrm{~F} 7$ & F7 \\
\hline B $^{\mathrm{b} m a j 7}$ & B $^{\mathrm{b} \text { maj7 }}$ & $\mathrm{Cm} 7$ & F7 \\
\hline
\end{tabular}

Progressione Armonica 3.2

Sulla base della precedente progressione, effettuiamo le seguenti sostituzioni:

$$
\begin{aligned}
\text { batt. 1,2: } C m 7 \stackrel{\text { diat. }}{\longrightarrow} A \emptyset \stackrel{\text { dom. sec. }}{\longrightarrow} A 7 \stackrel{\text { esp. }}{\longrightarrow} E \emptyset \mid A 7 \\
\text { batt. 3,4: } F 7 \stackrel{\text { esp. }}{\longrightarrow} C m 7 \mid F 7 \\
\text { batt.5,6: } B^{b} \text { maj7 } \stackrel{\text { dom. sec. }}{\longrightarrow} B^{b} 7 \stackrel{\text { esp. }}{\longrightarrow} F m 7 \mid B^{b} 7 \\
\text { batt. 7: Cm7 } \stackrel{\text { diat. }}{\longrightarrow} E^{b} \text { maj7 } \\
\text { batt. 8: } F 7 \stackrel{\text { diat. }}{\longrightarrow} D m 7 \stackrel{\text { dom. sec. }}{\longrightarrow} D 7 \stackrel{\text { trit. }}{\longrightarrow} A^{b} 7
\end{aligned}
$$

Dalla Progressione Armonica 3.2, sfruttando (55), (56), (57), (58) e (59), finalmente abbiamo:

\begin{tabular}{|l|l|l|l|}
\hline Em7 & A7 & Cm7 & F7 \\
\hline Fm7 & $\mathrm{B}^{\mathrm{b} 7}$ & $\mathrm{E}^{\mathrm{b}} \mathrm{maj} 7$ & $\mathrm{~A}^{\mathrm{b}} 7$ \\
\hline
\end{tabular}

\section{Progressione Armonica 3.3}

$A^{b} 7$ risolve su $B^{b}$ maj7. La progressione ${ }^{b}$ VII7 | Imaj7 è denominata "Back-Door Solution". [8] [9]

\subsection{Easy Living (prime 8 battute)}

Consideriamo la seguente progressione (assai banale e ripetitiva):

\begin{tabular}{|ll|ll|ll|ll|}
\hline Imaj7 & $V I m 7$ & $\operatorname{IIm} 7$ & $V 7$ & $\operatorname{Imaj7}$ & $V I m 7$ & $\operatorname{IIm} 7$ & $V 7$ \\
\hline $\operatorname{Imaj7}$ & $V \operatorname{Im} 7$ & $\operatorname{IIm} 7$ & $V 7$ & $\operatorname{Imaj7}$ & $V I m 7$ & $\operatorname{IIm} 7$ & $V 7$ \\
& & & & & & & \\
\hline
\end{tabular}

\section{Progressione Armonica 4.1}

Ponendo $I=A^{b}$, otteniamo immediatamente:

\begin{tabular}{|ll|ll|ll|ll|}
\hline $\mathrm{A}^{\mathrm{b}}$ maj7 & Fm7 & $\mathrm{B}^{\mathrm{b}} \mathrm{m} 7$ & $\mathrm{E}^{\mathrm{b}} 7$ & $\mathrm{~A}^{\mathrm{b} m a j} 7$ & Fm7 & $\mathrm{B}^{\mathrm{b} m} 7$ & $\mathrm{E}^{\mathrm{b}} 7$ \\
\hline $\mathrm{A}^{\mathrm{b}}$ maj7 & Fm7 & $\mathrm{B}^{\mathrm{b} m} 7$ & $\mathrm{E}^{\mathrm{b}} 7$ & $\mathrm{~A}^{\mathrm{b}} \mathrm{maj} 7$ & Fm7 & $\mathrm{B}^{\mathrm{b}} \mathrm{m} 7$ & $\mathrm{E}^{\mathrm{b}} 7$ \\
\hline
\end{tabular}

Progressione Armonica 4.2

Tenendo conto della precedente progressione, effettuiamo le seguenti sostituzioni:

$$
\text { batt.1: } F m 7 \stackrel{\text { dom. sec. }}{\longrightarrow} F 7 \stackrel{\text { dim. }}{\longrightarrow} \operatorname{Adim} 7
$$




$$
\begin{array}{r}
\text { batt. 2: } E^{b} 7 \stackrel{\text { diat. }}{\longrightarrow} G \emptyset \stackrel{\text { dom. sec. }}{\longrightarrow} G 7 \stackrel{\text { dim. }}{\longrightarrow} \text { Bdim7 } \\
\text { batt. 3,6: } A^{b} \text { maj7 } \stackrel{\text { diat. }}{\longrightarrow} \text { Cm } 7 \\
\text { batt. 3: Fm7 } \stackrel{\text { diat. }}{\longrightarrow} A^{b} \text { maj7 } \stackrel{\text { dom. sec. }}{\longrightarrow} A^{b} 7 \stackrel{\text { esp. }}{\longrightarrow} E^{b} m 7 \mid A^{b} 7 \\
\text { batt. } 4: B^{b} m 7 \stackrel{\text { diat. }}{\longrightarrow} D^{b} \text { maj7 } \\
\text { batt. } 4: E^{b} 7 \stackrel{\text { diat. }}{\longrightarrow} C m 7 \stackrel{\text { dom. sec. }}{\longrightarrow} C 7 \stackrel{\text { trit. }}{\longrightarrow} G^{b} 7 \stackrel{\text { esp. }}{\longrightarrow} D^{b} m 7 \mid G^{b} 7 \\
\text { batt. } 7: \text { Fm7 } \stackrel{\text { dom. sec. }}{\longrightarrow} F 7
\end{array}
$$

Dalla Progressione Armonica 4.2, in virtù di (60), (61), (62), (63), (64), (65) e (66), otteniamo:

\begin{tabular}{|ll|ll|lll|lll|}
\hline $\mathrm{A}^{\mathrm{b}}$ maj7 & Ao7 & $\mathrm{B}^{\mathrm{b} m} 7$ & $\mathrm{Bo} 7$ & $\mathrm{Cm} 7$ & $\mathrm{E}^{\mathrm{b} m} 7$ & $\mathrm{~A}^{\mathrm{b}} 7$ & $\mathrm{D}^{\mathrm{b}}$ maj7 & $\mathrm{D}^{\mathrm{b}} \mathrm{m} 7$ & $\mathrm{G}^{\mathrm{b}} 7$ \\
\hline $\mathrm{A}^{\mathrm{b}}$ maj7 & Fm7 & $\mathrm{B}^{\mathrm{b} m} 7$ & $\mathrm{E}^{\mathrm{b}} 7$ & $\mathrm{Cm} 7$ & $\mathrm{~F} 7$ & & $\mathrm{~B}^{\mathrm{b}} \mathrm{m} 7$ & $\mathrm{E}^{\mathrm{b}} 7$ & \\
\hline
\end{tabular}

\section{Progressione Armonica 4.3}

L'ultimo accordo in quarta battuta $\left(G^{b} 7\right)$ risolve su $A^{b}$ maj7: abbiamo ottenuto, ancora una volta, una “Back-Door Solution” (in tal caso completa, siccome $G^{b} 7$ è preceduto da $D^{b} m 7$ ).

\begin{tabular}{|c|c|c|c|c|c|}
\hline Bmaj7 & $\mathrm{G}^{\#} \mathrm{~m} 7$ & $\mathrm{C}^{\#} \mathrm{~m} 7$ & $\mathrm{~F}^{\# 7}$ & Bmaj7 & $\mathrm{G}^{\#} \mathrm{~m} 7$ \\
\hline $\mathrm{C}^{\#} \mathrm{~m} 7$ & $\mathrm{~F}^{\#} 7$ & Bmaj7 & $\mathrm{F}^{\#} 7$ & Bmaj7 & $\mathrm{F}^{\#} 7$ \\
\hline Bmaj7 & & $\mathrm{G}^{\#} \mathrm{~m} 7$ & & $\mathrm{C}^{\#} \mathrm{~m} 7$ & $\mathrm{~F}^{\#} 7$ \\
\hline Bmaj7 & & $\mathrm{F}^{\#} 7$ & & Bmaj7 & $\mathrm{F}^{\#} 7$ \\
\hline
\end{tabular}

\subsection{Giant Steps}

Consideriamo adesso la seguente progressione armonica:

\begin{tabular}{|ll|ll|l|l|}
\hline Imaj7 $\quad$ VIm7 & IIm7 & V7 & Imaj7 & VIm7 \\
\hline IIm7 & V7 & Imaj7 & V7 & Imaj7 & $V 7$ \\
\hline Imaj7 & VIm7 & IIm7 & $V 7$ \\
\hline Imaj7 & $V 7$ & Imaj7 & $V 7$ \\
\hline
\end{tabular}

Progressione Armonica 5.1

Ponendo $I=B$, otteniamo agevolmente:

Progressione Armonica 5.2

Sulla base della progressione precedente, effettuiamo le seguenti sostituzioni: 


$$
\begin{gathered}
\text { batt. } 1: G^{\#} m 7 \stackrel{\text { dom. sec. }}{\longrightarrow} G^{\#} 7 \equiv A^{b} 7 \stackrel{\text { trit. }}{\longrightarrow} D 7 \\
\text { batt. 2,5,11: } C^{\#} m 7 \stackrel{\text { dom. sec. }}{\longrightarrow} C^{\#} 7 \equiv D^{b} 7 \stackrel{\text { trit. }}{\longrightarrow} G 7 \\
\text { batt. 2,5: } F^{\#} 7 \stackrel{\text { diat. }}{\longrightarrow} A^{\#} \emptyset \stackrel{\text { dom. sec. }}{\longrightarrow} A^{\#} 7 \equiv B^{b} 7 \\
\text { batt.3,6,9,15: Bmaj7 } \stackrel{\text { diat. }}{\longrightarrow} D^{\#} m 7 \stackrel{\text { dom. sec. }}{\longrightarrow} D^{\#} 7 \equiv E^{b} 7 \\
\text { batt. 4,10: } G^{\#} m 7 \stackrel{\text { dom. sec. }}{\longrightarrow} G^{\#} 7 \equiv A^{b} 7 \stackrel{\text { trit. }}{\longrightarrow} D 7 \stackrel{\text { esp. }}{\longrightarrow} A m 7 \mid D 7 \\
\text { batt. 8,14: } F^{\#} 7 \stackrel{\text { diat. }}{\longrightarrow} A^{\#} \phi \stackrel{\text { dom. sec. }}{\longrightarrow} A^{\#} 7 \equiv B^{b} 7 \stackrel{\text { esp. }}{\longrightarrow} F m 7 \mid B^{b} 7 \\
\text { batt. } 12,16: F^{\#} 7 \stackrel{\text { esp. }}{\longrightarrow} C^{\#} m 7 \mid F^{\#} 7
\end{gathered}
$$

\begin{tabular}{|c|c|c|c|c|c|c|}
\hline Bmaj7 & D7 & G7 & $\mathrm{B}^{\mathrm{b}} 7$ & $\mathrm{E}^{\mathrm{b} 7}$ & Am7 & D7 \\
\hline G7 & $\mathrm{B}^{\mathrm{b} 7}$ & $\mathrm{E}^{\mathrm{b}} 7$ & $\mathrm{~F}^{\# 7}$ & Bmaj7 & Fm7 & $\mathrm{B}^{\mathrm{b}} 7$ \\
\hline $\mathrm{E}^{\mathrm{b} 7}$ & & Am7 & D7 & G7 & $\mathrm{C}^{\#} \mathrm{~m} 7$ & $\mathrm{~F} \# 7$ \\
\hline Bmaj7 & & Fm7 & $\mathrm{B}^{\mathrm{b}} 7$ & $\mathrm{E}^{\mathrm{b} 7}$ & $\mathrm{C}^{\#} \mathrm{~m} 7$ & F\#7 \\
\hline
\end{tabular}

Dalla Progressione Armonica 5.2, in virtù di (67), (68), (69), (70), (71), (72) e (73), ricaviamo:

Progressione Armonica 5.3

La Progressione Armonica 5.3 non rappresenta ancora il risultato desiderato. Al fine d'ottenere la progressione originale di Coltrane, occorre modificare ulteriormente gli accordi riportati in rosso. A tal proposito, introduciamo una banale sostituzione armonica, spesso denominata semplicemente " $d i$ Qualità " [35] [36], che consente di convertire istantaneamente un Accordo di Prima Specie in uno di Quarta, evitando il ricorso al cosiddetto "Scambio Parallelo". A legittimazione della procedura, basti pensare che le due specie di cui sopra sono caratterizzate dalla stessa triade (dalle stesse note cordali): in altri termini, gli accordi di Prima e Quarta Specie differiscono esclusivamente nella settima (che può essere considerata, almeno in certo senso, una tensione fondamentale). Il "Turnaround di Tadd

\begin{tabular}{|c|c|c|c|c|c|c|}
\hline Bmaj7 & D7 & Gmaj7 & $\mathrm{B}^{\mathrm{b} 7}$ & $\mathrm{E}^{\mathrm{b}} \mathrm{maj} 7$ & Am7 & D7 \\
\hline Gmaj7 & $\mathrm{B}^{\mathrm{b}} 7$ & $\mathrm{E}^{\mathrm{b}} \mathrm{maj} 7$ & $\mathrm{~F} \# 7$ & Bmaj7 & Fm7 & $\mathrm{B}^{\mathrm{b} 7}$ \\
\hline $\mathrm{E}^{\mathrm{b}} \mathrm{maj} 7$ & & Am7 & D7 & Gmaj7 & $\mathrm{C}^{\#} \mathrm{~m} 7$ & $\mathrm{~F}^{\# 7}$ \\
\hline Bmaj7 & & Fm7 & $\mathrm{B}^{\mathrm{b} 7}$ & $\mathrm{E}^{\mathrm{b}} \mathrm{maj} 7$ & $\mathrm{C}^{\#} \mathrm{~m} 7$ & $\mathrm{~F}^{\# 7}$ \\
\hline
\end{tabular}
Dameron (o Coltrane) " [4] [33], tanto per fornire un esempio notevole, è ottenuto proprio ricorrendo a tale sostituzione. In definitiva, possiamo scrivere:

$$
\begin{gathered}
\text { batt. 2,5,11:G7 } \stackrel{\text { dom. /maj. }}{\longrightarrow} \text { Gmaj7 } \\
\text { batt.3,6,9,15: } E^{b} 7 \stackrel{\text { dom. /maj. }}{\longrightarrow} E^{b} \text { maj7 }
\end{gathered}
$$

Dalla Progressione Armonica 5.3, tenendo conto di (74) e (75), finalmente otteniamo: 


\subsection{Osservazioni Finali}

Iniziamo da una banale sequenza di quattro battute (o movimenti, e insiemi costituiti dallo stesso numero di movimenti) contenenti uno stesso accordo (nel nostro caso, un Accordo di Quarta Specie):

\begin{tabular}{|l|l|l|l|}
\hline Imaj7 & Imaj7 & Imaj7 & Imaj7 \\
\hline
\end{tabular}

Progressione Armonica 6.1

Sebbene non possa essere propriamente considerata una sostituzione armonica, è possibile ricorrere alla Tonicizzazione [2] [7] [8] [9] al fine d'ottenere una prima, banale "fluttuazione armonica" (nulla più che una Cadenza Autentica ovvero Perfetta):

\begin{tabular}{|l|l|l|l|}
\hline Imaj7 & Imaj7 & V7 & V7 \\
\hline
\end{tabular}

\section{Progressione Armonica 6.2}

Effettuiamo ora le seguenti sostituzioni:

$$
\begin{aligned}
& \text { batt.(mov.) 3,4:V7 } \stackrel{\text { exp. }}{\longrightarrow} \operatorname{IIm} 7 \mid V 7 \\
& \text { batt.(mov.) 2: Imaj7 } \stackrel{\text { diat. }}{\longrightarrow} \operatorname{VIm} 7
\end{aligned}
$$

Dalla Progressione Armonica 6.2, in virtù di (76) e (77), otteniamo immediatamente:

\begin{tabular}{|l|l|l|l|}
\hline Imaj7 & $\operatorname{VIm} 7$ & $\operatorname{IIm} 7$ & $V 7$ \\
\hline
\end{tabular}

\section{Progressione Armonica 6.3}

Partendo da un singolo accordo di Quarta Specie è evidentemente possibile ottenere un Turnaround Maggiore. Conseguentemente, tutte le progressioni armoniche dedotte nella sezione precedente possono essere immaginate come scaturite da un singolo accordo. In certo senso, potremmo addirittura affermare che, al netto di alcune progressioni espressamente basate sulla Cadenza Plagale "Pura” (IVmaj7| Imaj7), tutti i cosiddetti “Standard Jazz” possono essere considerati come originati da un singolo accordo (di Quarta Specie ovvero, sfruttando l'armonizzazione di qualsivoglia scala minore, di Seconda). Ci siamo riferiti alla Cadenza Plagale "Pura" siccome quella "Autentica" o "Estesa" (IVmaj7 | V7 | Imaj7) può essere immediatamente ricavata, ricorrendo a una Sostituzione Diatonica, dalla progressione IIm7 | V7 | Imaj7 che, a sua volta, può essere ottenuta da un singolo Accordo di Quarta Specie sfruttando una Tonicizzazione e, come in (76), un'Espansione. 


\section{Ringraziamenti}

Ringrazio anzitutto gli Amici e Maestri Francesco D'Errico, Giulio Martino e Sandro Deidda, per l'imprescindibile supporto offertomi, senz'alcuna soluzione di continuità, nella prosecuzione della personale formazione jazzistica e nella realizzazione del presente lavoro.

Ringrazio mia madre, Giuseppina, senza l'entusiasmo e la tenerezza della quale mai avrei trovato la forza d'intraprendere un nuovo percorso di studi, a dispetto della passione dalla quale ero animato.

Ringrazio lo staff del "Bar Capri" di Battipaglia (SA), presso il quale, da oltre quattordici anni, rivesto il ruolo di pianista stabile e direttore artistico: in particolare, ringrazio i fratelli Carmine e Antonietta Monaco, che con coraggio e determinazione continuano ad impegnarsi strenuamente nella gratuita diffusione della cultura jazzistica, in specie tra i più giovani.

Ringrazio i revisori anonimi e i membri degli staff editoriali delle riviste scientifiche internazionali presso le quali ho pubblicato, negli ultimi due anni, numerosi articoli di ricerca in ambito jazzistico che hanno notevolmente contribuito alla redazione di questo volume.

Infine, ringrazio mio padre, cui questo lavoro è dedicato, per avermi finalmente visto. 
[1] Cataldo, C., Martino, G. (2018). La Scala Dominante Be-Bop: Definizione ed Utilizzo - The Be-Bop Dominant Scale: Definition and Usage. Journal of Science, Humanities and Arts (JOSHA), 5(2). https://dx.doi.org/10.17160/josha.5.2.389

[2] Levine, M. (2009). The Jazz Theory Book (Italian Edition by F. Jegher). Milan, IT: Curci Jazz.

[3] Wise. L. (1983). Bebop Bible - The Musicians Dictionary of Melodic Lines. United States: REH Publications.

[4] Coker, J., Casale, J., \& Campbell, G. (1982). Patterns for Jazz. Los Angeles, CA: Alfred Publishing Co. Inc.

[5] Cataldo, C. (2018). A Simplified Introduction to Music Algebra: from the Scale Vectors to the Modal Tensor. International Journal of Advanced Engineering Research and Science, 5(1), 111-113. https://dx.doi.org/10.22161/ijaers.5.1.16

[6] Cataldo, C. (2018). Algebra Musicale: dai Vettori Scala al Tensore Modale - Music Algebra: from the Scale Vectors to the Modal Tensor. Journal of Science, Humanities and Arts (JOSHA), 5(1). https://dx.doi.org/10.17160/josha.5.1.383

[7] D’Errico, F. (2017). Armonia Funzionale e Modalità - Rudimenti per l'Improvvisazione a Indirizzo Jazzistico. Naples, Italy: Editoriale Scientifica.

[8] Cataldo, C. (2018). Towards a Music Algebra: Fundamental Harmonic Substitutions in Jazz. International Journal of Advanced Engineering Research and Science, 5(1), 52-57. https://dx.doi.org/10.22161/ijaers.5.1.9

[9] Cataldo, C. (2018). Jazz e Sostituzioni Armoniche: Verso un Nuovo Formalismo - Jazz and Harmonic Substitutions: Towards a New Formalism. Journal of Science, Humanities and Arts (JOSHA), 5(1). https://dx.doi.org/10.17160/josha.5.1.381

[10] Cataldo, C., Deidda, S., D’Errico, F., Martino, G. (2018). Definition and Usage of the Be-Bop Minor Scales (part 1): the Be-Bop Melodic Minor. Journal of Science, Humanities and Arts (JOSHA), 5(2). https://dx.doi.org/10.17160/josha.5.2.395

[11] Cataldo, C., Deidda, S., D’Errico, F., Martino, G. (2018). Definition and Usage of the Be-Bop Minor Scales (part 2): the Be-Bop Dorian Scale. Journal of Science, Humanities and Arts (JOSHA), 5(2). https://dx.doi.org/10.17160/josha.5.2.396

[12] Baker, D. (1988). How to Play Bebop (Volume 1). Los Angeles, CA: Alfred Publishing Co. Inc.

[13] Baker, D. (1988). How to Play Bebop (Volume 2). Los Angeles, CA: Alfred Publishing Co. Inc.

[14] Baker, D. (1988). How to Play Bebop (Volume 3). Los Angeles, CA: Alfred Publishing Co. Inc.

[15] Cataldo, C., Deidda, S., D’Errico, F., Martino, G. (2018). Definition and Usage of the Be-Bop Minor Scales (part 3): the Be-Bop Natural Minor Scale. Journal of Science, Humanities and Arts (JOSHA), 5(3). https://dx.doi.org/10.17160/josha.5.3.407

[16] Cataldo, C., Martino, G. (2018). La Scala Maggiore Be-Bop: Definizione ed Utilizzo - The Be-Bop Major Scale: Definition and Usage. Journal of Science, Humanities and Arts (JOSHA), 5(2). https://dx.doi.org/10.17160/josha.5.2.393

[17] Baker, D. (1988). Jazz Improvisation. Los Angeles, CA: Alfred Publishing Co. Inc.

[18] Cataldo, C. (2017). The Art of Improvising: the Be-Bop Language and the Dominant Seventh Chords. Art and Design Review, 5, 181-188. http://doi.org/10.4236/adr.2017.53014

[19] Cataldo, C. (2017). Il Linguaggio Be-Bop e gli Accordi di Settima di Prima Specie [The Be-Bop Language and The Dominant Seventh Chords]. Journal of Science, Humanities and Arts (JOSHA), 4(4).

[20] Garland, R. (1999). The Jazz Piano Solos of Red Garland (by Tony Genge). Houston, TX: Houston Publishing.

[21] Kelly, W. (2013). The Wynton Kelly Collection: 25 Solo Transcriptions (by Michael Miller). New Albany, IN: Jamey Aebersold Jazz.

[22] Powell, B. (1998), Bud Powell Classics (Artist Transcriptions). Milwaukee, WI: Hal · Leonard.

[23] Powell, B. (2002). The Bud Powell Collection: Piano Transcriptions (Artist Transcriptions). Milwaukee, WI: Hal -Leonard.

[24] Parker. C. (1978). Charlie Parker Omnibook. Los Angeles, CA: Atlantic Music Corporation.

[25] Nelson, O. (2010). Patterns for Improvisation. New Albany, IN Jamey Aebersold Jazz.

[26] Cataldo, C. (2017). The Art of Improvising: the Be-Bop Language and the Minor Seventh Chords. Art and Design Review, 5, 213-221. https://doi.org/10.4236/adr.2017.54017 
[27] Cataldo, C. (2017). Il Linguaggio Be-Bop e gli Accordi di Settima di Seconda Specie [The Be-Bop Language and The Minor Seventh Chords]. Journal of Science, Humanities and Arts (JOSHA), 4(4). https://dx.doi.org/10.17160/josha.4.4.339

[28] Garland, R. (1999). The Jazz Piano Solos of Red Garland (by Tony Genge). Houston, TX: Houston Publishing.

[29] D’Errico, F. (2015). Fuor di Metafora - Sette Osservazioni sull'Improvvisazione Musicale. Naples, Italy: Editoriale Scientifica.

[30] Cataldo, C. (2017). The Art of Improvising: the Be-Bop Language and the Major Seventh Chords. Art and Design Review, 5, 222-229. https://doi.org/10.4236/adr.2017.54018

[31] Cataldo, C. (2017). Il Linguaggio Be-Bop e gli Accordi di Settima di Quarta Specie [The Be-Bop Language and The Major Seventh Chords]. Journal of Science, Humanities and Arts (JOSHA), 4(4). https://dx.doi.org/10.17160/josha.4.4.341

[32] Cho, G. J. (1992). Theories and Practice of Harmonic Analysis. Lewiston, NY: E. Mellen Press.

[33] Lawn, R., Hellmer, J. (1996). Jazz: Theory and Practice. Los Angeles, CA: Alfred Pub. Co. Inc.

[34] Coker, J. (1997). Elements of the Jazz Language for the Developing Improvisor. Los Angeles, CA: Alfred Publishing Co. Inc

[35]Cataldo, C. (2018). Extreme Chord Substitutions: a Qualitative Introduction to CAT (Cataldo Advanced Transformations). Journal of Science, Humanities and Arts (JOSHA), 5(4). https://dx.doi.org/10.17160/josha.5.4.424

[36] Cataldo, C. (2018). Music Algebra: Harmonic Progressions Analysis and CAT (Cataldo Advanced Transformations). International Journal of Advanced Engineering Research and Science, 5(5), 224-227.

https://dx.doi.org/10.22161/ijaers.5.5.29 


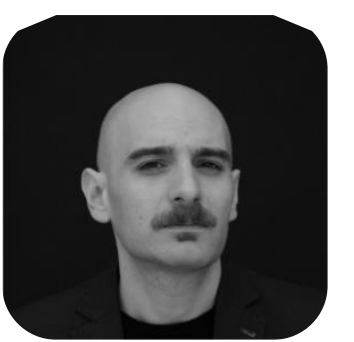

\section{Carmine Cataldo}

Independent Researcher, Battipaglia (SA), Italy

$\mathrm{PhD}$ in Mechanical Engineering

MD in Disciplines of Jazz and Improvisation - Jazz Piano

Jazz Pianist and Composer

Art Director and Resident Pianist at "Bar Capri", Battipaglia (SA), Italy

Email: catcataldo@hotmail.it

ResearchGate Profile: https://www.researchgate.net/profile/Carmine Cataldo

Carmine Cataldo was born on 13 February 1979 in Battipaglia (Salerno - Italy).

In 2004, he graduates from the University of Salerno in Mechanical Engineering; the same year, he passes the qualifying examination to the Engineers Register. In 2008, he obtains, from the University of Salerno, a PhD in Mechanical Engineering, with a final dissertation based upon the analytical modelling of the extrusion and stretching processes targeted at thermoplastic polymers.

During $\mathrm{PhD}$ research work, he mainly focuses on heat treating of steels and iron based alloys, mechanical characterization of unconventional and composite materials, innovative technologies for welded and glued joints, treatments finalized to increase the surface tension of polymeric films, the application of fuzzy logic in order to adjust the extrusion die during the film casting process and rapid prototyping by laser sintering. Currently, he is particularly interested in alternative cosmology and special relativity. His main interest lies in the attempt to preserve the validity of notions, considered as outdated, by assigning a different meaning, coherently with the phenomenological reality, to equations usually classified as relativistic. He is a member of the editorial board of the journals "Research and Reviews: Journal of Pure and Applied Physics" and "Advances in Laser Optics and Photonics".

Carmine Cataldo is also a jazz pianist and composer.

In 2018, he graduates (Master Degree, Summa Cum Laude) from the State Conservatory of Salerno in Disciplines of Jazz and Improvisation (Jazz Piano). He has been resident pianist and art director at music club "Bar Capri”, in Battipaglia (Italy), since 2004. In 2002 he ranks first in the International Jazz Competition "Baronissi Jazz Festival" (Emerging Musicians Category); moreover, he is appointed best young talent in the competition and awarded a scholarship for the Berklee Summer School at Umbria Jazz 2003. During the Berklee Clinics he studies Piano Improvisation (advanced level) with Russell Hoffmann, Ensemble Music with the guitarist Jim Kelly, Improvisation Techniques with the saxophonist Greg Badolato, Be-Bop Language with the trumpeter Jeff Stout, and attends special lectures with the renowned singer Bobby McFerrin and the legendary drummer Elvin Jones. In 2003 he ranks first in the International Jazz Competition "Baronissi Jazz Festival" (Professional Musicians Category). He has had the privilege of sharing the stage with several worldwide esteemed jazz musicians, such as Avishai Cohen (Baronissi Jazz Festival 2004), Stefano Bollani and Enrico Rava (Jazz Castello Lagopesole, 11th Edition). As a jazz pianist, he has cooperated with artists of the calibre of Alfonso Deidda, Antonio Onorato, Carla Marciano, Daniele Scannapieco, Giulio Martino, Jerry Popolo, Max Ionata, Pietro Condorelli, Sandro Deidda.

Carmine Cataldo is also a martial arts teacher.

He is appointed "Shifu" by his own master, Sifu Antonello Parisi (ITKAA), on 18 December 2016. He is a Black Belt in Shaolin Wing Chun (Superior Instructor, 4th Technical Level) and Combat Escrima Concept (Advanced Instructor, 3rd Technical Level). He has attended several seminars with renowned martial artists of the calibre of Grand Master Renè Latosa (Escrima Concepts), Sifu Maria Gröthe (Siu Lam Weng Chun), Sifu Lin Xiang Fuk (Black Flag - Hek Ki Boen Eng Chun), Master Marco Mattioni (Escrima and Wing Tsun), Master Aldo Chiari (Muay Thai Boran). 